\title{
Novos modelos estatísticos de resposta limitada para dados de compreensão leitora com estruturas dependentes
}

\author{
Sandra Elizabeth Flores Ari
}

TESE APRESENTADA

$\mathrm{AO}$

Instituto DE MATEMÁtica E EstatísticA

DA

Universidade DE SÃo PAUlo

PARA

OBTENÇÃO DO TÍTULO

$\mathrm{DE}$

DOUTOR EM CIÊNCIAS

\author{
Programa: Estatística \\ Orientador: Prof. Dr. Heleno Bolfarine \\ Coorientador: Prof. Dr. Jorge Luis Bazán Guzmán
}

São Paulo, maio de 2020 


\section{Novos modelos estatísticos de resposta limitada para dados de compreensão leitora com estruturas dependentes}

\footnotetext{
Esta versão da tese contém as correções e alterações sugeridas pela Comissão Julgadora durante a defesa da versão original do trabalho, realizada em 27/03/2020. Uma cópia da versão original está disponível no Instituto de Matemática e Estatística da Universidade de São Paulo.
}

Comissão Julgadora:

- Prof. Dr. Jorge Luis Bazán Guzmán (co-orientador) - ICMC-USP

- Prof $^{\mathrm{a}}$. Dr ${ }^{\mathrm{a}}$. Monica Sandoval Carneiro - IME-USP

- Prof. Dr. Marcos Oliveira Prates - ICEx-UFMG

- Prof. Dr. Caio Lucidius Naberezny Azevedo - IMECC-UNICAMP

- Prof. Dr. Luis Hilmar Valdivieso Serrano - DAC-PUCP 
Dedicou a presente Tese:

À meus pais, Donata e Jorge, pela vida, proteção, paciência, cuidado e formação À meu esposo Jorge, pelo amor e paciência À meus filhos, Benjamin e Paula, pelos novos aprendizagens À minhas irmãs, Dulia e Gaby, pelo apoio e carinho À meu irmão Yayo, pelo apoio e carinho 


\section{Agradecimentos}

Gostaria de expressar meu agradecimento à,

Meus pais, por permitir minha existência, pelo amor, carinho, cuidado, educação, formação, advertências, conselhos e apoio em cada etapa da minha vida o que me permitiu alcançar esta etapa. Um agradecimento especial para minha mãe quem cuidou de meus filhos como se fossem dela.

A meu esposo Jorge, pelo amor, companhia, compreensão e apoio. Pela paciência nos momentos complicados. Por ter cuidado de nossos filhos durante minha ausência.

À minhas irmãs pelo carinho, as conversas e risos que calmaram minhas preocupações.

Ao professor Heleno, por ter aceitado o convite de ser meu orientador, pelas revisões dos trabalhos iniciais da tese, as sugestões de escrita, bibliografia, temas e pela paciência.

Ao professor Jorge, por orientar meu trabalho da tese, pelos temas compartilhados, pela paciência e direcionamento da minha tese.

Ao professor Marcos, pela aprendizagem compartilhada e as mensagens que incentivaram a escrita do paper.

Aos membros da minha banca pelas observações e sugestões que ajudaram na versão final da presente tese.

Aos professores do IME, cada matéria foi um desafio, mas eles conseguiram que eu construa maior conhecimento.

Aos amigos da minha turma, pelas conversas, os espaços de aprendizagem, as palavras de alento, as visitas à bandejão.

À meus amigos peruanos no Brasil que me fizeram sentir membro de uma família. 


\section{Resumo}

Flores Ari, S.E. Novos modelos estatísticos de resposta limitada para dados de compreensão leitora com estruturas dependentes. Tese (Doutorado) - Instituto de Matemática e Estatística, Universidade de São Paulo, São Paulo, 2020.

No campo da modelagem, na aplicação prática encontramos conjuntos de dados os quais apresentam relações que dependem do instrumento de medição ou das unidades de análise localizadas no espaço as mesmas que possuem características comúns com outras unidades ao redor delas. Simultaneamente, as variáveis de resposta têm a característica de estar restrita no intervalo unitário. Ambas situações, a dependência e a restrição do soporte nas variáveis respostas, presentes na realidade, são abordadas de forma conjunta na presente tese. As relações de dependência geradas pelo instrumento de medição, como o caso das provas de Compreensão leitora são abordadas com a inclusão de efeitos aleatórios, os quais conseguem modelar relaçoes para estruturas específicas geradas pelo instrumento de medição. As relações geradas na análise de unidades com localização espacial incluem também um efeito aleatório, sui generis, com a intenção de modelar tais relações no contexto dos modelos de regressão. Ademais, o suporte unitário das variáveis são modelados considerando distribuições com suporte no intervalo $(0,1)$ como é o caso das distribuições Beta, Simplex ou Kumaraswamy. Estas propriedades específicas dos conjuntos de dados analizados tem o propósito de sugerir um modelo geral o qual abrange as relações de dependência e respostas limitadas de forma conjunta com a intenção de apresentar no presente trabalho modelos adequados com a realidade, a qual é evidenciada nos conjuntos de dados de Compreensão Leitora analizados no presente trabalho, para atingir o objetivo de obter estimativas realistas.

Palavras-chave: Compreensão leitora, resposta limitada, testlet, regressão espacial, estimação bayesiana. 


\section{Abstract}

Flores Ari, S. E. New statistical models for bounded responses in reading comprehension datasets considering related structures.

Tese (Doutorado) - Instituto de Matemática e Estatística, Universidade de São Paulo, São Paulo, 2020.

The experience with datasets suggests the existence of response variables which contain relations, as a result of applying a measure instrument or units of analysis which are localized in the space and admit similar characteristics with units around. At the same time, response variables have the typical feature of being bounded in the interval $(0,1)$. Both situations, relations of dependency and bounded response variables, which occur in fact, are addressed jointly in this work. Relations of dependency which are generated because of the measure instrument, for instance the Reading comprehension test, are addresed including random effects. This effect models relations for specific structures of dependency caused as a result of applying the instrument. Relations because units of investigation are localized in the space can be modeled by a random effect, sui generis, in the spatial regression context. Bounded response variables are modeled using Beta, Simplex or Kumaraswamy distributions. This typical properties of the investigated datasets accomplish the purpose of suggesting a general model which cover simultaneously specific relations in the datasets and bounded responses, giving in this way enough models according to the realism obvious in the datasets studied in this work, as being responses for Reading comprehension, with the intention to have pragmatic inferences.

Keywords: Reading comprehension, bounded responses, testlet, spatial regression, Bayesian inference. 


\section{Sumário}

$\begin{array}{ll}\text { Lista de Figuras } & \text { ix }\end{array}$

Lista de Tabelas $\quad$ xi

1 Introdução $\quad 1$

2 Os modelos testlet para o estudo da compreensão leitora 5

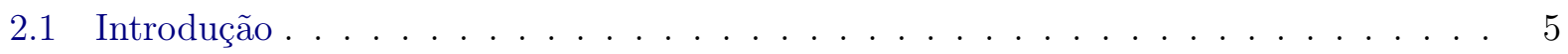

2.2 Os modelos da Teoria de Resposta ao Testlet (TRT) $\ldots \ldots \ldots$

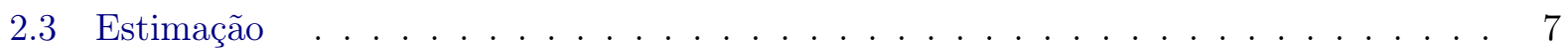

2.3.1 Função de Verossimilhança . . . . . . . . . . . . . . . . . . . . 8

2.3 .2 Distribuições a priori . . . . . . . . . . . . . . . . . . . . . . . . . 8

2.3.3 Distribuição a posteriori ． . . . . . . . . . . . . . . . . . . . . . 10

2.3 .4 Métodos MCMC . . . . . . . . . . . . . . . . . . . . . . . . 10

2.4 Estudo de simulação . . . . . . . . . . . . . . . . . . . . . . . . . 11

2.5 Aplicação . . . . . . . . . . . . . . . . . . . . . . . . . 13

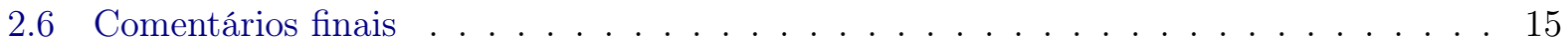

3 Modelos de regressão espacial com resposta limitada para o estudo da compreen$\begin{array}{ll}\text { são leitora } & 17\end{array}$

3.1 Introdução . . . . . . . . . . . . . . . . . . . . . . . . . . . . 17

3.2 O conjunto de dados e a análise preliminar . . . . . . . . . . . . . . . . . 18

3.3 Variáveis de resposta limitada . . . . . . . . . . . . . . . . . . . . . . . 21

3.4 O modelo de regressão espacial para respostas limitadas . . . . . . . . . . . . . . . 24

3.5 Inferência Bayesiana . . . . . . . . . . . . . . . . . . . 27

3.5.1 Critérios de comparação de modelos . . . . . . . . . . . . . . . . . 27

3.6 Simulações . . . . . . . . . . . . . . . . . . . . . . . . . . . . . . . . . 29

3.6.1 Estudo de recuperação . . . . . . . . . . . . . . . . . . . . . . . . . . . . 29

3.6.2 Estudo dos critérios de comparação dos modelos . . . . . . . . . . . . . . 30

3.7 Análise com dados reais . . . . . . . . . . . . . . . . . . . . . . . 30

3.8 Comentários finais . . . . . . . . . . . . . . . . . . . 36

4 Modelos do tempo de resposta limitado e a precisão da resposta para o estudo $\begin{array}{ll}\text { da compreensão leitora } & 37\end{array}$

4.1 Introdução . . . . . . . . . . . . . . . . . . . . . . . . 37

4.2 A distribuição limitada . . . . . . . . . . . . . . . . . . . . . . . 38 
4.3 Modelos para o tempo de resposta limitado (BRT) . . . . . . . . . . . . . . . 40

4.4 Modelo hierárquico conjunto para o tempo de resposta limitado e a precisão (HBRT) 42

4.5 Estimação Bayesiana . . . . . . . . . . . . . . . . . . . . . 43

4.6 Estudos de simulação . . . . . . . . . . . . . . . . . . . . . . . . . . . . 44

4.6.1 Recuperação dos parâmetros para o modelo do tempo de resposta limitado $(\mathrm{BRT}) \ldots \ldots \ldots \ldots \ldots \ldots \ldots \ldots 4 \ldots \ldots \ldots \ldots$

4.6.2 Recuperação dos parâmetros no modelo hierárquico conjunto para o tempo limitado e a precisão . . . . . . . . . . . . . . . . . . . . 45

4.7 Aplicação . . . . . . . . . . . . . . . . . . . . . . 47

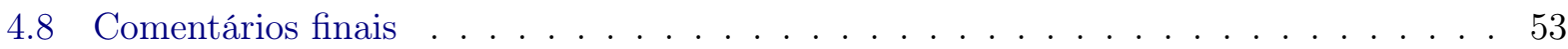

5 Conclusões $\quad 55$

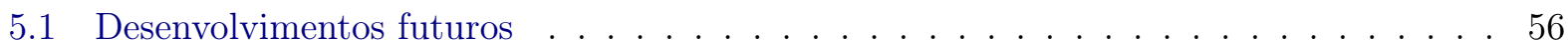

$\begin{array}{ll}\text { A O software STAN } & 57\end{array}$

A.1 Algoritmo Hamiltoniano Monte Carlo (HMC) . . . . . . . . . . . . . . . . . 57

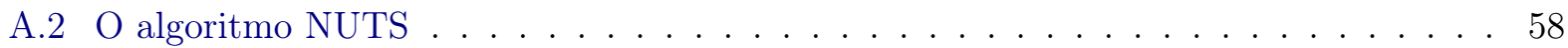

B Densidades da distribuição limitada $\quad 61$

$\begin{array}{ll}\text { C O método INLA } & 65\end{array}$

D Resultados adicionais no estudo de recuperação do capítulo $3 \quad 67$

E Resultados adicionais e o código usado na análise de dados reais do capítulo $3 \quad 71$

E.1 Resultados adicionais . . . . . . . . . . . . . . . . . . . . . . 71

E.2 código usado na análise de dados reais . . . . . . . . . . . . . . . . . . 71

F Códigos BUGS dos modelos HBRT-beta e HBRT-simplex $\quad 79$

F.1 Código BUGS do modelo HBR-beta . . . . . . . . . . . . . . . . . . . 79

F.2 Código BUGS do modelo HBRT-simplex . . . . . . . . . . . . . . . . . 80

Referências Bibliográficas $\quad 83$ 


\section{Lista de Figuras}

2.1 (a) histograma dos escores (pontuações totais) e (b) Proporção de respostas corretas 13

2.2 Estimativas dos parâmetros de discriminação e dificuldade para o modelo da TRT 2TP aplicado no conjunto de dados da CL. . . . . . . . . . . . . . . . . . . . . 14

3.1 Ajuste do modelo de regressão Beta: (a) resíduo padronizado $r_{i}$ versus a variável resposta estimada $\hat{y}_{i}$, (b) faixa de confiança da densidade preditiva a posteriori com o histograma do conjunto de dados CL . . . . . . . . . . . . . . . . . . . . 21

3.2 Mapa das 195 províncias peruanas junto com os resíduos, comportamento não explicado, do modelo de regressão Beta . . . . . . . . . . . . . . . . . . . . 22

3.3 Função de densidade de probabilidade da distribução limitada (beta, simplex e kumaraswamy) para distintos valores do parâmetro de posição $\theta$ e precisão $\phi . \quad$. . . . 25

3.4 Desvío padrão (sd) de uma variável com distribuição limitada como uma função do parâmetro de precisão $\phi$ e para uma quantidade fixa do parâmetro de locação $\theta$. . . 26

3.5 Ajuste do modelo SBDR-Ler Simplex para o conjunto de dados de CL. a) Resíduos padrão $\left(r_{i}\right)$ versus respostas ajustadas. b) Banda de confiança da distribuição preditiva a posteriori com o histograma da variável de resposta observada. . . . . . . 34

3.6 Mapa das 195 províncias peruanas para o conjunto de dados de CL com os resíduos padrão do modelo SBDR-Ler Simplex. . . . . . . . . . . . . . . . . . . . . . 34

3.7 Média a posteriori dos efeitos espaciais para o conjunto de dados de CL. . . . . . . . 35

4.1 Função de densidade de probabilidade das distribuições limitadas beta, simplex e kumaraswamy com distintas quantidades da posição $\theta$ e a precisão $\phi$. . . . . . . . . 41

4.2 Distribuição do tempo dos examinados, em segundos, para 28 itens selecionados de

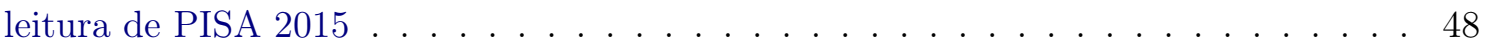

4.3 Distribuição do tempo limitado dos examinados em 28 itens selecionados de leitura de PISA $2015 \ldots \ldots \ldots \ldots$

4.4 Média e desvío padrão das respostas de acerto (exatidão) e tempo limitado para cada um dos 28 itens de leitura selecionados do PISA $2015 \ldots \ldots$. . . . . . . . . . 50

4.5 (a) Estimativa dos parâmetros dos items na TRI e (b) Estimativa dos parâmetros dos items no BRT para cada um dos 28 itens de leitura aplicados em PISA 2015, usando o modelo HBRT-beta. . . . . . . . . . . . . . . . . . . . . . . . . . 51

4.6 (a) Estimativa dos parâmetros dos items na TRI e (b) Estimativa dos parâmetros dos items no BRT para cada um dos 28 itens de leitura aplicados em PISA 2015, usando o modelo HBRT-simplex. . . . . . . . . . . . . . . . . . . . . . . 52 
4.7 Relação entre a rapidez limitada e a precisão na resposta para os avaliados em leitura em PISA 2015, usando o modelo HBRT-beta. . . . . . . . . . . . . . . . . . . 52

4.8 Relação entre a rapidez limitada e a precisão na resposta para os avaliados em leitura em PISA 2015, usando o modelo HBRT-beta. . . . . . . . . . . . . . . . . . . . 53

B.1 Função de densidade de probabilidade para a ditribuição limitada Beta. . . . . . . . . 62

B.2 Função de densidade de probabilidade para a distribuição limitada Simplex. . . . . . 63

B.3 Função de densidade de probabilidade para a distribuição limitada Kumaraswamy. . 64

E.1 Ajuste dos modelos Beta: Resíduos padrão $\left(r_{i}\right)$ vs respostas ajustads ( $\hat{y}$ ) (esquerda) e banda de confiança da PPD com o histograma observado (dereita). BR (parte superior), SBDR-Icar (no médio) and SBDR-Ler (parte inferior) . . . . . . . . . . . 73

E.2 Ajuste dos modelos Simplex: Resíduos padrão $\left(r_{i}\right)$ vs respostas ajustads $(\hat{y})$ (esquerda) e banda de confiança da PPD com o histograma observado (dereita). BR (parte superior), SBDR-Icar (no médio) and SBDR-Ler (parte inferior). . . . . . . . 74

E.3 Ajuste dos modelos Kumaraswamy: Resíduos padrão $\left(r_{i}\right)$ vs respostas ajustads $(\hat{y})$ (esquerda) e banda de confiança da PPD com o histograma observado (dereita). BR (parte superior), SBDR-Icar (no médio) and SBDR-Ler (parte inferior). . . . . . . . 75 


\section{Lista de Tabelas}

2.1 Estimativa do tamanho efetivo da amostra por segundo $(\hat{\delta})$ para o modelo da TRT $2 \mathrm{TP}$ usando os algoritmos HMC-NUTS e M-H. . . . . . . . . . . . . . . . 12

2.2 Estrutura dos Testlets para o conjunto de dados da Compreensão Leitora . . . . . . 13

2.3 WAIC para os modelos da TRT ajustados no conjunto de dados da CL . . . . . . . 14

2.4 Estimativas a posteriori do modelo testlet probito de dois parâmetros $(2 \mathrm{TP})$. . . . 15

3.1 Estadísticas Descriptivas para a variável resposta e as covariáveis do conjunto de dados CL. . . . . . . . . . . . . . . . . . . . . . . . . . 19

3.2 Estimativas dos parâmetros no modelo de regressão Beta para os dados de Compreensão Leitora . . . . . . . . . . . . . . . . . . . . . . 20

3.3 Estudo de recuperação dos parâmetros para o modelo simulado SBDR-Ler, considerando três distribuições limitadas e precisão espacial $\tau=1 \ldots$. . . . . . . . 31

3.4 Avaliação dos critérios de comparação de modelos DIC, WAIC e MAE para selecionar o verdadeiro modelo nos dados simulados do modelo SBDR-Ler considerando respostas das diferentes distribuições limitadas com precisão espacial $\tau=1$. As quantidades são o número de vezes em 100 conjuntos de dados. . . . . . . . . . . . . . . 31

3.5 Número de vezes selecionado como o melhor modelo sob os critérios DIC, WAIC e MAE, para modelo simulado SBDR-Ler com uma precisão espacial de $\tau=1$. . . . 32

3.6 Média à posteriori (média), desvio padrão (DP) e o $95 \%$ do intervalo de credibilidade (IC) para os parâmetros estimados do conjunto de dados RC sob os modelos SBDR-Ler 33

4.1 Resultados do estudo de simulação do modelo BRT para as distribuições limitadas beta e simplex. . . . . . . . . . . . . . . . . . . . . . . 45

4.2 Resultados da recuperação de parâmetros no modelo HBRT com diferentes distribuições limitadas . . . . . . . . . . . . . . . . . . . . . . . . . . . . . 46

D.1 Estudo de recuperação dos parâmetros para o modelo simulado SBDR-Ler, considerando três distribuições limitadas e precisão espacial $\tau=0.5 \ldots$. . . . . . 68

D.2 Estudo de recuperação dos parâmetros para o modelo simulado SBDR-Ler, considerando três distribuições limitadas e precisão espacial $\tau=2 \ldots \ldots$. . . . . . 69

E.1 Média a posteriori (Média), desvío padrao (DP) e o intervalo de credibilidade (IC) de 95\% para os parâmetros estimados dos dados de CL considerando diferentes modelos espaciasi para variáveis de respostas limitadas, sob os modelos SBDR-Icar, BR e SPOCK. 


\section{Capítulo 1}

\section{Introdução}

A compreensão leitora é uma habilidade fundamental, pois uma pessoa precisa em qualquer momento ler frases, questões, ou artigos escritos para a resolução de situações cotidianas. Nesse contexto, o processo da leitura foi estudado inicialmente analisando o tempo, a fisiologia e a memória como formas experimentais iniciais antes do estudo da psicologia da leitura, veja Huey (1898). Especificamente, estes estudos investigaram o tempo em que o examinado lê um texto considerando incluir varias colunas pequenas em comparação a amplidão habitual do texto, ademais se examina formas de movimento dos óculos e também o reconhecimento de palavras lidas previamente. Posteriormente, o autor Thorndike (1917) estudou o raciocínio da leitura, nesse estudo se sugere que a compreensão ao ler um parágrafo tem uma conexão com a resolução de um problema na matemática. Isto é, consiste em selecionar os elementos certos de uma situação e pôr juntos com relações adequadas, e com quantidades de peso ou influencia ou força adequadas. Um resumo desde os inicios da compreensão e competência leitora é estudado por Perez (2014).

Os modelos estatísticos apresentados na literatura, os quais consideram a medição da habilidade de compreensão leitora estão relacionados com os modelos da Teoria de Resposta ao Item (TRI) unidimensionais ou multidimensionais van der Linden (2016), os modelos Testlet Wang et al. (2002) e os modelos de diagnóstico cognitivo Jang (2009), von Davier (2008). Estudos recentes sugerem modelos conjuntos os quais utilizam, além da resposta ao item, o tempo de resposta, com a intenção de estimar a habilidade em compreensão leitora, veja Fox et al. (2007); Im (2015); Klein Entink (2009); van der Linden (2007); Zhan et al. (2018). Porém, o tempo de resposta utilizando tais modelos supõe que o tempo é infinito, mas na pratica o tempo é limitado. Nesse sentido, o presente trabalho abrange modelos estatísticos para os dados de compreensão leitora os quais contém relações de dependência e consideram o uso do tempo de resposta limitado.

No modelamento de dados geralmente supõe-se que um conjunto de respostas observadas são independentes. Isto é, assume-se a suposição de independência, a qual admite de forma simples o calculo da função de verosimilhança, a partir da qual são estimados os parâmetros de um modelo específico. Porém, na realidade, quando é analisado um determinado conjunto de dados, a suposição de independência resulta controversa. Por exemplo, na análise psicométrica, onde temos como propósito conhecer a habilidade ou traço latente de um individuo ou examinado e estimativas 
relacionadas com os itens ${ }^{1}$, assume-se independência entre as respostas de um conjunto de perguntas as quais têm um estímulo comum. Isto é, considera-se independência em respostas testlet, onde o testelt é desenhado de forma conjunta e as respostas estão relacionadas. Ademais, em outro contexto, quando é analisada informação de um conjunto de unidades, como as cidades ou regiões, as quais têm relações espaciais, supõe-se que as medições dos dados em diferentes cidades não estão relacionadas quando elas compartem idiossincrasias comuns com cidades vizinhas.

$\mathrm{Na}$ análise psicométrica, especificamente nos modelos de teoria de resposta ao item (TRI), geralmente são analisadas respostas de várias pessoas ou indivíduos ou examinados a um conjunto de itens de uma prova com a finalidade de medir um construto ou traço latente. Define-se o traço como a proficiência em leitura, a aptidão física de um esporte particular, a propensão à ansiedade, entre outros. Nesta análise é usual supor que as respostas dado o traço latente são independentes. Porém, a construção da prova nem sempre garante a independência das respostas. Pois, por exemplo, nos testes de compreensão leitora geralmente apresentam um texto e depois um conjunto de perguntas relacionadas com o texto, tal estrutura, denominada testlet, não preserva independência das respostas do individuo. Assim, em provas as quais contém uma estrutura testlet a suposição de independência é controversa. Respostas de itens agrupados com um estímulo comum motivaram o estudo dos modelos testlet, o qual considera um efeito aleatório no preditor linear latente no modelo TRI logístico de dois parâmetros, veja Bradlow et al. (1999). No modelo testlet, além de modelar a probabilidade da exatidão da resposta ao item, modela-se a interação da pessoa com os itens no interior do testlet e se fornece uma medida da relação entre as respostas, do examinado, aos itens no interior de um testlet. Uma comparação dos métodos de estimação nos modelos testlet é apresentada no capítulo 2 .

$\mathrm{Na}$ avaliação do progresso de políticas públicas em Educação, implementadas em municípios ou cidades, sugere fazer um seguimento de medidas como o nível da competência leitora de um grupo de estudantes. Geralmente, tais medidas são taxas ou proporções com quantidades que estão na faixa $(0,1)$, então ferramentas estatísticas são necessárias para modelar respostas limitadas no intervalo unitário $(0,1)$. Com tudo, as cidades têm uma localização espacial e elas compartilham características comuns com cidades próximas. Tal estrutura espacial não esta sendo considerada no modelamento dos dados limitados no intervalo unitário. Assim, considerando as peculiaridades compartilhadas entre cidades vizinhas, o capítulo 3 inclui um efeito aleatório sui generis, o qual encapsula a dependência espacial, nos modelos de regressão para respostas limitadas no intervalo unitário. Define-se o efeito aleatório sui generis, o qual introduz ate dois parâmetros no modelo de regressão limitado, utilizando as propriedades dos campos aleatórios de Markov gaussianos (GMRF) (Rue e Held, 2005), particularmente a definição Intrínseca condicional (ICAR) e a definição de Leroux Leroux et al. (2000). Os parâmetros adicionais no modelo de regressão fornecem estimativas da relação entre cidades as quais compartem idiossincrasias comuns com cidades em torno delas.

$\mathrm{Na}$ analise psicométrica, com o progresso dos sistemas computacionais e o uso da tecnologia, a obtenção de informação é ilimitada. Na atualidade, vários sistemas de avaliação utilizam computadores para investigar o desempenho em distintas áreas do conhecimento. Ademais, a recolecção de

\footnotetext{
${ }^{1}$ Item é definido como uma pergunta de uma prova
} 
informação através do uso de aplicações em aparelhos computacionais são mais simples. Nesse contexto, os tempos de resposta são naturalmente coletados e o desafio direciona apresentar modelos para analisar o tempo de resposta. Geralmente, o tempo é analisado como sendo uma variável ilimitada. No entanto, o tempo é limitado. Assim, formas alternativas para modelar o tempo limitado é sugerida no capítulo 4, considerando analisar o tempo como uma variável limitada no intervalo unitário. Logo, a proposta de modelar o tempo limitado pode ser adicionado ao modelo da exatidão na resposta ao item, de maneira conjunta, para estimar o traço latente da rapidez limitada e a precisão (exatidão na resposta). Esta forma de modelar duas variáveis respostas é denominada como modelo hierárquico conjunto. O qual, além de estimar os parâmetros do item, estima o traço latente da rapidez e a precisão, assumindo relações entre tais traços latentes.

Na definição de Inferência, a qual se define como a redução de incerteza, a abordagem Bayesiana fornece uma pratica perfeita de tal definição. Pois, esta abordagem sugere uma incerteza como uma definição a priori chamada a distribuição à priori e depois do experimento, quando são conhecidos os dados, define-se a verossimilhança e se alcança uma redução de tal incerteza com a definição da distribuição à posteriori. Assim, ao longo do presente trabalho a estimação dos parâmetros é aproximada através da inferência Bayesiana. Existem desafios nesse sentido, pois o uso dos métodos de estimação mediante métodos de cadeias de Markov Monte Carlo (MCMC) tornam-se demorado para modelos mistos onde são considerados efeitos aleatórios para modelar as relações presentes nos dados. Vários métodos de estimação são utilizados na abordagem Bayesiana, especificamente neste trabalho são considerados os algoritmos de Metrópolis-Hastings, NUTS e a aproximação INLA.

A verossimilhança é definida como uma função a qual relaciona um parâmetro a ser estimado com o conjunto de dados coletados como resultado de um experimento, específicamente com a estatística suficiente dos dados analisados. Assim, define-se a função de densidade de probabilidade (fdp) conjunta dos dados coletados e logo de conhecer os dados a fdp conjunta torna-se a função do parâmetro a ser estimado (a verossimilhança). Asume-se, ao longo do presente trabalho, a definição de permutabilidade, veja Schervish (1995), das respostas de tal forma que é possível obter a verossimilhança como o produto das fdp de cada uma das variáveis aleatórias as quais compõem os dados ou a amostra analisada.

O conteúdo do presente trabalho é desenvolvido da seguinte forma: No capítulo 2, o conceito de testlet é introduzido para modelar a habilidade da compreensão leitora (CL) e se estuda o modelo testlet para os dados de CL. No capítulo 3, apresenta-se novos modelos de regressão considerando distribuições de resposta limitada para dados de compreensão leitora no contexto espacial. No capítulo 4, usando algumas das distribuições de resposta limitada que foram apresentadas no capítulo 3 , se sugere a ideia de tempo de resposta limitada, assim um novo modelo de tempo de resposta limitada e precisão na resposta para dados de compreensão leitora é investigado. No capítulo 5 apresentamos conclusões dos estudos desta pesquisa e desenvolvimentos futuros são apresentados. Finalmente, nos apêndices A, B, C, D, E e F são apresentados materiais adicionais do desenvolvimento da presente tese.

Os capítulos da presente tese são manuscritos desenvolvidos desde o início do doutorado (2015). O 
manuscrito do capítulo 2 foi apresentado como pôster no V CONBRATRI (Congresso Brasileiro da Teoria de Resposta ao Item), realizado em Campinas-SP, em dezembro de 2016, na Universidade São Francisco (USF). A versão em inglês do capítulo 3 compõe o artigo Spatial regression models for bounded response variables with evaluation of the degree of dependence, elaborado conjuntamente com os professores Marcos Prates, Jorge Bazán e Heleno Bolfarine, o qual foi aceito na revista Statistics and its interface e esta na etapa de edição. Uma primeira versão das ideias iniciais do capítulo 4 é material do paper $A$ hierarchical joint model for bounded response time and response accuracy, produzido de maneira conjunta com os professores Jorge Bazán e Heleno Bolfarine, e foi aceito como uma contribuição no IMPS Procedings do Quantiative Psychology: 84th Annual Meeting of the Psychometric Society, Santiago, Chile, 2019.

A leitura da presente tese pode ser aleatória, ela foi escrita com o propósito de brindar ao leitor liberdade para conseguir informação específica ao longo dos capítulos que compõem a tese. 


\section{Capítulo 2}

\section{Os modelos testlet para o estudo da compreensão leitora}

\subsection{Introdução}

Teslet é uma definição sugerida por Wainer e Kiely (1987) a qual faz referencia a um conjunto de itens que são desenvolvidos como uma unidade e são administrados juntos (Wainer et al., 2007a). Por exemplo, em uma prova de compreensão leitora pequenos grupos de itens estão relacionados com um texto, em uma prova de matemática conjuntos de itens estão ligados com um gráfico ou tabela, ou em estudos sociais, grupos de itens os quais estão relacionados com um mapa. Conhecese da literatura que a presença de testlets pode infringir a suposição de independência condicional dos itens, os quais compartem material de forma explícita, veja Rosenbaum (1988). A suposição de Independência Condicional (IC) dos itens significa que as respostas dos itens estão relacionadas uns com outras só a través da variável latente (Lord e Novick, 1968), assim itens associados em um testlet não satisfazem o suposto de IC. Quando estruturas de testlet são observadas e ignoradas, usando os modelos de Teoría de Resposta ao Item (TRI) padrão, com a suposição de independência condicional, o resultado será sobre-estimação da precisão do traço latente e ademais presença de viés nas estimativas dos parâmetros de dificuldade e discriminação, veja (Bradlow et al., 1999).

Nesse contexto, Bradlow et al. (1999) desenvolveram uma aproximação paramétrica para modelar e avaliar o grau de dependência condicional dos itens em um testlet sob uma perspectiva de modelamento Bayesiano, especificamente, os autores consideraram o modelo TRI com dois parâmetros (relacionados com a dificuldade e a discriminação) e acrescentaram um efeito aleatório que da conta da dependência dos itens no interior de um testlet. Apos o trabalho de Bradlow vários outros modelos testlets foram formulados, por exemplo: Um modelo geral bayesiano para testlets, veja (Wang et al., 2002), neste modelo se apresenta uma extensão do modelo de Bradlow incluindo o parâmetro de acerto casual, outro modelo desenvolvido foi o modelo Rash testlet (Wang e Wilson, 2005) o qual analisa o modelo Rash considerando o efeito testlet, temos também a extensão do modelo Rash testlet (Paek et al., 2009) onde os autores sugerem uma relação do traço latente com os efeitos testlet, outro modelo apresentado é o modelo logístico de exponente positivo (Flores Ari, 2012) onde a probabilidade de acerto ao item condicionado aos parâmetros do modelo tem uma distribuição assimétrica, e ademais entre outros modelos temos o aprimoramento do modelo de 
resposta ao testlet dos parâmetros com a adição do parâmetro de discriminação testlet (Tao et al., 2013).

Desde uma perspectiva de modelamento bayesiano, os autores Wainer et al. (2007a) definiram os modelos da Teoria de Resposta ao Testlet (TRT) os quais estão relacionados com os modelos da TRI e incluem o efeito aleatório testlet, tais modelos podem naturalmente ser ajustados utilizando o programa WinBUGS, veja (Spiegelhalter et al., 2003a). Por exemplo, é possível seguir o código BUGS desenvolvido e disponível no trabalho de McKay (2010). Encontra-se também o software SCORIGHT elaborado pelo autor Wang et al. (2005), o qual ajusta alguns modelos TRT no âmbito bayesiano usando simulação de Monte Carlo via cadeias de Markov (MCMC) e ao nível do usuário o software é manipulado no entorno DOS.

O método BUGS (do acrônimo Bayesian inference Using Gibbs Sampling) utiliza simulação de amostras MCMC, mas na procura de convergência o proceso de obtenção de amostras maiores podese tornar demorado, ademais tais amostras estão correlacionadas e em consequência a escolha de uma amostra sistemática não correlacionada é necessária (Levy, 2009). Um método alternativo, o qual foi proposto por Duane et al. (1987), é o mecanismo de simulação Hamiltoniano Monte Carlo (HMC). Esta simulação fornece uma exploração eficiente e necessária em modelos hierárquicos complexos, veja (Betancourt e Girolami, 2013). Atualmente existe um recente desenvolvimento computacional denominado Stan (Stan-Development-Team, 2016) o qual utiliza amostras HMC considerando o amostrador No-U-Turn (NUTS). Os usuários do Stan podem elaborar, testar e rodar modelos complexos sem o compromisso das limitações computacionais. Nesse sentido, apresenta-se neste capítulo o ajuste dos modelos TRT utilizando o algoritmo NUTS e avalia-se o desempenho em comparação ao conhecido algoritmo de Metropolis-Hastings (M-H) do WinBUGS.

O esquema do presente capítulo é organizado da seguinte forma: Na seção 2, apresentam-se os modelos TRT o qual abrange os modelos que consideram o efeito testlet. Os detalhes da estimação dos parâmetros dos modelos são mostrados na seção 3. Um estudo de simulação com a intenção de investigar o desempenho do algoritmo NUTS em relação ao algoritmo de M-H apresenta-se na seção 4. Na seção 5, aplica-se o algoritmo NUTS em um conjunto de dados de uma prova de compreensão leitora e finalmente as conclusões e desenvolvimentos futuros são sugeridos na seção 6 .

\subsection{Os modelos da Teoria de Resposta ao Testlet (TRT)}

Os modelos da Teoria de Resposta ao Testlet (TRT) consideram os modelos da Teoria de Resposta ao Item (TRI) e incluem um efeito aleatório denominado como efeito testlet, o qual modela a relação das respostas aos items no interior de um testlet. Isto é, o efeito testlet retém o excesso de covariância no interior de um testlet, veja (Wainer et al., 2007b). A seguir apresentamos a definição do modelo testlet de dos parâmetros.

Assume-se um conjunto de respostas observadas de uma prova, onde $Y_{i j}$ define a resposta de um individuo em um item, em que $i$ denota o individuo avaliado $(i=1, \ldots, n)$ e $j$ simboliza o item da prova $(j=1, \ldots, J)$. Tais respostas têm um escore de 0 se o individuo não acertou o item ou 1 se o individuo acertou o item. Define-se que cada uma das respostas $Y_{i j}$ segue uma distribuição 
condicional da seguinte forma:

$$
Y_{i j} \sim f\left(y_{i j} \mid \theta_{i}, a_{j}, b_{j}, \gamma_{i l}\right)
$$

onde $f\left(y_{i j} \mid \theta_{i}, a_{j}, b_{j}, \gamma_{i l}\right)=\left[\Phi\left(a_{j}\left(\theta_{i}-b_{j}-\gamma_{i l}\right)\right)\right]^{y_{i j}}\left[1-\Phi\left(a_{j}\left(\theta_{i}-b_{j}-\gamma_{i l}\right)\right)\right]^{1-y_{i j}}$ simboliza a distribuição Bernoulli com a probabilidade da resposta correta $P\left(Y_{i j}=1 \mid \theta_{i}, a_{j}, b_{j}, \gamma_{i l}\right)=p_{i j}=\Phi\left(a_{j}\left(\theta_{i}-b_{j}-\gamma_{i l}\right)\right)$.

A função $\Phi($.$) é definida como uma função de distribuição acumulada (fda) e denominada como$ função de ligação. Podemos considerar a fda da normal padrão e em consequência o modelo é denominado como modelo testlet probito ou também podemos considerar a fda da distribuição logística e o modelo é denominado como modelo testlet logito. As variáveis $a_{j}>0$ e $b_{j} \in \mathbb{R}$ simbolizam os parâmetros do item, denominados como de discriminação e dificuldade, respectivamente; $\theta_{i} \in \mathbb{R}$ é nomeado como traço latente e assume-se que ele é aleatório com distribuição normal padrão, isto é, $\theta_{i} \sim \mathcal{N}(0,1)$, veja van der Linden (2016) vol 1 cap. 2 ; ademais, o $\gamma_{i l} \in \mathbb{R}$ é o efeito aleatório testlet e assume-se que tem distribuição normal com média zero y variância $\sigma_{l}^{2}$, isto é, $\gamma_{i l} \sim \mathcal{N}\left(0, \sigma_{l}^{2}\right)$, veja (Bradlow et al., 1999), em que o $l$ é o indicador do testlet $(l=1, \ldots, L)$ e $\sigma_{l}^{2}$ é o parâmetro que da conta do efeito testlet e quando ele atinge um maior valor significa que existe um maior efeito testlet.

Os modelos testlet definidos mediante a equação 2.1 consideram dois parâmetros do item e serão denotados como 2TL para o modelo testlet logito e $2 \mathrm{TP}$ para o modelo testlet probito de dois parâmetros. Quando não é considerado no modelo o parâmetro $a_{j}$, o modelo é nomeado como modelo testlet logito de um parâmetro 1TL ou modelo testlet probito de um parâmetro 1TP.

\subsection{Estimação}

Na presente seção desenvolve-se a estimação dos parâmetros para o modelo testlet de dois parâmetros, definido na seção 2.2. Em relação aos métodos de estimação para modelos testlet temos que eles estão relacionados às estimações dos métodos da Teoría de resposta ao Item (TRI), pois se note que o modelo testlet é um modelo TRI com a adição do efeito testlet. Na revisão geral dos métodos de estimação na TRI podemos citar o handbook da TRI (Volume 2) van der Linden (2016) ou a dissertação de Azevedo (2003).

Nesse sentido, em relação aos modelos TRT temos que a estimação através do método de Maximização Marginal Conjunta (MMC) não é aplicada na prática, o método via Máxima Verossimilhança Marginal (MVM) exige confiança assintótica da distribuição Normal. No método MVM maximiza-se a seguinte verossimilhança marginal (VM) Wainer et al. (2007a)

$$
L\left(\boldsymbol{a}, \boldsymbol{b}, \boldsymbol{\sigma}_{\gamma}^{2} \mid \boldsymbol{y}\right)=\iint \prod_{i=1}^{I} \prod_{j=1}^{J} p_{i j}^{y_{i j}}\left(1-p_{i j}\right)^{\left(1-y_{i j}\right)} d \boldsymbol{F}(\theta) d \boldsymbol{F}(\gamma),
$$

onde integra-se em relação ao traço latente $(\boldsymbol{\theta})$ e o efeito testlet $(\boldsymbol{\gamma})$. Neste procedimento, temos que a integral não pode ser obtida de forma fechada, inclusive quando é possível obter a VM o processo de obtenção das estimativas pontuais precisa de duas etapas: a primeira etapa esta em relação à estimação dos parâmetros do item $(\boldsymbol{a}, \boldsymbol{b})$ e a variância do efeito testlet $\left(\boldsymbol{\sigma}_{\gamma}^{2}\right)$ e a segunda etapa fixa os valores estimados obtidos na primeira etapa e estima $\boldsymbol{\theta}$ e o efeito testlet $(\boldsymbol{\gamma})$. Com tudo, as estimativas pontuais obtidas têm erros padrão os quais são calculados usando aproximações 
assintóticas da distribuição Normal.

No presente trabalho adota-se a estimação da Abordagem Bayesiana Plena (ABP), neste processo de estimação as distribuições aposteriori, conjuntas e marginais, dos parâmetros de interesse são obtidas de modo empírico, veja Azevedo (2008), e utiliza a verossimilhança aumentada, veja Albert (1992). Assim, considerando o desenvolvimento de importantes ferramentas computacionais, nas próximas seções utiliza-se a ABP usando a verossimilhança condicional para a estimação dos parâmetros do modelo testlet definido na seção 2.2 o qual considera a estimação de dois parâmetros associados ao item.

\subsubsection{Função de Verossimilhança}

Definimos a seguir a Função de Verossimilhança. Considerando a distribuição conjunta condicional do conjunto de dados observados, $f(\boldsymbol{y} \mid \boldsymbol{\beta}, \boldsymbol{\theta}, \boldsymbol{\gamma})$, a qual é uma função que relaciona o vector aleatório $\boldsymbol{Y}$ com os parâmetros do modelo, logo dos dados serem observados a funcão $f$ é denominada como a Função de Verossimilhança (FV), veja Schervish (1995) Pág. 13 ou Gelman et al. (1995) Pág. 9. Para o modelo previamente apresentado na equação (2.1), define-se a FV como a seguir:

$$
L(\boldsymbol{\beta}, \boldsymbol{\theta}, \boldsymbol{\gamma} \mid \boldsymbol{y})=\prod_{i=1}^{I} \prod_{j=1}^{J} p_{i j}^{y_{i j}}\left(1-p_{i j}\right)^{\left(1-y_{i j}\right)}
$$

onde $\boldsymbol{\beta}=\left(\boldsymbol{a}^{\prime}, \boldsymbol{b}^{\prime}\right)^{\prime}$ denota os parâmetros dos itens em que $\boldsymbol{a}^{\prime}=\left(a_{1}, \ldots, a_{J}\right)$ e $\boldsymbol{b}^{\prime}=\left(b_{1}, \ldots, b_{J}\right)$; $\boldsymbol{\theta}^{\prime}=\left(\theta_{1}, \ldots, \theta_{I}\right)$ denota o traço latente dos indivíduos e $\boldsymbol{\gamma}^{\prime}=\left(\gamma_{11}, \ldots, \gamma_{I L}\right)$ denota o efeito aleatório testlet de cada indivíduo para cada um dos $L$ testlets. É importante salientar que as respostas dos indivíduos para o modelo testlet têm independência condicionada ao conhecimento do traço latente, $\boldsymbol{\theta}$ (habilidade do individuo), e ao efeito testlet $\boldsymbol{\gamma}$, os quais são considerados como informação a priori no modelo.

\subsubsection{Distribuições a priori}

A eleição da distribuição a priori na analise Bayesiana é uma etapa controversa, mas a escolha apropriada da distribuição a priori pode resultar em estimações mais precisas, veja Jhonson e Sinharay no van der Linden (2016) vol. 2 capitulo 13. A escolha das prioris para a análise do presente capítulo esta baseada na revisão da literatura em relação aos modelos testlet e modelos da TRI, também com a intenção de obter identificabilidade no modelo e ademais considerações do espaço paramétrico. Nesse sentido, definem-se as distribuições a priori independentes para os parâmetros a serem estimados no modelo, da seguinte forma:

$$
p\left(\boldsymbol{\beta}, \boldsymbol{\theta}, \boldsymbol{\gamma} \mid \boldsymbol{\zeta}_{\boldsymbol{\beta}}, \boldsymbol{\zeta}_{\boldsymbol{\theta}}, \boldsymbol{\zeta}_{\boldsymbol{\gamma}}\right)=\prod_{j}^{J} p\left(\beta_{j} \mid \zeta_{\beta}\right) \times \prod_{i}^{I} p\left(\theta_{i} \mid \zeta_{\theta}\right) \times \prod_{i}^{I} \prod_{l}^{L} p\left(\gamma_{i l} \mid \zeta_{\gamma}\right),
$$

onde, $p\left(\beta_{j} \mid \zeta_{\beta}\right)=p\left(a_{j} \mid \zeta_{a}\right) p\left(b_{j} \mid \zeta_{b}\right)$ simboliza a distribuição a priori para os parâmetros de discriminação e dificuldade do item $j, \zeta_{a}$ e $\zeta_{b}$ são nominados como hiperprioris e elas podem ser fixas ou aleatórias, considera-se neste estudo hiperprioris fixas para os parâmetros dos items. Nosso caso, em que usamos a $\mathrm{ABP}, p\left(\theta_{i} \mid \zeta_{\theta}\right)$ é a distribuição a priori do traço latente para o i-ésimo individuo e $p\left(\gamma_{i l} \mid \zeta_{\gamma}\right)$ é a distribuição a priori do efeito testlet para o individuo $i$ no testlet $l$. De forma semel- 
hante aos parâmetros do item, $\zeta_{\theta}$ e $\zeta_{\gamma}$ são as hiperprioris de $\theta$ e $\gamma$ respetivamente e consideramos hiperprioris fixas para o traço latente $\theta$ e uma hiperpriori aleatória é definida para $\gamma$. Assim, a equação 2.4 é reduzida da seguinte forma:

$$
p\left(\boldsymbol{\beta}, \boldsymbol{\theta}, \boldsymbol{\gamma} \mid \boldsymbol{\zeta}_{\boldsymbol{\gamma}}\right)=\prod_{j}^{J} p\left(\beta_{j}\right) \times \prod_{i}^{I} p\left(\theta_{i}\right) \times \prod_{i}^{I} \prod_{l}^{L} p\left(\gamma_{i l} \mid \zeta_{\gamma}\right),
$$

Na TRI, algumas distribuições a priori tradicionais foram comparadas com prioris não informativas e prioris hierárquicas, veja Natesan et al. (2016), o estudo sugere boas estimativas relacionadas ao uso de prioris tradicionais e hierárquicas em relação ao uso de prioris não informativas. Assim, no presente capítulo utiliza-se prioris tradicionais para os parâmetros do item e o traço latente, tais distribuições a priori são definidas como a seguir:

$$
\begin{aligned}
a_{j} & \sim \log \operatorname{Normal}(0, .25) \\
b_{j} & \sim \mathcal{N}(0,1) \\
\theta_{i} & \sim \mathcal{N}(0,1)
\end{aligned}
$$

A eleição das distribuições a priori está também em relação ao espaço paramétrico dos parâmetros. Temos no caso do parâmetro de discriminação o espaço paramétrico é $a>0$ e as estimativas geralmente estão ao redor de 1, então assumimos uma distribuição logNormal com uma média, em escala logarítmica, de zero; e um desvio padrão, também em escala logarítmica, de 0.25 com a intenção de brindar certa informação na estimação do modelo. Podemos também usar uma priori hierárquica como é sugerida no trabalho de Natesan et al. (2016), mas no presente trabalho usamos uma priori informativa pois podemos enxergar o rango de valores onde estão as estimativas do parâmetro de discriminação. Enquanto ao parâmetro de dificuldade $b$, existe um consenso no uso da distribuição normal como distribuição a priori, usamos assim uma distribuição a priori normal padrão com a intenção de permitir que o parâmetro da dificultade varie na faixa dos reais no intervalo $(-4,4)$.

O traço latente $\theta$ é considerado como um efeito aleatório, em consequência o traço latente segue uma distribuição normal padrão, com média zero e variância um. Além disso, esta eleição permite a identificabilidade do modelo.

Em relação ao efeito testlet, o qual também é um efeito aleatório, assume-se que ele segue uma distribuição a priori normal com média zero, com a intenção de estabelecer o efeito como um desvio do zero, e variância $\sigma_{\gamma_{l}}^{2}$. Isto é,

$$
\gamma_{i l} \sim \mathcal{N}\left(0, \sigma_{\gamma_{l}}^{2}\right)
$$

onde, $\sigma_{\gamma_{l}}^{2}$ é o indicador da quantidade do efeito testlet atribuída ao $l$-ésimo testlet, veja Wang et al. (2002) e Bradlow et al. (1999). Define-se ademais uma hiperpriori para $\sigma_{\gamma_{l}}^{2}$, a qual é definida como $\sigma_{\gamma_{l}}^{2} \sim \operatorname{IG}\left(s^{\prime}, r^{\prime}\right)$, onde IG simboliza a distribuição Gamma inversa, esta distribuição a priori é equivalente se consideramos a precisão $v_{\gamma_{l}}=1 / \sigma_{\gamma_{l}}^{2} \sim \operatorname{Gamma}(s, r)$. Consideramos $s=0.25$ e $r=0.5$, onde $s$ e $r$ denotam os parâmetros "shape" e "rate" da distribuição Gamma. Análogamente, $v_{\gamma_{l}}=1 / \sigma_{\gamma_{l}}^{2} \sim \chi^{2}(0.5)$. A eleição dos valores $s$ e $r$ consideram uma faixa de valores onde a precisão 
$v_{\gamma_{l}}$ consegue uma distribuição a posteriori própria.

\subsubsection{Distribuição a posteriori}

Na presente seção, considera-se a função de verossimilhança e as distribuições à priori apresentadas previamente e calcula-se a distribuição a posteriori, a qual pode ser definida como a seguir:

$$
\begin{aligned}
p(\boldsymbol{\beta}, \boldsymbol{\theta}, \boldsymbol{\gamma} \mid \boldsymbol{y}) & =\frac{p(\boldsymbol{\beta}, \boldsymbol{\theta}, \boldsymbol{\gamma}, \boldsymbol{y})}{p(\boldsymbol{y})} \\
& \propto p(\boldsymbol{y} \mid \boldsymbol{\beta}, \boldsymbol{\theta}, \boldsymbol{\gamma}) p\left(\boldsymbol{\beta}, \boldsymbol{\theta}, \boldsymbol{\gamma} \mid \sigma_{\gamma_{l}}^{2}\right) p\left(\sigma_{\gamma_{l}}^{2}\right) \\
& =p(\boldsymbol{y} \mid \boldsymbol{\beta}, \boldsymbol{\theta}, \boldsymbol{\gamma}) p(\boldsymbol{\beta}) p(\boldsymbol{\theta}) p\left(\boldsymbol{\gamma} \mid \sigma_{\gamma_{l}}^{2}\right) p\left(\sigma_{\gamma_{l}}^{2}\right) \\
& =L(\boldsymbol{\beta}, \boldsymbol{\theta}, \boldsymbol{\gamma} \mid \boldsymbol{y}) p(\boldsymbol{a}) p(\boldsymbol{b}) p(\boldsymbol{\theta}) p\left(\boldsymbol{\gamma} \mid \sigma_{\gamma_{l}}^{2}\right) p\left(\sigma_{\gamma_{l}}^{2}\right),
\end{aligned}
$$

onde $L(. \mid$.) simboliza a função de verossimilhança definida na equação (2.3) e os demais termos na direita são as distribuições à priori definidas nas equações (2.5), (2.6) e (2.7). Deste modo, a distribuição a posteriori tem a seguinte expressão:

$$
\begin{aligned}
p(\boldsymbol{\beta}, \boldsymbol{\theta}, \boldsymbol{\gamma} \mid \boldsymbol{y}) & \propto \prod_{i=1}^{I} \prod_{j=1}^{J}\left[\frac{1}{\sqrt{2 \pi}} \int_{-\infty}^{a_{j}\left(\theta_{i}-b_{j}-\gamma_{i l}\right)} e^{-t^{2} / 2} d t\right]^{y_{i j}}\left[\frac{1}{\sqrt{2 \pi}} \int_{a_{j}\left(\theta_{i}-b_{j}-\gamma_{i l}\right)}^{\infty} e^{-t^{2} / 2} d t\right]^{1-y_{i j}} \\
& \times \prod_{j=1}^{J} \frac{2}{a_{j} \sqrt{2 \pi}} \exp \left\{-2\left(\log \left(a_{j}\right)\right)^{2}\right\} I_{(0, \infty)}\left(a_{j}\right) \frac{1}{\sqrt{2 \pi}} \exp \left\{-\frac{b_{j}^{2}}{2}\right\} \\
& \times \prod_{i=1}^{I} \frac{1}{\sqrt{2 \pi}} \exp \left\{-\frac{\theta_{i}^{2}}{2}\right\} \\
& \times \prod_{i=1}^{I} \prod_{l=1}^{L} \frac{1}{\sqrt{2 \pi \sigma_{\gamma_{l}}^{2}}} \exp \left\{-\frac{\gamma_{i l}^{2}}{2 \sigma_{\gamma_{l}}^{2}}\right\} \frac{0.5^{0.25}}{\Gamma(0.25)}\left[\frac{1}{\sigma_{\gamma_{l}}^{2}}\right]^{0.25-1} \exp \left\{-\frac{1}{2 \sigma_{\gamma_{l}}^{2}}\right\} .
\end{aligned}
$$

Esta distribuição a posteriori (2.9) não tem uma forma analítica tratável, em consequência consideramos para a estimação dos parâmetros os métodos MCMC.

\subsubsection{Métodos MCMC}

Define-se o método de Cadeias de Markov Monte carlo (MCMC) como a integral de Monte Carlo a qual utiliza cadeias de Markov. Neste método, a integral de Monte Carlo colhe amostras de uma distribuição requerida e calcula a média amostral com a intenção de estimar a esperança matemática. As cadeias de Markov Monte Carlo extraem as amostras de uma cadeia de Markov extensa e habilmente elaborada, veja Gilks et al. (1995). Existem várias formas de construir tais cadeias, mas todas são casos especiais da abordagem de Metropolis-Hastings (M-H). Assim, a seguir apresentamos o algoritmo de M-H.

Assume-se uma distribuição a posteriori objetivo $f(\boldsymbol{\theta} \mid \boldsymbol{y})$ da qual precisamos gerar amostras de tamanho $T$, onde $\boldsymbol{\theta}^{(t)}$ é o vetor das quantidades geradas na iteração $t$. O algoritmo M-H define as seguintes etapas:

1. Iniciar quantidades $\boldsymbol{\theta}^{0}$. 
2. Repetir para $t=1, \ldots, T$ os seguintes procedimentos:

- $\boldsymbol{\theta}=\boldsymbol{\theta}^{(t-1)}$

- Extrair quantidades candidatas para $\boldsymbol{\theta}^{\prime}$ da distribuição proposta $q\left(\boldsymbol{\theta}^{\prime} \mid \boldsymbol{\theta}\right)$.

- Calcular $\alpha=\min \left(1, \frac{f\left(\boldsymbol{\theta}^{\prime} \mid \boldsymbol{y}\right) q\left(\boldsymbol{\theta} \mid \boldsymbol{\theta}^{\prime}\right)}{f(\boldsymbol{\theta} \mid \boldsymbol{y}) q\left(\boldsymbol{\theta}^{\prime} \mid \boldsymbol{\theta}\right)}\right)$

- Extrair $u$ da distribuição Uniforme $(0,1)$

- Se $u<=\alpha$ então $\boldsymbol{\theta}^{(t)}=\boldsymbol{\theta}^{\prime}$ caso contrario $\boldsymbol{\theta}^{(t)}=\boldsymbol{\theta}$.

Observe que $f\left(\boldsymbol{\theta}^{\prime} \mid \boldsymbol{y}\right)=\frac{f\left(\boldsymbol{\theta}^{\prime}, \boldsymbol{y}\right)}{f(\boldsymbol{y})}$ e $f(\boldsymbol{\theta} \mid \boldsymbol{y})=\frac{f(\boldsymbol{\theta}, \boldsymbol{y})}{f(\boldsymbol{y})}$, então o termo $f(\boldsymbol{y})$ é anulado. Assim, temos que o $\alpha$ é reduzido como a seguir:

$$
\alpha=\min \left(1, \frac{f\left(\boldsymbol{y} \| \boldsymbol{\theta}^{\prime}\right) f\left(\boldsymbol{\theta}^{\prime}\right) q\left(\boldsymbol{\theta} \mid \boldsymbol{\theta}^{\prime}\right)}{f(\boldsymbol{y} \mid \boldsymbol{\theta}) f(\boldsymbol{\theta}) q\left(\boldsymbol{\theta}^{\prime} \mid \boldsymbol{\theta}\right)}\right)
$$

O algoritmo amostrador do Gibbs é um caso particular do $\mathrm{M}-\mathrm{H}$, o qual utiliza como densidade proposta $q\left(\boldsymbol{\theta}^{\prime} \mid \boldsymbol{\theta}\right)$, isto é, as distribuições a posteriori condicionais completas para cada $\theta_{i}$. Este algoritmo é implementado de forma predefinida no WinBUGS, porém quando não é possível identificar as condicionais completas o WinBUGS usa o algoritmo M-H.

Uma forma distinta de elaborar uma cadeia de Markov Monte Carlo é a través do algoritmo Hamiltoniano Monte Carlo (HMC) o qual desvia-se do comportamento de caminho aleatório e da sensibilidade em obter cadeias correlacionadas, em consequência este algoritmo apresentaria uma convergência mais rápida em relação ao tradicional algoritmo de M-H. Uma configuração do HMC é o algoritmo NUTS, do acrônimo em inglês No-U-Turn Sampler, este algoritmo evade o uso de parâmetros específicos no HMC, como são a quantidade de passos e o tamanho deles, veja (Hoffman e Gelman, 2014). No presente trabalho analisaremos a convergência dos parâmetros nos modelos testlet usando os algoritmos NUTS e M-H. O apêndice A apresenta detalhes dos algoritmo NUTS o qual esta implementeado no software STAN.

\subsection{Estudo de simulação}

Apresenta-se nesta seção um estudo de simulação o qual tem o intuito de comparar o desempenho do algoritmo HMC-NUTS implementado no software STAN, veja Carpenter et al. (2017); Stan-Development-Team (2016) em relação ao reconhecido método do Metropolis Hasting (M-H) implementado no WinBugs, veja Lunn et al. (2012), para o modelo testlet probito de dois parâmetros (2TP) definido na seção 2.2 .

Nesse propósito, simulamos três conjuntos de dados com diferentes tamanhos de amostra $(n=$ $100,500,1000)$ onde os $n$ indivíduos respondem 20 perguntas ou itens de uma prova, tais perguntas estão associados em 4 testlets. O teslet 1 contém os itens 2,3 e 4; o testlet 2 inclui os itens 5, 6 e 7; o testlet 3 compreende os itens 8, 9, 10 e 11; o testlet 4 compõe-se dos itens 12, 13, 14 e 15. Os itens 1 , 16,17,18 e 19 são itens independentes. Esta estrutura dos itens em testlets é adicionado no modelo mediante um vetor definido da seguinte forma: $T=(5,1,1,1,2,2,2,3,3,3,3,4,4,4,4,5,5,5,5,5)$ onde as quantidades no vetor $T$ simbolizam o número do testlet ao qual pertencem os itens, e os 
itens independentes assumem o valor $L+1=5$, sendo $L$ a quantidade de testlets presentes na prova.

Os valores dos parâmetros foram simulados das seguintes distribuições: $\theta \sim \mathcal{N}(0,1), a \sim$ $\mathcal{N}\left(0.8, \sigma_{a}^{2}=0.2^{2}\right), b \sim \mathcal{N}(0,1), \gamma_{i l} \sim \mathcal{N}\left(0, \sigma_{\gamma_{l}}^{2}\right)$ e $\sigma_{\gamma_{l}}^{2}=0.5$ para cada $l$. Ambos algoritmos têm definido quantidades inicias idênticas para os parâmetros $a, b, \theta$ e $\sigma_{\gamma_{l}}^{2}$. Consideram-se duas cadeias simuladas com 5000 iterações em cada algoritmo, onde 2500 iterações constituem a fase de inicialização e 2500 iterações compõem as amostras da distribuição a posteriori a serem utilizadas na estimação dos parâmetros.

A convergência foi avaliada com a medida estatística $\hat{R}$, a qual fornece uma taxa estimada da variância entre cadeias em relação à variância no interior das cadeias para um parâmetro específico. Atingir convergência perfeita significa que o $\hat{R}$ é igual a um, mas valores inferiores de 1.1 são considerados como aceitáveis, veja (Gelman et al., 1995) Pág. 332. Para avaliar a eficiência das estimativas utiliza-se o tamanho efetivo da amostra (TEA) (ESS, do acrônimo em inglês effective sample size) por segundo, isto é $\hat{\delta}=\hat{n}_{\text {eff }} / s$, veja (Grant et al., 2016). O tempo para ambos os algoritmos considera a fase de inicialização.

Tabela 2.1: Estimativa do tamanho efetivo da amostra por segundo $(\hat{\delta})$ para o modelo da TRT 2TP usando os algoritmos $H M C$-NUTS e $M-H$.

\begin{tabular}{|l|l|c|r|c|r|}
\hline \multirow{3}{*}{$n$} & \multirow{2}{*}{ Parâmetro } & \multicolumn{2}{|c|}{ Algoritmo HMC-NUTS } & \multicolumn{2}{c|}{ Algoritmo M-H } \\
\cline { 3 - 6 } & $a$ & $\hat{\delta}$ & segundos & $\hat{\delta}$ & segundos \\
\hline \multirow{5}{*}{500} & $b$ & 5.498 & 458 & 5.603 & 244 \\
& $\sigma_{\gamma_{d(j)}}^{2}$ & 8.127 & 458 & 10.398 & 244 \\
& $b$ & 0.667 & 458 & 0.737 & 244 \\
& $b$ & 0.860 & 2475 & 0.336 & 3188 \\
& $\sigma_{\gamma_{d(j)}}^{2}$ & 0.067 & 2475 & 0.543 & 3188 \\
\hline 1000 & $a$ & 0.282 & 2475 & 0.037 & 3188 \\
& $b$ & 0.409 & 5690 & 0.082 & 12241 \\
& $\sigma_{\gamma_{d(j)}}^{2}$ & 0.022 & 5690 & 0.132 & 12241 \\
& & & 5690 & 0.008 & 12241 \\
\hline
\end{tabular}

Os resultados do estudo de simulação são apresentados na tabela 2.1. A quantidade $\hat{n}_{\text {eff }}$ foi calculada usando a função multiESS() do pacote mcmcse no R, veja Flegal et al. (2017). A estimativa

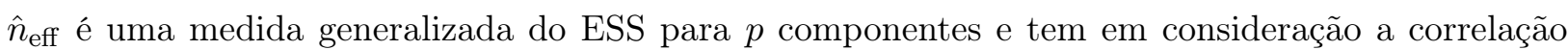
dos $p$ componentes, tal medida foi sugerida no trabalho da análise das saídas multivariadas para as cadeias de markov Monte Carlo, veja Vats et al. (2015). Podemos observar na Tabela 2.1, a estimativa do tamanho efetivo de amostras por segundo, $\hat{\delta}$, a qual mostra que quando $n$ cresce o tamanho efetivo de amostras simuladas é maior no algoritmo HMC-NUTS e ademais o tempo em obter tais amostras é menor, este resultado sugere um melhor desempenho para o algoritmo HMC-NUTS quando o tamanho da amostra $n$ é maior. Confirmamos desta forma que este algoritmo, o qual gera amostras efetivas não correlacionadas, ganha eficiência no tempo de estimação dos parâmetros quando temos uma amostra com mais de 500 unidades no modelo da TRT 2TP. 


\subsection{Aplicação}

Na presente seção usamos o algoritmo NUTS para ajustar os modelos da teoria de resposta ao testlet (TRT): 2TP, 2TL, 1TP e 1TL em um conjunto de dados reais. O conjunto de dados esta conformado por respostas corretas e incorretas de 730 estudantes, os quais pertencem ao segundo ano do ensino fundamental das escolas do Peru, estes estudantes foram avaliados em Compreensão Leitora (CL) usando como instrumento de medição uma prova com 23 itens, a qual tem um arranjo de 5 testlets. A tabela 2.2 apresenta a estrutura dos testlets da prova, isto é, os grupos de itens os quais compõem um determinado testlet.

Tabela 2.2: Estrutura dos Testlets para o conjunto de dados da Compreensão Leitora

\begin{tabular}{c|c}
\hline testlet & items \\
\hline 1 & $3,4,5,6$ \\
2 & $7,8,9,10$ \\
3 & 12,13 \\
4 & $14,15,16,17,18$ \\
5 & $19,20,21,22,23$ \\
no testlet & $1,2,11$ \\
\hline
\end{tabular}

Para conhecer o desempenho dos estudantes na prova de CL a Figura 2.1 apresenta o histograma dos escores dos estudantes e também a proporção de respostas corretas. O histograma dos escores sugere que a maioria dos estudantes atingem escores acima de 15 pontos e a proporção de respostas corretas revela também o bom desempenho dos estudantes neste conjunto de dados.
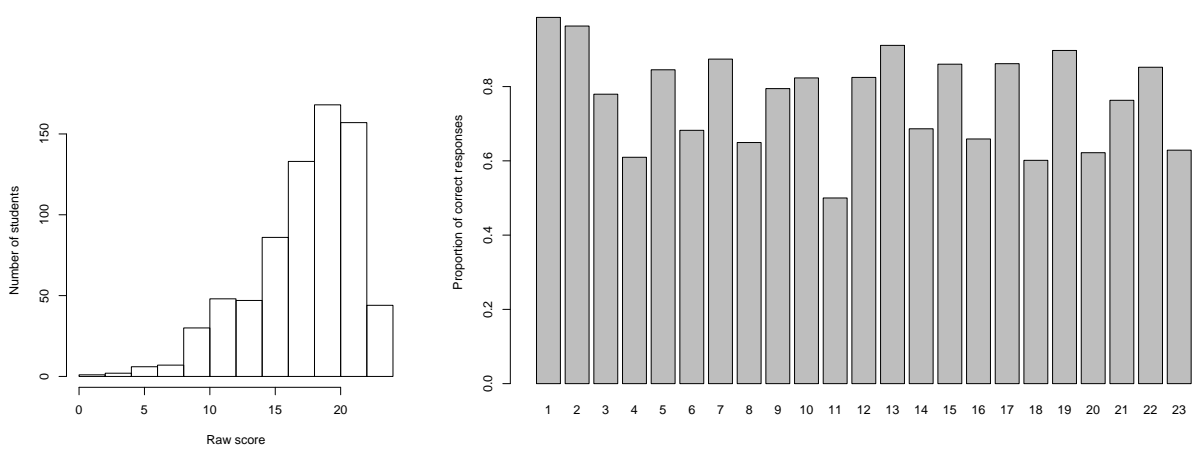

Figura 2.1: (a) histograma dos escores (pontuações totais) e (b) Proporção de respostas corretas

Com o intuito de ilustrar o uso do algoritmo NUTS para os modelos testlets, ajustamos os seguintes modelos da TRT no conjunto de respostas de CL: Modelo testlet probito de dois parâmetros (2TP), modelo testlet probito de um parâmetro (1TP), modelo testlet logito de dois parâmetros (2TL) e o modelo testlet logito de um parâmetro (1TL). Para comparar os modelos ajustados utilizase o critério de informação amplamente aplicável (do acrônimo em inglês WAIC: widely applicable information criterion), o qual avalia a acurácia preditiva para uma estimação pontual distinta à amostra ajustada, veja Vehtari et al. (2017). A Tabela 2.3 apresenta o critério WAIC alcançado em cada modelo. Observa-se que o modelo 2TP tem uma melhor acurácia preditiva para o conjunto de dados da presente aplicação. Em consequência apresentamos na Tabela 2.4 as estimativas para 
cada um dos parâmetros do modelo 2TP.

Tabela 2.3: WAIC para os modelos da TRT ajustados no conjunto de dados da $C L$

\begin{tabular}{l|l}
\hline Modelo ajustado & WAIC \\
\hline modelo 2TP & 14145 \\
modelo 1TP & 14441 \\
modelo 2TL & 14212 \\
modelo 1TL & 14444 \\
\hline
\end{tabular}

Em relação ao critério de convergência na estimação do modelo $2 \mathrm{TP}$ podemos observar a estatística $\hat{R}$ (Rhat) na Tabela 2.4 onde as quantidades de $\hat{R}$ são inferiores de 1.1, este resultado revela uma convergência aceitável na estimação dos parâmetros do modelo 2TP. Podemos observar também as estimativas dos parâmetros para o modelo 2TP, isto é, a estimação da média a posteriori, o desvío padrão da média, o desvío padrão, os quanties, a quantidade de amostra efetiva para cada um dos parâmetros do item, os quais são a discriminação $a_{j}$ e a dificuldade $b_{j}$. Note-se que as estimativas da discriminação $a$ estão ao redor de 1 , entre 0.3 e 1.7 . O parâmetro de dificuldade $b$ estão no intervalo de -3.4 ate 0.03 . Enquanto ao parâmetro do efeito testlet, temos que as estimativas são menores de 0.5 e maiores de zero. Adicionalmente, na figura 2.2 retratamos a média aposteriori dos parâmetros de discriminação em relação à dificuldade para cada um dos items.

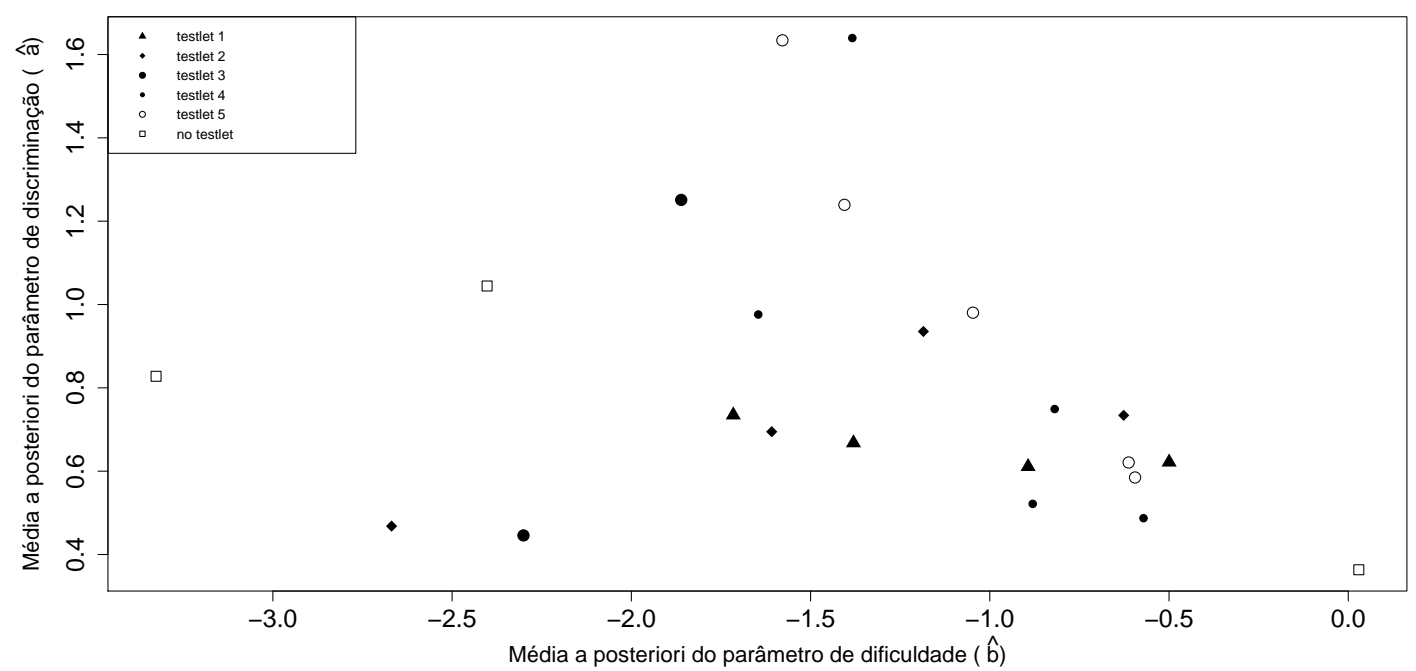

Figura 2.2: Estimativas dos parâmetros de discriminação e dificuldade para o modelo da TRT 2TP aplicado no conjunto de dados da $C L$.

Podemos observar na Figura 2.2 que a média a posteriori da dificuldade dos itens no interior dos testlets 4 e 5 se encontram no lado direito da Figura 2.2, o qual sugere que estes testlets possuem itens com maiores dificuldades, o testlet 3 está no lado esquerdo da Figura o qual sugere que os itens associados no testlet 3 têm menor dificuldade. Assim, podemos observar três grupos diferenciados enquanto à posição da dificuldade dos itens:

- Grupo 1: Itens os quais têm dificuldade menor que -2 ,

- Grupo 2: Itens os quais têm dificuldade maiores que -2 e menores que -1 , 
Tabela 2.4: Estimativas a posteriori do modelo testlet probito de dois parâmetros (2TP)

\begin{tabular}{|c|c|c|c|c|c|c|c|c|c|c|}
\hline Parâmetro & média & d.p. da média & desvío padrao (d.p.) & quantil 0.025 & quantil 0.25 & quantil 0.50 & quantil 0.75 & quantil 0.975 & amostra efetiva & Rhat \\
\hline$a_{1}$ & 0.82773 & 0.00243 & 0.142 & 0.59598 & 0.72521 & 0.81419 & 0.91408 & 1.14669 & 3428 & 0.99967 \\
\hline$a_{2}$ & 1.04444 & 0.00324 & 0.17295 & 0.74483 & 0.92308 & 1.03148 & 1.15077 & 1.42164 & 2851 & 1.00096 \\
\hline$a_{3}$ & 0.66781 & 0.00182 & 0.08454 & 0.50788 & 0.60797 & 0.66558 & 0.72397 & 0.84419 & 2154 & 0.9999 \\
\hline$a_{4}$ & 0.62161 & 0.00153 & 0.07559 & 0.48036 & 0.57091 & 0.61909 & 0.67052 & 0.77689 & 2426 & 1.00059 \\
\hline$a_{5}$ & 0.73468 & 0.002 & 0.09643 & 0.56261 & 0.66742 & 0.72953 & 0.79433 & 0.93746 & 2315 & 1.00018 \\
\hline$a_{6}$ & 0.61098 & 0.00249 & 0.07772 & 0.46492 & 0.55705 & 0.61085 & 0.66087 & 0.77172 & 975 & 1.00654 \\
\hline$a_{7}$ & 0.46813 & 0.00107 & 0.06512 & 0.35098 & 0.42277 & 0.46638 & 0.51115 & 0.60444 & 3676 & 1.00057 \\
\hline$a_{8}$ & 0.73406 & 0.00155 & 0.08458 & 0.57638 & 0.67544 & 0.73028 & 0.78919 & 0.90834 & 2988 & 0.99982 \\
\hline$a_{9}$ & 0.93527 & 0.00227 & 0.11358 & 0.73549 & 0.85515 & 0.92755 & 1.00607 & 1.18311 & 2514 & 0.99978 \\
\hline$a_{10}$ & 0.69472 & 0.00184 & 0.09258 & 0.5267 & 0.62955 & 0.68999 & 0.75646 & 0.89189 & 2531 & 0.99967 \\
\hline$a_{11}$ & 0.36337 & 0.00092 & 0.05792 & 0.25157 & 0.32451 & 0.36334 & 0.40138 & 0.47996 & 4000 & 0.99995 \\
\hline$a_{12}$ & 0.44583 & 0.00194 & 0.06503 & 0.32618 & 0.4006 & 0.44247 & 0.48729 & 0.58199 & 1122 & 1.00404 \\
\hline$a_{13}$ & 1.25107 & 0.03315 & 0.3348 & 0.83341 & 1.04204 & 1.18275 & 1.37041 & 2.17485 & 102 & 1.0192 \\
\hline$a_{14}$ & 0.74914 & 0.00255 & 0.08846 & 0.58658 & 0.68776 & 0.74463 & 0.80716 & 0.93377 & 1205 & 0.99977 \\
\hline$a_{15}$ & 1.63954 & 0.00839 & 0.30439 & 1.15331 & 1.42952 & 1.6004 & 1.80168 & 2.34567 & 1315 & 1.00053 \\
\hline$a_{16}$ & 0.52161 & 0.00161 & 0.06513 & 0.39808 & 0.47658 & 0.5197 & 0.56454 & 0.65446 & 1644 & 1.00007 \\
\hline$a_{17}$ & 0.97602 & 0.0035 & 0.13018 & 0.74788 & 0.88284 & 0.96888 & 1.06002 & 1.24162 & 1380 & 1.00086 \\
\hline$a_{18}$ & 0.4872 & 0.00165 & 0.0619 & 0.37345 & 0.44306 & 0.48387 & 0.52768 & 0.61095 & 1414 & 0.99958 \\
\hline$a_{19}$ & 1.6341 & 0.00773 & 0.29759 & 1.16011 & 1.43223 & 1.59326 & 1.79644 & 2.35881 & 1483 & 1.00081 \\
\hline$a_{20}$ & 0.5849 & 0.00167 & 0.07026 & 0.45256 & 0.53666 & 0.58262 & 0.6311 & 0.73147 & 1763 & 1.00022 \\
\hline$a_{21}$ & 0.98043 & 0.00349 & 0.11779 & 0.76654 & 0.89714 & 0.97474 & 1.05605 & 1.23092 & 1137 & 1.00019 \\
\hline$a_{22}$ & 1.23928 & 0.00358 & 0.16922 & 0.93872 & 1.12103 & 1.22753 & 1.34532 & 1.60851 & 2233 & 1.00177 \\
\hline$a_{23}$ & 0.62088 & 0.0027 & 0.07675 & 0.47979 & 0.56772 & 0.61846 & 0.67076 & 0.77694 & 807 & 1.00269 \\
\hline$b_{1}$ & -3.32586 & 0.00617 & 0.39048 & -4.15083 & -3.57456 & -3.29952 & -3.05072 & -2.63651 & 4000 & 1.00011 \\
\hline$b_{2}$ & -2.40241 & 0.0048 & 0.24992 & -2.9524 & -2.55467 & -2.37882 & -2.22372 & -1.97691 & 2716 & 0.99994 \\
\hline$b_{3}$ & -1.38035 & 0.00407 & 0.16228 & -1.73995 & -1.47601 & -1.36584 & -1.26683 & -1.10074 & 1588 & 1.00111 \\
\hline$b_{4}$ & -0.49978 & 0.00161 & 0.10183 & -0.70873 & -0.56432 & -0.49704 & -0.43053 & -0.30839 & 4000 & 1.0013 \\
\hline$b_{5}$ & -1.71559 & 0.00422 & 0.18387 & -2.10535 & -1.82976 & -1.70279 & -1.58612 & -1.38094 & 1902 & 1.00236 \\
\hline$b_{6}$ & -0.89316 & 0.00354 & 0.13446 & -1.18017 & -0.9776 & -0.8843 & -0.80063 & -0.65681 & 1445 & 1.00457 \\
\hline$b_{7}$ & -2.66879 & 0.00594 & 0.34262 & -3.41942 & -2.88581 & -2.64064 & -2.42593 & -2.08401 & 3332 & 1.00109 \\
\hline$b_{8}$ & -0.62655 & 0.00153 & 0.09677 & -0.82723 & -0.6881 & -0.61998 & -0.56091 & -0.45125 & 4000 & 1.00051 \\
\hline$b_{9}$ & -1.18532 & 0.00279 & 0.1161 & -1.43568 & -1.2604 & -1.17888 & -1.10557 & -0.97502 & 1736 & 1.00063 \\
\hline$b_{10}$ & -1.60838 & 0.00418 & 0.18862 & -2.02476 & -1.72149 & -1.59219 & -1.47423 & -1.28912 & 2036 & 1.00053 \\
\hline$b_{11}$ & 0.02937 & 0.00217 & 0.13726 & -0.23909 & -0.06145 & 0.02971 & 0.11721 & 0.30159 & 4000 & 1.00033 \\
\hline$b_{12}$ & -2.30093 & 0.01985 & 0.31929 & -3.01528 & -2.49477 & -2.27374 & -2.07818 & -1.74491 & 259 & 1.00832 \\
\hline$b_{13}$ & -1.86085 & 0.01542 & 0.18339 & -2.27242 & -1.96525 & -1.84371 & -1.73332 & -1.55271 & 141 & 1.0108 \\
\hline$b_{14}$ & -0.81907 & 0.00337 & 0.11071 & -1.05325 & -0.89211 & -0.81199 & -0.74079 & -0.61936 & 1081 & 1.00009 \\
\hline$b_{15}$ & -1.38352 & 0.00536 & 0.11676 & -1.62459 & -1.45605 & -1.37663 & -1.3044 & -1.17508 & 474 & 1.00087 \\
\hline$b_{16}$ & -0.88034 & 0.00317 & 0.14421 & -1.18595 & -0.96927 & -0.87034 & -0.78159 & -0.61936 & 2063 & 1.00077 \\
\hline$b_{17}$ & -1.64592 & 0.0058 & 0.15372 & -1.97149 & -1.74349 & -1.6392 & -1.53561 & -1.37771 & 702 & 1.00068 \\
\hline$b_{18}$ & -0.57125 & 0.00201 & 0.12699 & -0.83314 & -0.65253 & -0.56588 & -0.48141 & -0.34794 & 4000 & 0.99956 \\
\hline$b_{19}$ & -1.57859 & 0.00668 & 0.12704 & -1.84525 & -1.65906 & -1.57354 & -1.49211 & -1.34844 & 361 & 1.00424 \\
\hline$b_{20}$ & -0.59479 & 0.00182 & 0.11498 & -0.84544 & -0.66689 & -0.59049 & -0.51558 & -0.38716 & 4000 & 0.9998 \\
\hline$b_{21}$ & -1.04701 & 0.0043 & 0.10742 & -1.26624 & -1.11569 & -1.04249 & -0.97057 & -0.85329 & 624 & 1.00046 \\
\hline$b_{22}$ & -1.40556 & 0.00496 & 0.1197 & -1.65251 & -1.48196 & -1.40413 & -1.32322 & -1.18877 & 583 & 1.00272 \\
\hline$b_{23}$ & -0.61262 & 0.00178 & 0.11288 & -0.84628 & -0.68533 & -0.60556 & -0.53625 & -0.40181 & 4000 & 0.99984 \\
\hline$\sigma_{1}^{2}$ & 0.16036 & 0.01317 & 0.08304 & 0.0331 & 0.09838 & 0.15305 & 0.20971 & 0.34684 & 40 & 1.0921 \\
\hline$\sigma_{2}^{2}$ & 0.12422 & 0.00815 & 0.0481 & 0.04958 & 0.08839 & 0.11982 & 0.15253 & 0.23318 & 35 & 1.09835 \\
\hline$\sigma_{3}^{2}$ & 0.36176 & 0.0548 & 0.25288 & 0.06148 & 0.17325 & 0.29352 & 0.48327 & 0.97419 & 21 & 1.08068 \\
\hline$\sigma_{4}^{2}$ & 0.45065 & 0.00889 & 0.11591 & 0.24021 & 0.37329 & 0.44176 & 0.52118 & 0.69686 & 170 & 1.00309 \\
\hline$\sigma_{5}^{2}$ & 0.33661 & 0.00919 & 0.09516 & 0.1801 & 0.26766 & 0.32686 & 0.3918 & 0.5583 & 107 & 1.01822 \\
\hline
\end{tabular}

- Grupo 3: Itens os quais têm posição maior que -1 .

Estes grupos sugerem que a prova esta pensada na medição de três conjuntos diferenciados de itens. Podemos notar também que a estimação do parâmetro de discriminação, o qual pode ser interpretado como o inverso da variância associada às respostas dos itens, têm quantidades diferentes em cada um destes grupos formados. No grupo 3, onde se encontram os itens com maiores dificuldades, a discriminação dos itens é pequena, o qual sugere que a probabilidade de acertar o item não é muito distante para habilidades diferentes do individuo. Observamos também existência de dependência no interior dos testlets $\left(\sigma_{\gamma_{l}}^{2}>0\right)$ com maior dependência no testlet 4 . Isto é, existência de variabilidade dos efeitos testlet $\gamma_{i 4}$ para os indivíduos avaliados. O qual sugere que o fato de ter itens agrupados no testlet 4 tem como consequência um efeito diferenciado nos indivíduos avaliados.

\subsection{Comentários finais}

No presente capítulo, revisamos os modelos da teoria de resposta ao testlet (TRT), considerando distinções enquanto às funções de ligação probito e logito e em relação a quantidade dos parâmetros 
do item. Implementamos o uso do algoritmo Hamiltoniano Monte Carlo com a adaptação NUTS e fizemos uma comparação com o algoritmo de Metropolis Hastings. O estudo de simulação mostrou melhor desempenho da adaptação NUTS do algoritmo Hamiltoniano Monte Carlo em relação ao algoritmo de Metropolis Hastings quando o tamanho da amostra é maior de 500 unidades. Isto é, maior amostra efetiva é obtida com o algoritmo Hamiltoniano NUTS e ademais menor tempo computacional na estimação dos parâmetros. Quando temos amostras de 1000 unidades o tempo no algoritmo Hamiltoniano é menos da metade do tempo que usa o algoritmo do Metropolis Hastings. Uma aplicação ao conjunto de dados de compreensão leitora foi desenvolvida, usando o algoritmo Hamiltoniano NUTS para quatro modelos da TRT, onde o modelo testlet probito de dois parâmetros apresentou um melhor desempenho na preditiva a posteriori, segundo o critério WAIC. O resultado da aplicação sugere a existência de variabilidade nos efeitos testlet $\left(\sigma_{\gamma_{l}}^{2}>0\right)$, isto é, da conta da existência de dependência no interior dos testlets para o conjunto de dados analisado. 


\section{Capítulo 3}

\section{Modelos de regressão espacial com resposta limitada para o estudo da compreensão leitora}

\subsection{Introdução}

Recentemente na literatura foram propostos modelos de regressão para variáveis de resposta limitada, estes modelos consideram diferentes distribuições para modelar a variável resposta, por exemplo, a distribuição Beta Cepeda-Cuervo e Núñez-Antón (2013); Ferrari e Cribari-Neto (2004); Kieschnick e McCullough (2003), a Simplex Song et al. (2004); Song e Tan (2000), a Kumaraswamy Bayes et al. (2017); Bonat et al. (2013), a L-Logistic da Paz et al. (2018), de forma mais geral a distribuição CDF-quantile Smithson e Shou (2017) e o sistema generalizado Johnson Lemonte e Bazán (2016).

$\mathrm{Na}$ análise de regressão, quando assume-se que a resposta segue uma distribuição Beta ou Simplex, a média e a dispersão estão associadas com um conjunto de covariáveis, as quais consideram funções de ligação adequadas. No entanto, as distribuições Kumaraswamy, CDF-quantile, GJS e L-Logistic usam o quantile, de preferência a mediana, e o parâmetro de dispersão para introduzir as covariáveis. Adicionalmente, modelos de regressão mistos foram apresentados na literatura, os quais consideram a distribuição Beta, Simplex e Kumaraswamy. Alguns deles foram desenvolvidos pelos autores: Verkuilen e Smithson (2012), Figueroa-Zuñiga et al. (2013), Qiu et al. (2008) and Bayes et al. (2017). Os modelos mistos são convenientes quando as observações nos dados são medidas, por exemplo, de forma repetida no tempo, ou quando existem unidades agrupadas em clusters. Para estes conjuntos de dados é necessário incluir heterogeneidade entre unidades usando efeitos aleatórios ou estruturas hierárquicas ou multiníveis.

No entanto, de forma frequente encontram-se conjuntos de dados onde é comum a dependência espacial entre unidades. Por exemplo, a proporção de estudantes com nível satisfatório em compreensão leitora para cidades de um país ou a proporção de pessoas em situação de pobreza em aquelas cidades. Nestes ambos os casos são possíveis considerar que as características comuns entre cidades próximas, as quais compartilham idiossincrasias mútuas, estão associadas à variabilidade 
observada nas unidades. Assim, uma configuração espacial apropriada a qual é baseada na vizinhança deveria ser incorporada na análise de regressão com o propósito de sugerir modelos mais plausíveis.

De momento, a literatura apresenta só um modelo de regressão espacial para proporções, o qual assume que a variável resposta segue uma distribuição Beta. Especificamente, Cepeda-Cuervo e Núñez-Antón (2013) sugerem o modelo de regressão beta doblemente generalizado. O qual usa uma aproximação de modelamento conjunta para os parâmetros da média e a dispersão e foi aplicado na análise da qualidade da educação em Colômbia. No entanto, vários autores têm comprovado que nem sempre é apropriada a suposição de que a variável resposta assuma uma distribuição Beta, por exemplo, a Simplex López (2013) e a Kumaraswamy Bayes et al. (2017) são boas alternativas para o modelamento das respostas. Além disso, na literatura não são propostos modelos espaciais usando a distribuição Simplex e Kumaraswamy, no contexto da inferência Bayesiana.

Assim, no presente capítulo é proposto um modelo de regressão espacial usando a estimação Bayesiana para variáveis de resposta limitada. A formulação do modelo proposto considera que a variável resposta segue alguma distribuição limitada no intervalo unitário $(0,1)$, como por exemplo a Simplex ou a Kumaraswamy, e que as relações de dependência entre vizinhos da variável resposta são modeladas usando o modelo autorregressivo condicional intrínseco (ICAR) Besag e Kooperberg (1995); Besag et al. (1991) ou a definição de Leroux Leroux et al. (2000) com o intuito de modelar as relações espaciais nos dados. Adicionalmente, possíveis diferenças em comparação com o modelo de regressão espacial Beta podem ser identificadas.

O presente capítulo é organizado em diferentes seções. Na seção 3.2, como motivação, apresentase um conjunto de dados e uma análise preliminar. Na seção 3.3, define-se três distribuições limitadas com o intuito de modelar a variável resposta, as quais tem suporte limitado no intervalo unitário. Na seção 3.4, propõe-se o modelo geral de regressão espacial para respostas limitadas, o qual considera as distribuições apresentadas na seção previa e o efeito aleatório espacial, com a intenção de modelar a dependência espacial. Na Seção 3.5, uma aproximação Bayesiana é formulada e desenvolvida, para os modelos de regressão propostos, usando critérios de comparação de modelos com a finalidade de ter uma escolha adequada entre modelos alternativos. A Secão 3.6 apresenta resultados dos estudos de simulação desenvolvidos os quais revelam o desempenho na recuperação dos parâmetros e os critérios de comparação de modelos. Para aplicar a metodologia proposta o conjunto de dados é analisado novamente na Seção 3.7 usando os modelos de regressão espaciais sugeridos. Comentários adicionais e desenvolvimentos futuros são apresentados na Seção 3.8.

\subsection{O conjunto de dados e a análise preliminar}

Desde o ano 2006, anualmente acontece a Avaliação Nacional de Estudantes (com ECE como acrônimo no espanhol) a qual é realizada pelo Ministério de Educação do Peru. A ECE tem a intenção de conhecer o nível de sucesso dos estudantes em Compreensão Leitora (CL) e Matemática para o segundo ano do ensino fundamental. No 2012, a ECE atingiu uma taxa de cobertura de escolas de $97.7 \%$, em escolas com cinco o mais estudantes, e uma cobertura de estudantes de $89.4 \%$. Os 


\begin{tabular}{lrrrr}
\hline Estadísticas descriptivas & $y$ & $x_{1}$ & $x_{2}$ & $x_{3}$ \\
\hline Mínimo & 0.023 & 0.043 & 0.002 & 0.338 \\
Quartil 1 & 0.099 & 0.108 & 0.410 & 0.613 \\
Mediana & 0.167 & 0.173 & 0.542 & 0.743 \\
Média & 0.203 & 0.199 & 0.563 & 0.721 \\
Quartil 3 & 0.284 & 0.246 & 0.725 & 0.850 \\
Máximo & 0.616 & 0.746 & 0.995 & 0.995 \\
Variância & 0.017 & 0.015 & 0.042 & 0.025 \\
Assimetria & 0.919 & 1.595 & 0.068 & -0.29 \\
\hline
\end{tabular}

Tabela 3.1: Estadísticas Descriptivas para a variável resposta e as covariáveis do conjunto de dados CL.

resultados estão disponíveis no portal institucional do ministério, accessível a través do seguinte link http://umc.minedu.gob.pe/evaluacion-censal-de-estudiantes-2012-ece-2012/.

O propósito neste trabalho é explicar a variável resposta $y$ : Proporção de estudantes com nível satisfatório em Compreensão Leitora (CL) Unidad de Medición de la Calidad Educativa (2012) para cada uma das 195 províncias peruanas (divisão política de segundo nível) durante o ano 2012. Detalhes adicionais em relação à prova de CL são apresentados no documento do Ministério MINEDU (2016). Como covariáveis foram consideradas alguns índices os quais são componentes do índice de densidade do estado (IDE) PNUD (2013). O índice IDE avalia a provisão de serviços essenciais para construir o desenvolvimento humano. Especificamente, o trabalho considera o índice de saúde, o qual quantifica o número de médicos para 10,000 habitantes, com valores entre 0 e 60; quantidades maiores indicam a existência de maiores médicos e, consequentemente, maior acesso à saúde. Considera-se também o índice de saneamento, o qual quantifica a percentagem de imóveis com água e drenagem, este índice tem valores na faixa de 0 a 100. Finalmente, considera-se o índice de eletrificação, isto é a percentagem de prédios com eletricidade, o qual tem uma faixa de 0 a 100. Detalhes em relação ao computo dos indicadores podem ser encontrados no documento PNUD (2013) e o conjunto de dados estão disponíveis no site http://www.pe.undp.org/content/ peru/es/home/library/poverty/Informesobredesarrollohumano2013/IDHPeru2013.html. Com o intuito de formular um modelo de regressão, as covariáveis saúde, saneamento e eletrificação foram transformadas para uma escala de proporção no intervalo unitário $(0,1)$ e nomeado como $x_{1}, x_{2}$ e $x_{3}$ respectivamente. Algumas estadísticas descritivas para a variável resposta e as covariáveis são apresentadas na tabela 3.1.

Como a variável resposta é definida no intervalo $(0,1)$, uma primeira opção natural para ajustar o conjunto de dados é o seguinte modelo de regressão Beta:

$$
\begin{gathered}
y_{i} \mid \boldsymbol{\beta}, \phi \stackrel{i n d .}{\sim} \operatorname{Beta}\left(\mu_{i}, \phi\right) \\
\log \left(\frac{\mu_{i}}{1-\mu_{i}}\right)=\beta_{0}+\beta_{1} x_{1 i}+\beta_{2} x_{2 i}+\beta_{3} x_{3 i} \\
i=1, \ldots, 195
\end{gathered}
$$

onde $\mu_{i}$ e $\phi$ são os parâmetros, média e precisão respectivamente, da distribuição Beta. $\beta_{0}$ é o intercepto e $\beta_{m}, m=1,2,3$ são os coeficientes de regressão associados com as covariáveis. Considerando a inferência Bayesiana, os parâmetros do modelo foram estimados usando o método de aproximação integrada e aninhada de Laplace (com INLA como acrônimo em inglês) o qual é implementado no pacote R-INLA (Rue et al. (2009), http://www.r-inla.org). Distribuições a priori não informativas predefinidas (veja Blangiardo e Cameletti (2015), cap. 5) são usadas para o modelo completo, tais 


\begin{tabular}{lrrr}
\hline Parâmetro & Média & Desvio padrão & Intervalo de credibilidade de 95\% \\
\hline$\beta_{0}$ & -3.63 & 0.19 & $(-4.01,-3.26)$ \\
$\beta_{1}$ & 1.79 & 0.36 & $(1.08,2.50)$ \\
$\beta_{2}$ & 1.27 & 0.28 & $(0.73,1.82)$ \\
$\beta_{3}$ & 1.51 & 0.33 & $(0.86,2.17)$ \\
$\phi$ & 24.79 & 2.47 & $(20.21,29.89)$ \\
\hline
\end{tabular}

Tabela 3.2: Estimativas dos parâmetros no modelo de regressão Beta para os dados de Compreensão Leitora

prioris são:

$$
\begin{gathered}
\log (\phi) \sim \log \operatorname{Gamma}(1,0.1) \\
\beta_{m} \sim \mathcal{N}\left(0,10^{6}\right), \quad m=0,1,2,3 .
\end{gathered}
$$

A Tabela 3.2 visualiza a média a posteriori, o desvio padrão e o intervalo de credibilidade (IC) de $95 \%$ para os parâmetros do modelo de regressão ajustado. Olhando o IC dos coeficientes de regressão observa-se que o IC não inclui o zero, então é possível concluir que as covariáveis são significativas e fornecem um efeito positivo na variável resposta. Em outras palavras, se os serviços essenciais em uma província incrementam a proporção de estudantes com nível satisfatório em CL também melhoram.

Com a finalidade de avaliar o ajuste do modelo, inicialmente, foram calculados os resíduos padronizados. Tais resíduos são definidos a seguir: $r_{i}=\left(y_{i}^{\star}-y_{i}\right) / \sqrt{\operatorname{vâr}\left(y_{i}^{\star}\right)}$, onde $y_{i}^{\star}$ é a média a posteriori da distribuição preditiva dos parâmetros, veja Blangiardo e Cameletti (2015) seção 5.5 e 5.6. A estimativa posterior da variância é definida como vâr $\left(y_{i}^{\star}\right)=y_{i}^{\star}\left(1-y_{i}^{\star}\right) /(1+\hat{\phi})$, onde $\hat{\phi}$ é a média da distribuição a posteriori do parâmetro $\phi$. Os códigos no cálculo dos resíduos são incluídos no anexo.

O lado esquerdo da Figura E.1 ilustra, para cada unidade $i$, o resíduo padronizado versus a média posterior da distribuição preditiva $y_{i}^{\star}$. Uma faixa no eixo das ordenadas foi incluída para os pontos $\Phi^{-1}(.025)=-1.96$ e $\Phi^{-1}(.975)=1.96$. Onde $\Phi($.$) denota a função de distribuição acumu-$ lada (fda) da distribuição normal. Na Figura E.1 observa-se alguns resíduos estão fora da faixa, isto é um indicativo que o modelo não tem um bom ajuste nos dados, pois observa-se resíduos maiores. O modelo ajustado induz uma distribuição de dados futuros a qual é condicionada nas observações, esta distribuição é chamada a distribuição preditiva a posteriori (PPD). A PPD é utilizada para gerar conjuntos de dados sintéticos. Considerado valores de $\hat{\mu}_{i}$ e $\hat{\phi}, i=1, \ldots, n, M=1000$ conjuntos de dados de tamanho $n$ foram gerados usando a distribuição preditiva de $y_{i}$. Com o intuito de obter a faixa de confiança da PPD, o conjunto de dados gerado para cada réplica foi agrupado em tabelas de frequência com intervalos de tamanho 0.03. Os quantiles 0.05 e 0.95 do conjunto de frequências geradas são apresentadas como linhas tracejadas e o quantile 0.5 como uma linha contínua, no lado dereito da Figura E.1, junto com o histograma da variável resposta observada. Podemos observar que o tamanho da faixa de confiança é maior para valores da resposta em torno do 0.15 e algumas observações estão fora da faixa, o qual sugere que o modelo não tem um bom ajuste para o conjunto de dados analisado. Então, é possível concluir que ambos os resultados apresentados na Figura E.1 sugerem ausência de ajuste no modelo de regressão Beta.

Adicionalmente, com a intenção de identificar uma possível configuração espacial da variável 

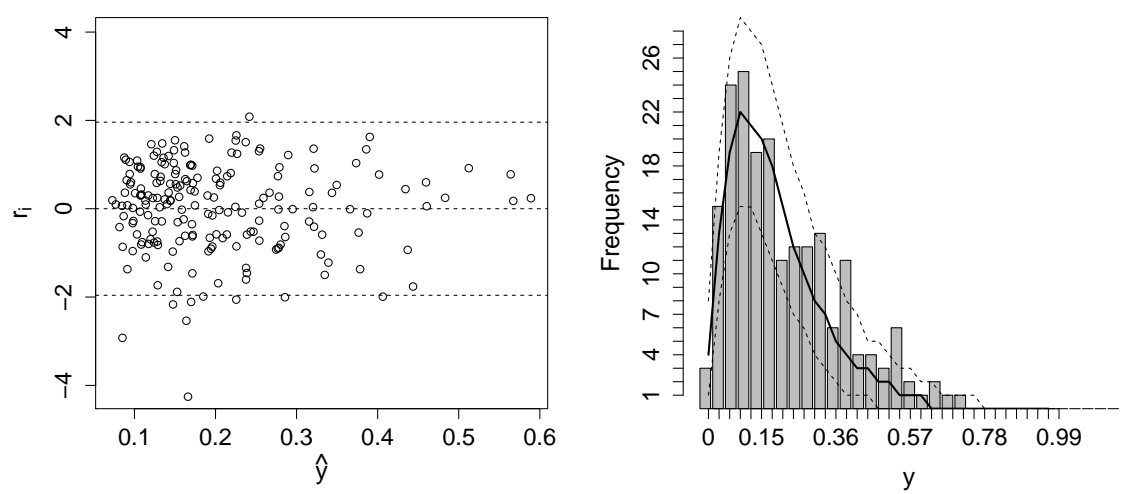

Figura 3.1: Ajuste do modelo de regressão Beta: (a) resíduo padronizado $r_{i}$ versus a variável resposta estimada $\hat{y}_{i}$, (b) faixa de confiança da densidade preditiva a posteriori com o histograma do conjunto de dados $C L$.

resposta, a Figura E.2 retrata os resíduos do modelo ajustado para cada uma das províncias. Tais resíduos são categorizados em intervalos de tamanho 0.91, onde cada intervalo tem um nível de cor específico. As províncias com uma tonalidade clara de cor indicam resíduos negativos e uma cor escura indica que a província tem um resíduo positivo. Assim, de forma espacial, é possível observar presença de agrupamento de províncias as quais tem um comportamento similar não explicado no modelo. Alguns destes resíduos são positivos, outros negativos e também têm resíduos pequenhos.

Em efeito, identifica-se uma configuração espacial para as províncias a qual precisa uma análise maior com o intuito de formular um modelo apropriado para o conjunto de dados de CL. Esta configuração espacial não foi incluída no modelo de regressão Beta analisado. O resultado foi também observado considerando os modelos de regressão alternativos sem o efeito espacial assumindo a distribuição Simplex ou Kumaraswamy o qual é apresentado na Seção 3.7. Assim, o conjunto de dados de CL motiva a formulação do modelo de regressão espacial para proporções o qual pode ser utilizado como um modelo alternativo com a intenção de conhecer relações entre cidades vizinhas.

Nesse sentido, o presente capítulo introduz modelos espaciais para variáveis de resposta limitada utilizando outras distribuições além da Beta, a qual foi utilizada na proposta desenvolvida pelos autores Cepeda-Cuervo e Núñez-Antón (2013), e inclui duas formas de considerar os efeitos aleatórios espaciais.

\subsection{Variáveis de resposta limitada}

Nesta seção define-se três distribuições para as variáveis de resposta limitada as quais têm suporte no intervalo $(0,1)$.

- Uma variável aleatória $Y$ segue uma distribuição Beta com parâmetros $\mu$ e $\phi_{1}$ se a função de densidade de probabilidade (fdp) é definida como a seguir:

$$
g\left(y \mid \mu, \phi_{1}\right)=\frac{\Gamma\left(\phi_{1}\right)}{\Gamma\left(\mu \phi_{1}\right) \Gamma\left[(1-\mu) \phi_{1}\right]} y^{\mu \phi_{1}-1}(1-y)^{(1-\mu) \phi_{1}-1} I_{(0,1)}(y) .
$$




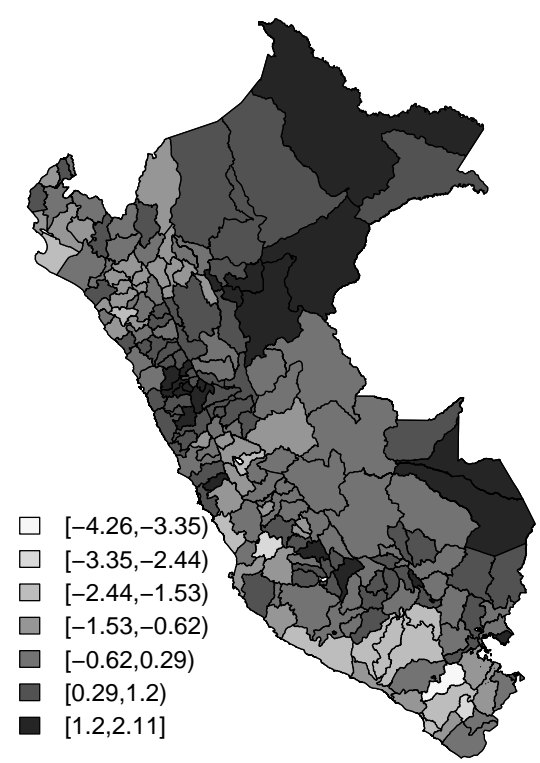

Figura 3.2: Mapa das 195 províncias peruanas junto com os resíduos, comportamento não explicado, do modelo de regressão Beta

A notação utilizada é $Y \sim \operatorname{Beta}\left(\mu, \phi_{1}\right)$ com $\mu \in(0,1)$ sendo o parâmetro de posição e $\phi_{1}>0$ o parâmetro de precisão. O valor esperado e a variância da distribuição Beta são respectivamente:

$$
\begin{aligned}
& E\left[Y \mid \mu, \phi_{1}\right]=\mu \mathrm{e} \\
& \operatorname{Var}\left[Y \mid \mu, \phi_{1}\right]=\frac{\mu(1-\mu)}{1+\phi_{1}} .
\end{aligned}
$$

- Uma variável aleatória $Y$ segue uma distribuição Simplex com parâmetros $\mu$ e $\phi_{2}$ se a fdp da distribuição é definida como a seguir:

$$
g\left(y \mid \mu, \phi_{2}\right)=\frac{\sqrt{\phi_{2}}}{\sqrt{2 \pi\{y(1-y)\}^{3}}} \exp \left[-\frac{\phi_{2}}{2} d(y \mid \mu)\right] I_{(0,1)}(y)
$$

onde $d(y \mid \mu)=\frac{(y-\mu)^{2}}{y(1-y) \mu^{2}(1-\mu)^{2}}$ é o desvio unitário, o qual é positivo e tem o valor zero quando $y=\mu$.

Notação: $Y \sim \mathrm{S}\left(\mu, \phi_{2}\right)$ com $\mu \in(0,1)$ como o parâmetro de posição e $\phi_{2}>0$ como o parâmetro de precisão. Segundo JØrgensen (1997)(pg. 199), o valor esperado e a variância da distribuição Simplex são respectivamente:

$$
\begin{aligned}
E\left(Y \mid \mu, \phi_{2}\right)= & \mu \quad \mathrm{e} \\
\operatorname{Var}\left(Y \mid \mu, \phi_{2}\right)= & \mu(1-\mu)-\sqrt{\frac{\phi_{2}}{2}} \\
& \exp \left\{\frac{\phi_{2}}{2 \mu^{2}(1-\mu)^{2}}\right\} \\
& \Gamma\left(\frac{1}{2}, \frac{\phi_{2}}{2 \mu^{2}(1-\mu)^{2}}\right),
\end{aligned}
$$


onde $\Gamma(a, x)=\int_{x}^{\infty} t^{a-1} e^{-t} d t$, o qual define a funçaõ gamma incompleta.

- Uma variável aleatória $Y$ segue uma distribuição Kumaraswamy se a fdp da variável e definida da seguinte forma:

$$
\begin{aligned}
g\left(y \mid \kappa, \phi_{3}\right)= & -\frac{\log (1-q) \phi_{3}}{\log \left(1-e^{-\phi_{3}}\right) \log (\kappa)} y^{-\frac{\phi_{3}}{\log (\kappa)}-1} \\
& \times\left\{1-y^{-\frac{\phi_{3}}{\log (\kappa)}}\right\}^{\frac{\log (1-q)}{\log \left(1-e^{-\phi_{3}}\right)}-1}
\end{aligned}
$$

A notação definida para esta distribuição é $Y \sim \mathrm{K}\left(\kappa, \phi_{3}, q\right)$ com o parâmetro quantile $\kappa \in$ $(0,1)$ sendo o parâmetro de posição e $\phi_{3}>0$ o parâmetro de precisão. $\kappa=G^{-1}(q)$ onde a probabilidade $q$ é assumida fixa de acordo com o quantile de interesse, e $G^{-1}($.) denota o inverso da fda da distribuição Kumaraswamy.

Sob a parametrização utilizando $\kappa$ e $\phi_{3}$, o valor esperado e a variância da distribuição são definidas a seguir:

$$
\begin{aligned}
& E\left(Y \mid \kappa, \phi_{3}\right)=\frac{\log (1-q)}{\log \left(1-e^{-\phi_{3}}\right)} B\left(1-\frac{\log \kappa}{\phi_{3}}, \frac{\log (1-q)}{\log \left(1-e^{-\phi_{3}}\right)}\right) \\
& \mathrm{e} \\
& \operatorname{Var}\left(Y \mid \kappa, \phi_{3}\right)=\frac{\log (1-q)}{\log \left(1-e^{-\phi_{3}}\right)} B\left(1-\frac{2 \log \kappa}{\phi_{3}}, \frac{\log (1-q)}{\log \left(1-e^{-\phi_{3}}\right)}\right) \\
& -\left(E\left(Y \mid \kappa, \phi_{3}\right)\right)^{2}
\end{aligned}
$$

onde $B(\cdot, \cdot)$ denota a função beta e $\kappa$ denota o parâmetro quantile, o qual é fixo e pode ser definido como a mediana se $q=0.5$.

Em diante considera-se uma variável aleatória $Y$ a qual segue uma distribuição limitada, com notação $Y \sim \pi(\theta, \phi)$, onde $\theta$ é o parâmetro de locação o posição (sendo $\mu$ no caso das distribuições Beta e Simplex ou $\kappa$ em relação à mediana na distribuição Kumaraswamy) e $\phi$ é o parâmetro de precisão sendo $\phi_{1}, \phi_{2}$ ou $\phi_{3}$, os quais foram descritos previamente.

A Figura 3.3 apresenta funções de densidade de probabilidade (fdp) da distribuição limitada. As quais são definidas como a distribuição Beta, Simplex ou Kumaraswamy, com distintos valores para o parâmetro de posição $\theta$ e o parâmetro de precisão $\phi$. No lado esquerdo da Figura 3.3, são apresentados distintos níveis de precisão considerando um valor fixo do parâmetro de locação $\theta=0.5$. No lado direito, a figura ilustra a fdp para distintos valores do parâmetro de posição e um valor fixo do parâmetro de precisão $\phi=\phi_{1}=\phi_{2}=\phi_{3}=1$. Quando o $\theta$ é fixo, observa-se que valores maiores de precisão têm quantidades da variável concentradas ao redor do parâmetro de posição. Quando o parâmetro $\phi$ é fixo, a massa da distribuição muda à direita ou esquerda de acordo com o valor do parâmetro de posição ou locação. Algumas formas adicionais da distribuição limitada são incluídas nas figuras 1, 2 e 3 do apêndice B. Tais formas sugerem um comportamento reflexo em torno do 0.5 para as distribuições Beta e Simplex, porém esta característica não esta presente na distribuição Kumaraswamy. 
Além disso, na Figura 3.4, são apresentados os traços do desvio padrão (sd) das distribuições limitadas fixando o parâmetro de posição $\theta=0.5$ sendo o traço uma função de $\phi$. Na Figura 3.4 observa-se que desvio padrão decresce quando $\phi$ incrementa. Também, observa-se para uma quantidade fixa de $\theta$ e $\phi$, a distribuição Beta tem o maior valor do desvio padrão $s d$, a distribuição Simplex tem o menor valor quando $\phi<10$ e a distribuição Kumaraswamy contém o menor valor para $\phi>10$.

\subsection{O modelo de regressão espacial para respostas limitadas}

Na presente seção formula-se o modelo de regressão espacial para respostas limitadas (SBDR), com o intuito de estudar, além dos efeitos das covariáveis, de que maneira as correlações espaciais entre as províncias peruanas podem explicar a proporção de estudantes com nível satisfatório em Compreensão Leitora (CL).

Com o intuito de definir o modelo SBDR considera-se $n$ variáveis observadas $\boldsymbol{y}=\left\{y_{i}: i=\right.$ $1, \ldots, n\}$ onde $y_{i}$ segue uma distribuição limitada com suporte no intervalo $(0,1)$ como foi definida na Seção 3.3 sendo os parâmetros de posição e precisão $\theta_{i}$ e $\phi$, respectivamente. Ademais $i$ simboliza uma região com uma localização no espaço (coordenadas de latitude e longitude). Também considera-se um conjunto de $p<n$ variáveis explicativas, $\boldsymbol{x}_{i}=\left(x_{i 1}, \ldots, x_{i p}\right)$. Assim, o modelo SBDR é definido como a seguir:

$$
\begin{gathered}
y_{i} \mid z_{i}, \boldsymbol{\beta}, \phi \stackrel{i n d .}{\sim} \pi\left(\theta_{i}, \phi\right) \\
g\left(\theta_{i}\right)=\boldsymbol{x}_{\boldsymbol{i}}^{\top} \boldsymbol{\beta}+z_{i} \\
\boldsymbol{z} \mid \tau \sim \mathcal{N}_{n}(\mathbf{0}, \boldsymbol{R}) \\
i=1, \ldots, n,
\end{gathered}
$$

onde $\pi\left(\theta_{i}, \phi\right)$ denota a distribuição limitada da variável resposta, a qual pode ser a distribuição Beta, Simplex ou Kumaraswamy $\operatorname{com} \theta_{i}$ definida como o parâmetro de posição para o local $i$ (a média para a distribuição Beta e Simplex ou a mediana para a distribuição Kumaraswamy) e $\phi$ é o parâmetro de precisão. A função de ligação $g($.) é assumida como sendo a função logit, mas outras funções de ligação podem ser consideradas. Os parâmetros de regressão desconhecidos ou coeficientes de regressão ou efeitos fixos são simbolizados a través do vetor $\boldsymbol{\beta}=\left(\beta_{0}, \ldots, \beta_{p}\right)^{\top} \in$ $\mathbb{R}^{p+1}, \boldsymbol{X}$ é uma matriz com dimensão $n \times(p+1)$, assume-se que a matriz é de posto completo, a qual contém informação das covariáveis associadas aos efeitos fixos incluindo a intercepção, $\boldsymbol{x}_{\boldsymbol{i}}^{\top}=$ $\left(x_{i 0}, x_{i 1}, \ldots, x_{i p}\right)$ é a fila $i$ de $\boldsymbol{X}$ e $\boldsymbol{R}$ é uma matriz de precisão adequada.

$\mathrm{O}$ vetor dos efeitos aleatórios associados com as localizações $\boldsymbol{z}=\left(z_{1}, \cdots, z_{n}\right)^{\top}$ abrange a dependência espacial. Assume-se que o $z_{i}$ esta distribuída condicionalmente de tal forma que $z_{i} \mid \boldsymbol{z}_{-i}$ denota a distribuição condicional de $z_{i}$ dado todos os outros valores de $\boldsymbol{z}, n_{i}$ é a quantidade de vizinhos da região $i$. Com o intuito de atribuir uma associação espacial, define-se $z_{i} \mid \boldsymbol{z}_{-i}$ como a propriedade de Markov de tal forma que $z_{i}\left|\boldsymbol{z}_{-i}=z_{i}\right| \boldsymbol{z}_{i \sim j}$ onde $i \sim j$ denota os vizinhos da região $i$. Este modelo é conhecido como o modelo condicional autoregressivo intrínseco (ICAR) (Besag et al., 1991). Adicionalmente, $\tau$ define um parâmetro de precisão espacial (o inverso da variância) do $\boldsymbol{z}$. Em outras palavras, $\boldsymbol{z}$ contém as relações entre a região $i$ com os vizinhos $j^{\prime} s$ considerando a 

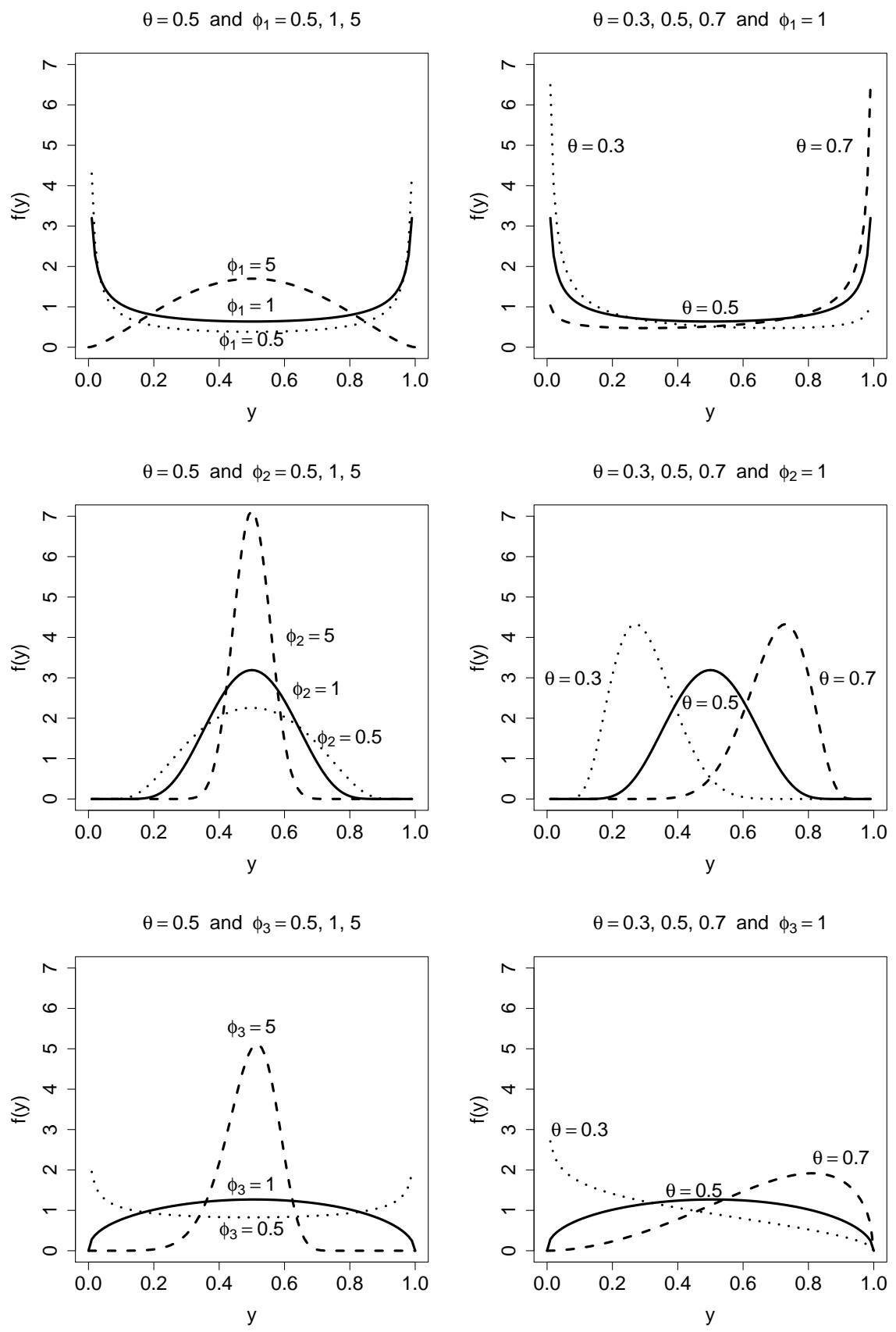

Figura 3.3: Função de densidade de probabilidade da distribução limitada (beta, simplex e kumaraswamy) para distintos valores do parâmetro de posição $\theta$ e precisão $\phi$. 


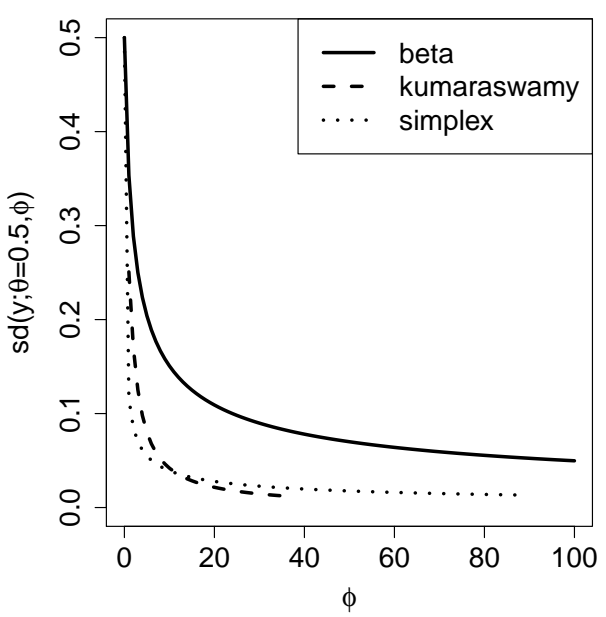

Figura 3.4: Desvío padrão (sd) de uma variável com distribuição limitada como uma função do parâmetro de precisão $\phi$ e para uma quantidade fixa do parâmetro de locação $\theta$

distribuição conjunta como a seguir:

$$
\pi(\boldsymbol{z} \mid \tau) \propto \tau^{r(\boldsymbol{R}) / 2} \exp \left(-\frac{\tau}{2} \boldsymbol{z}^{\top} \boldsymbol{R} \boldsymbol{z}\right)
$$

onde $r($.$) define o posto de uma matriz. A matriz de precisão \boldsymbol{R}$, com dimensão $n \times n$, é definida como a seguir:

$$
\boldsymbol{R}_{i j}=\tau\left\{\begin{array}{cc}
n_{i}, & \text { se } i=j, \\
-1, & \text { se } i \sim j, \\
0, & \text { em outro caso. }
\end{array}\right.
$$

Esta especificação é denominada como SBDR-Icar model.

Da parametrização ICAR, $\tau$ pode simbolizar dispersão a mais e dependência espacial ao mesmo tempo, veja Leroux et al. (2000). Por esta razão, também considera-se a proposta do Leroux et al. (2000) para o efeito aleatório $\boldsymbol{z}$. Esta proposta sugere uma modificação na matriz de precisão de $\boldsymbol{z}$ como a seguir:

$$
\boldsymbol{R}^{\prime}=\tau^{\prime}((1-\lambda) \boldsymbol{I}+\lambda \boldsymbol{R})
$$

onde $\tau^{\prime}$ é o parâmetro de precisão da matriz $\boldsymbol{R}^{\prime}, \boldsymbol{I}$ é a matriz de identidade, $\boldsymbol{R}$ é definido como na equação (3.6) com uma precisão de um $(\tau=1)$ e $\lambda$ é o parâmetro de dependência espacial com suporte no intervalo [0,1]. Quando $\lambda=0, \boldsymbol{z}$ têm componentes independentes o qual fornece o modelo denominado como SBDR-Ind (sem relações espaciais) e preserva dependência espacial quando $\lambda=1$ então, para este caso, o modelo SBDR-Icar é obtido. Em outros casos, quando $0<\lambda<1$, o modelo é denominado como SBDR-Ler, sendo $\lambda$ o grau de dependência espacial. Quando não são considerados os efeitos aleatórios, denomina-se o modelo de regressão limitado (BR), por exemplo o modelo de regressão Beta, o qual foi aplicado na Seção 3.2. 


\subsection{Inferência Bayesiana}

A inferência Bayesiana é utilizada com o intuito de ajustar o modelo definido nas equações (3.5), (3.6) e (3.7). Para este caso, o interesse é obter a distribuição a posteriori de $\mathrm{p}(\boldsymbol{z}, \boldsymbol{\beta}, \phi, \tau, \lambda \mid \boldsymbol{y})$.

Considerando as definições nas equações (3.6) e (3.7) a função de verossimilhança aumentada toma a seguinte forma:

$$
\begin{aligned}
& \mathrm{L}(\boldsymbol{\beta}, \phi, \tau, \lambda \mid \boldsymbol{y}, \boldsymbol{z})=\prod_{i=1}^{n} \mathrm{p}\left(y_{i} \mid \theta_{i}, \phi\right) \mathrm{p}(\boldsymbol{z} \mid \tau, \lambda) \\
& \propto \prod_{i=1}^{n} \mathrm{p}\left(y_{i} \mid \theta_{i}, \phi\right) \times \tau^{\frac{r\left(\boldsymbol{R}^{\prime}\right)}{2}} \exp \left\{-\frac{\tau}{2} \boldsymbol{z}^{\top} \boldsymbol{R}^{\prime} \boldsymbol{z}\right\}
\end{aligned}
$$

onde $\boldsymbol{\beta}, \phi, \tau$ e $\lambda$ são parâmetros os quais serão estimados no modelo, com $\theta_{i}=g^{-1}\left(\boldsymbol{x}_{\boldsymbol{i}}^{\boldsymbol{T}} \boldsymbol{\beta}+z_{i}\right)$.

Distribuições normais independentes são consideradas como distribuições a priori para cada um dos parâmetros $\boldsymbol{\beta}$ e $\operatorname{logit} \lambda, \log \left(\frac{\lambda}{1-\lambda}\right)$, as quais têm média zero e precisão pequena. Isto é, $\beta_{j} \sim \mathcal{N}\left(0, \tau_{\beta}\right), j=0,1, \ldots, p$ e logit $\lambda \sim \mathcal{N}\left(0, \tau_{\lambda}\right)$. Para o parâmetro $\phi$ foi adotado a distribuição a priori $\operatorname{Gamma}(a, b) \operatorname{com} a=1$ e $b=0.1$ a qual fornece uma média de 10 e variância de 100 Bonat et al. (2014). Finalmente, para o parâmetro $\tau$ foi considerado uma distribuição a priori com informação mínima assumindo a distribuição $\operatorname{Gamma}\left(a^{\prime}, b^{\prime}\right)$, onde $a^{\prime}=1$ e $b^{\prime}=0.0005$, a qual alcanza uma média de 2000 e uma variância maior (Blangiardo e Cameletti (2015) pg. 182).

Então, a distribuição a posteriori toma a seguinte forma:

$$
\begin{aligned}
\mathrm{p}(\boldsymbol{z}, \boldsymbol{\beta}, \phi, \tau, \lambda \mid \boldsymbol{y}) & =\mathrm{p}(\boldsymbol{y} \mid \boldsymbol{z}, \boldsymbol{\beta}, \phi) \mathrm{p}(\boldsymbol{z} \mid \tau, \lambda) \mathrm{p}(\boldsymbol{\beta}) \mathrm{p}(\phi) \mathrm{p}(\tau) \mathrm{p}(\lambda) \\
& =\mathrm{L}(\boldsymbol{\beta}, \phi, \tau, \lambda \mid \boldsymbol{y}, \boldsymbol{z}) \mathrm{p}(\boldsymbol{\beta}) \mathrm{p}(\phi) \mathrm{p}(\tau) \mathrm{p}(\lambda),
\end{aligned}
$$

isto é, a distribuição a posteriori é proporcional à função de verossimilhança, $\mathrm{p}(\boldsymbol{y} \mid \cdot) \mathrm{p}(\boldsymbol{z} \mid$.), multiplicada pela distribuição a priori dos parâmetros de interese. Neste caso, prioris independentes foram assumidas $\mathrm{p}(\boldsymbol{\beta}) \mathrm{p}(\phi) \mathrm{p}(\tau) \mathrm{p}(\lambda)$.

A distribuição a posteriori pode ser aproximada a través dos algoritmos de Cadeias de Markov Monte Carlo (MCMC); no entanto, foi adotado a aproximação de inferência Bayesiana para modelos latentes gaussianos utilizando o programa INLA. Informação geral sob o procedimento INLA pode ser achado no apêndice C.

Neste caso, para a aproximação INLA o modelo proposto toma o campo latente como $\boldsymbol{\zeta}=$ $(\boldsymbol{\theta}, \boldsymbol{\beta}, \boldsymbol{z})$ e os hiperparâmetros como $\boldsymbol{\gamma}=(\phi, \boldsymbol{\beta}, \tau, \lambda)$. Então, INLA retorna a distribuição marginal para o campo latente e a distribuição marginal para os hiperparâmetros.

\subsubsection{Critérios de comparação de modelos}

Como o modelo proposto é um modelo misto, vários critérios de comparação de modelos podem ser adotados.

Uma das medidas para escolher o melhor modelo é o critério de informação do desvio (DIC) o 
qual foi sugerido por Spiegelhalter et al. (2002), e é definido como a seguir:

$$
D I C=\hat{D}+p_{D}
$$

onde $\hat{D}$ é a média a posteriori do desvio e $p_{D}$ é o número efetivo de parâmetros para o modelo. Segundo Rue et al. (2009) seçaõ 6.4, o desvio é definico como

$$
D(\boldsymbol{\zeta}, \varrho)=-2 \sum_{i \in D} \log \mathrm{p}\left(y_{i} \mid \zeta_{i}, \boldsymbol{\varrho}\right)+\text { constant }
$$

onde $\varrho=(\phi, \boldsymbol{\beta}, \tau, \lambda)$ e o número efetivo de parâmetros é aproximado por

$$
p_{D} \approx n-\operatorname{Trace}\left\{\boldsymbol{Q} \times \boldsymbol{Q}^{*-1}\right\} .
$$

sendo $\boldsymbol{Q}$ a matriz de precisão a priori do campo latente $\boldsymbol{\zeta}$ e $\boldsymbol{Q}^{*-1}$ é a matriz de covariância a posteriori.

DIC é um dos critérios mais comuns, mas não existe consenso sob o uso dele. Portanto, outros critérios foram também utilizados tais como o critério de informação amplamente aplicado (WAIC) e o critério do erro absoluto médio (MAE).

O WAIC é sugerido por Watanabe (2009) e é definido como uma aproximação de ajuste da densidade preditiva logarítmica esperada "out-of-sample". Primeiro, é preciso calcular a densidade preditiva a posteriori, logo, adicionar uma correção para o número de parâmetros efetivos. Segundo Gelman et al. (2014), no contexto do presente trabalho, WAIC é definido como duas vezes a expressão (3.11)

$$
\text { elppd } \widehat{\mathrm{d}}_{\mathrm{WIC}}=\operatorname{lppd}-\mathrm{p}_{\mathrm{WAIC}}
$$

onde o lppd é o logaritmo da densidade preditiva pontual para $y_{i}$, a qual é induzida pela distribuição a posteriori $p(\varrho \mid \boldsymbol{y})$, determinado pela seguinte expressão

$$
\sum_{i=1}^{n} \log \int\left(\int \mathrm{p}\left(y_{i} \mid \zeta_{i}, \varrho\right) \mathrm{p}\left(\zeta_{i} \mid \boldsymbol{\varrho}, \boldsymbol{y}_{-i}\right) d \zeta_{i}\right) d \varrho
$$

e

$$
\mathrm{p}_{\mathrm{WAIC}}=\sum_{i=1}^{n} \operatorname{Var}\left(\log \int\left(\int \mathrm{p}\left(y_{i} \mid \zeta_{i}, \boldsymbol{\varrho}\right) \mathrm{p}\left(\zeta_{i} \mid \varrho, \boldsymbol{y}_{-i}\right) d \zeta_{i}\right) d \varrho\right) .
$$

Como foi sugerido por Blangiardo e Cameletti (2015), índices de resumo podem ser calculados utilizando a distribuição preditiva a posteriori $p\left(y_{i}^{\star} \mid \boldsymbol{y}\right)=\int \mathrm{p}\left(y_{i}^{\star} \mid \zeta_{i}\right) \mathrm{p}\left(\zeta_{i} \mid \boldsymbol{y}\right) d \zeta_{i}$, em outras palavras, a verossimilhança da observação replicada $y_{i}^{\star}$ dado o conjunto de dados $\boldsymbol{y}$. Logo, com o propósito de avaliação do modelo, define-se o critério denominado MAE, o qual é a diferença média absoluta entre o valor observado $y_{i}$ e o respectivo valor predito $y_{i}^{\star}$ para $i=1, \ldots, n$, a través da seguinte equação:

$$
\mathrm{MAE}=\frac{1}{n} \sum_{i=1}^{n}\left|y_{i}^{\star}-y_{i}\right|
$$

Assim, MAE mensura o desvio entre o valor observado $y_{i}$ e o valor predito $y_{i}^{\star}$ o qual fornece uma medida de bondade de ajuste. 


\subsection{Simulações}

A presente seção analisa a performance na recuperação dos parâmetros para o modelo proposto SBDR através de um estudo de simulação. Adicionalmente, avalia-se os critérios de comparação dos modelos mediante outros estudos de simulação específicos.

\subsubsection{Estudo de recuperação}

Neste estudo, nove cenários com $R e=100$ réplicas em cada um foram simulados para o modelo SBDR-Ler, pois este modelo é o mais geral. Os modelos em cada cenário têm distintos valores para o parâmetro $\tau:$ 0.5, 1,2 em cada uma das três distribuições limitadas: Beta, Simplex e Kumaraswamy.

O propósito da comparação dos modelos utilizando as três distribuições limitadas é achar características no desempenho de cada uma das distribuições. Ao mesmo tempo distintas quantidades no parâmetro $\tau$ podem identificar características de tal parâmetro, o qual simboliza a precisão das relações espaciais entre regiões.

A quantidade da dependência espacial para cada um dos cenários foi fixada como sendo $\lambda=0.9$. Com o intuito de preservar a comparação com os dados da aplicação, a configuração da vizinhança é idêntica à configuração da aplicação. Isto é, a simulação foi desenvolvida para $n=195$ regiões. Cada uma tem uma localização no espaço com valores de latitude e longitude, tais quantidades foram utilizadas para a elaboração da matriz $\boldsymbol{R}$ a qual contém as relações de dependência entre regiões. O vetor aleatório $\boldsymbol{z}$ foi simulado da distribuição normal multivariada com média zero e matriz de covariância $\boldsymbol{R}^{\prime-1}$, onde $\boldsymbol{R}^{\prime}$ é a matriz de precisão definida na equação (3.7). Os parâmetros da população $\boldsymbol{\beta}$ foram fixados com valores similares aos estimados obtidos da aplicação, $\boldsymbol{\beta}=(-3,2,0.7,1.5)$. As quantidades das covariáveis $\boldsymbol{x}_{i}, i=1, \ldots, n$ foram simuladas utilizando três distribuições independentes, as quais são respectivamente: $\operatorname{Beta}\left(\mu=0.2, \phi_{1}=15\right), \mathcal{N}(0,1)$ e Bernoulli(0.7). A intenção de tal seleção é ter uma ampla variedade de covariáveis considerando proporções, variáveis contínuas e categóricas. O parâmetro $\phi$ para cada distribuição foi selecionado como sendo semelhante à estimativa pontual da aplicação. Isto é: $\phi_{1}=80, \phi_{2}=19$ e $\phi_{3}=12$. Para cada cenário, o qual é um modelo gerado considerando uma distribuição limitada e uma quantidade de $\tau$, os modelos SBDR-Ler, SBDR-Icar e BR foram ajustados segundo a metodologia especificada na Seção 3.5. Com a intenção de avaliar a recuperação dos parâmetros, o viés absoluto (VA) foi definido como $\mathrm{VA}_{m}=\frac{\sum_{r=1}^{R e}\left|\hat{m}_{r}-m\right|}{R e}$, onde $m$ identifica o parâmetro em avaliação. Ademais, o desvio padrão (DP) e o número de vezes em que o parâmetro esta contido no intervalo de credibilidade de $95 \%$ (IC) foram reportados.

A Tabela 4.1 apresenta os resultados obtidos das respostas simuladas só para o modelo SBDR-Ler $\operatorname{com} \tau=1$ e três distribuições limitadas. É possível observar que a estimação pontual dos parâmetros de regressão são bastante precisos, $\mathrm{AB}$ mostra que os modelos com o viés absoluto do componente espacial mais pequeno que a versão sem o efeito aleatório espacial. Ademais, é possível perceber que o parâmetro $\phi$ acomoda melhor o verdadeiro esquema gerador e contém viés para o modelo ICAR e inclusive com maior viés para o modelo sem efeitos aleatórios. Para o modelo verdadeiro, o 
IC empírico é adequado, para o caso das respostas Beta e Kumaraswamy o IC apresenta resultados menos satisfatórios para $\phi$ e $\tau$ os quais são complicados de dividir pois ambos controlam toda a precisão do modelo. Adicionalmente, no caso da distribuição Beta o parâmetro $\phi$ tem uma escala maior em comparação ao caso das distribuições Kumaraswamy e Simplex. Ademais, a distribuição Kumaraswamy não consegue ter uma precisão pequena em comparação com a distribuição Simplex particularmente para $\theta<0.5$, veja a Figura 3.4 e Figuras no apêndice B. Os cenários considerando $\tau=0.5, \tau=2$ no estudo de simulação são incluídos no apêndice $\mathrm{D}$, os quais apresentam resultados similares.

\subsubsection{Estudo dos critérios de comparação dos modelos}

Em relação ao critério de seleção dos modelos, dois estudos foram desenvolvidos para conhecer a capacidade dos critérios de seleção do verdadeiro modelo. Para ambos casos, 100 conjuntos de dados foram simulados do modelo SBDR-Ler com cada uma das três distribuiçôes limitadas e $\tau=1$.

No primeiro estudo, no interior da mesma distribuição de resposta, a capacidade dos critérios de comparação dos modelos para selecionar o verdadeiro modelo gerador foi comparado, analisando os modelos SBDR-Ler, SBD-Icar e o modelo sem o efeito aleatório $z$ (BR). A Tabela 4.2 apresenta o número de vezes (para 100 conjuntos de dados) em que o critério seleciona o melhor modelo ajustado. É possível observar que os critérios, em todos os casos, atingem uma seleção certa, fazendo o modelo SBDR-Ler como um dos preferíveis em comparação com os modelos SBDR-Icar e BR.

O segundo estudo compara entre distintas respostas limitadas, isto é diferentes verossimilhanças, a capacidade dos critérios de comparação de modelos para determinar o modelo com a verdadeira distribuição de resposta.

A Tabela 3.5 confirma que os critérios de comparação dos modelos podem não ser conclusivos para selecionar o verdadeiro modelo, considerando as distribuições limitadas estudadas. Com a intenção de mostrar esta afirmação, foi fixado $\tau=1$ e $\lambda=0.9$ e dois experimentos foram desenvolvidos: Simulação A fixa $\phi_{3}=12, \phi_{2}=19$ e $\phi_{1}=80$ para as distribuições Kumaraswamy, Simplex e Beta, respectivamente. A simulação B define $\phi_{3}=1$ para a distribuição Kumaraswamy, $\phi_{2}=5$ para a distribuição Simplex e $\phi_{1}=10$ para a distribuição Beta.

Podemos apreciar na Tabela 3.5 que para quantidades maiores de $\phi$ a distribuição Simplex ajusta muito bom ao conjunto de dados e domina a seleção para todos os critérios inclusive quando não é a verdadeira distribuição. Porém, para pequenas quantidades de $\phi$ os critérios selecionam melhor o verdadeiro modelo. O critério MAE apresenta um desempenho precário quando a distribuição Beta é o verdadeiro modelo gerador. O qual é uma evidencia que para uma escala pequena de $\phi$ as distribuições têm comportamento diferenciado e não é possível ajustar bem os dados gerados. Este resultado pode ser explicado pois as distribuições limitadas são bastante flexíveis para distintas combinações dos parâmetros.

\subsection{Análise com dados reais}

Na presente seção, o conjunto de dados da prova de Compreensão Leitora (CL), analisado na Seção 3.2, é revisitado e uma análise de regressão comparativa é desenvolvida, na qual considerase ou não o componente espacial $\boldsymbol{z}$ para o conjunto de dados avaliado. Assim, os modelos SBDR (SDBR-Ler, SDBR-Icar) e o modelo sem o componente espacial $\boldsymbol{z}$ (BR) foram ajustados usando as 


\begin{tabular}{|c|c|c|c|c|c|c|c|c|}
\hline & Parâmetro & $\beta_{0}$ & $\beta_{1}$ & $\beta_{2}$ & $\beta_{3}$ & $\phi$ & $\tau$ & $\lambda$ \\
\hline \multicolumn{9}{|c|}{ Distribuição Beta } \\
\hline \multirow{5}{*}{ SBDR-Ler } & Original & -3 & 2 & 0.7 & 1.5 & 80 & 1 & 0.9 \\
\hline & Estimativa & -2.973 & 2.001 & 0.692 & 1.479 & 60.995 & 1.398 & 0.864 \\
\hline & DP & 0.309 & 0.433 & 0.041 & 0.101 & 16.655 & 0.321 & 0.095 \\
\hline & VA & 0.218 & 0.351 & 0.034 & 0.083 & 19.72 & 0.417 & 0.062 \\
\hline & Cobertura do $95 \%$ IC & 94 & 95 & 95 & 91 & 79 & 83 & 96 \\
\hline \multirow[t]{4}{*}{ SBDR-Icar } & Estimativa & -2.96 & 1.994 & 0.689 & 1.471 & 55.578 & 1.466 & \\
\hline & DP & 0.125 & 0.424 & 0.04 & 0.101 & 14.348 & 0.377 & \\
\hline & VA & 0.219 & 0.353 & 0.035 & 0.084 & 24.772 & 0.484 & \\
\hline & Cobertura do $95 \%$ IC & 66 & 95 & 95 & 91 & 62 & 80 & \\
\hline \multirow[t]{4}{*}{$\mathrm{BR}$} & Estimativa & -2.647 & 1.808 & 0.62 & 1.275 & 13.537 & & \\
\hline & DP & 0.152 & 0.471 & 0.045 & 0.121 & 1.348 & & \\
\hline & VA & 0.37 & 0.5 & 0.083 & 0.225 & 66.463 & & \\
\hline & Cobertura do $95 \%$ IC & 47 & 87 & 54 & 54 & 0 & & \\
\hline \multicolumn{9}{|c|}{ Distribuição Kumaraswamy } \\
\hline & True & -3 & 2 & 0.7 & 1.5 & 12 & 1 & 0.9 \\
\hline \multirow[t]{4}{*}{ SBDR-Ler } & Estimativa & -3.004 & 2.035 & 0.7 & 1.507 & 8.888 & 1.299 & 0.862 \\
\hline & DP & 0.315 & 0.405 & 0.037 & 0.09 & 1.461 & 0.231 & 0.093 \\
\hline & VA & 0.215 & 0.317 & 0.029 & 0.069 & 3.134 & 0.306 & 0.064 \\
\hline & Cobertura do $95 \%$ IC & 89 & 97 & 95 & 95 & 52 & 78 & 97 \\
\hline \multirow[t]{4}{*}{ SBDR-Icar } & Estimativa & -3.005 & 2.036 & 0.7 & 1.509 & 8.51 & 1.27 & \\
\hline & DP & 0.11 & 0.398 & 0.036 & 0.09 & 1.379 & 0.241 & \\
\hline & VA & 0.215 & 0.321 & 0.029 & 0.068 & 3.51 & 0.28 & \\
\hline & Cobertura do $95 \%$ IC & 59 & 97 & 94 & 95 & 32 & 83 & \\
\hline \multirow[t]{4}{*}{$\mathrm{BR}$} & Estimativa & -2.97 & 2.011 & 0.697 & 1.497 & 3.107 & & \\
\hline & DP & 0.18 & 0.521 & 0.048 & 0.15 & 0.162 & & \\
\hline & VA & 0.248 & 0.54 & 0.044 & 0.106 & 8.893 & & \\
\hline & Cobertura do $95 \%$ IC & 72 & 86 & 92 & 99 & 0 & & \\
\hline \multicolumn{9}{|c|}{ Distribuição Simplex } \\
\hline \multirow{5}{*}{ SBDR-Ler } & Original & -3 & 2 & 0.7 & 1.5 & 19 & 1 & 0.9 \\
\hline & Estimativa & -3.016 & 2.032 & 0.696 & 1.503 & 12.379 & 1.125 & 0.845 \\
\hline & DP & 0.259 & 0.345 & 0.034 & 0.077 & 7.338 & 0.212 & 0.106 \\
\hline & VA & 0.205 & 0.271 & 0.026 & 0.06 & 6.639 & 0.168 & 0.081 \\
\hline & Cobertura do $95 \%$ IC & 89 & 96 & 94 & 93 & 93 & 93 & 94 \\
\hline \multirow[t]{4}{*}{ SBDR-Icar } & Estimativa & -3.016 & 2.033 & 0.696 & 1.504 & 10.606 & 1.054 & \\
\hline & DP & 0.087 & 0.357 & 0.032 & 0.07 & 8.081 & 0.137 & \\
\hline & VA & 0.205 & 0.27 & 0.027 & 0.06 & 8.407 & 0.132 & \\
\hline & Cobertura do $95 \%$ IC & 51 & 95 & 93 & 92 & 83 & 92 & \\
\hline \multirow[t]{4}{*}{$\mathrm{BR}$} & Estimativa & -2.836 & 1.895 & 0.651 & 1.439 & 0.248 & & \\
\hline & DP & 0.131 & 0.536 & 0.043 & 0.097 & 0.025 & & \\
\hline & VA & 0.262 & 0.515 & 0.068 & 0.12 & 18.752 & & \\
\hline & Cobertura do $95 \%$ IC & 53 & 92 & 66 & 77 & 0 & & \\
\hline
\end{tabular}

Tabela 3.3: Estudo de recuperação dos parâmetros para o modelo simulado SBDR-Ler, considerando três distribuições limitadas e precisão espacial $\tau=1$

\begin{tabular}{|c|c|c|c|}
\hline Critério & SBDR-Ler & SBDR-Icar & $\mathrm{BR}(\operatorname{sem}$ o $\boldsymbol{z})$ \\
\hline \multicolumn{4}{|c|}{ Distribuição Beta } \\
\hline DIC & 99 & 1 & 0 \\
\hline WAIC & 100 & 0 & 0 \\
\hline MAE & 100 & 0 & 0 \\
\hline \multicolumn{4}{|c|}{ Distribuição Kumaraswamy } \\
\hline DIC & 100 & 0 & 0 \\
\hline WAIC & 100 & 0 & 0 \\
\hline MAE & 100 & 0 & 0 \\
\hline \multicolumn{4}{|c|}{ Distribuição Simplex } \\
\hline DIC & 68 & 32 & 0 \\
\hline WAIC & 97 & 3 & 0 \\
\hline MAE & 100 & 0 & 0 \\
\hline
\end{tabular}

Tabela 3.4: Avaliação dos critérios de comparação de modelos DIC, WAIC e MAE para selecionar o verdadeiro modelo nos dados simulados do modelo SBDR-Ler considerando respostas das diferentes distribuições limitadas com precisão espacial $\tau=1$. As quantidades são o número de vezes em 100 conjuntos de dados. 


\begin{tabular}{|c|c|c|c|c|c|c|}
\hline \multirow[b]{2}{*}{ Critério } & \multicolumn{3}{|c|}{ Simulação A } & \multicolumn{3}{|c|}{ Simulação B } \\
\hline & Beta & Simplex & Kumaraswamy & Beta & Simplex & Kumaraswamy \\
\hline \multicolumn{4}{|c|}{ SBDR-Ler Beta $\phi=80$} & \multicolumn{3}{|c|}{ SBDR-Ler Beta $\phi=10$} \\
\hline DIC & 4 & 92 & 4 & 69 & 1 & 30 \\
\hline WAIC & 6 & 92 & 2 & 79 & 1 & 20 \\
\hline MAE & 4 & 92 & 4 & 19 & 1 & 80 \\
\hline \multicolumn{4}{|c|}{ SBDR-Ler Simplex $\phi=19$} & \multicolumn{3}{|c|}{ SBDR-Ler Simplex $\phi=5$} \\
\hline DIC & 0 & 93 & 7 & 0 & 89 & 11 \\
\hline WAIC & 0 & 90 & 10 & 0 & 85 & 15 \\
\hline MAE & 0 & 80 & 20 & 0 & 68 & 32 \\
\hline \multicolumn{4}{|c|}{ SBDR-Ler Kumaraswamy $\phi=12$} & \multicolumn{3}{|c|}{ SBDR-Ler Kumaraswamy $\phi=1$} \\
\hline DIC & 0 & 100 & 0 & 27 & 0 & 73 \\
\hline WAIC & 0 & 100 & 0 & 35 & 0 & 65 \\
\hline MAE & 0 & 100 & 0 & 10 & 0 & 90 \\
\hline
\end{tabular}

Tabela 3.5: Número de vezes selecionado como o melhor modelo sob os critérios DIC, WAIC e MAE, para modelo simulado SBDR-Ler com uma precisão espacial de $\tau=1$

três distribuições limitadas estudadas no presente capítulo.

O propósito na análise dos dados reais é explicar a proporção de estudantes com nível satisfatório na prova de CL $(y)$ para cada uma das 195 províncias peruanas (divisão política de segundo ordem) com três variáveis explanatórias como covariáveis: os índices de Saúde, Saneamento e Electrificação os quais são simbolizados como $x_{1}, x_{2}$ e $x_{3}$, respectivamente.

Aspectos adicionais em relação à prova de CL são descritos no documento de fundamentação MINEDU (2016). Os índices de Saúde, Saneamento e Electrificação são peças do índice de densidade do estado os quais são detalhados na seção 3.2 e especificações adicionais podem ser encontradas no documento do programa das nações unidas para o desenvolvimento PNUD (2013).

Como foi descrito na seção 3.2 e na Figura E.2, observa-se que os resíduos, os quais não são explicados no modelo de regressão Beta habitual para o nível satisfatório na prova de CL, têm uma configuração espacial a qual é considerada no modelo proposto SBDR como uma variável aleatória $\boldsymbol{z}$, a qual sintetiza as relações em províncias contíguas (relações espaciais).

Especificamente, para esta aplicação define-se o modelo SBDR como a seguir:

$$
\begin{aligned}
& y_{i} \mid z_{i}, \phi \stackrel{i n d .}{\sim} \pi\left(\theta_{i}, \phi\right) \\
& g\left(\theta_{i}\right)=\beta_{0}+\beta_{1} x_{1 i}+\beta_{2} x_{2 i}+\beta_{3} x_{3 i}+z_{i} \\
& z_{i} \mid \boldsymbol{z}_{-i}, \tau^{\prime}, \lambda \sim \mathcal{N}\left(\frac{\lambda}{1-\lambda+\lambda n_{i}} \sum_{i \sim j} z_{j}, \frac{1}{\left(1-\lambda+\lambda n_{i}\right) \tau^{\prime}}\right) \\
& i=1, \ldots, 195
\end{aligned}
$$

onde $\boldsymbol{z}=\left(z_{1}, \ldots, z_{195}\right)^{\prime}$ é o vetor aleatório o qual preserva o efeito espacial e segue a definição Leroux Leroux et al. (2000) denominado como o modelo SBDR-Ler. Quando o $\lambda=1$ o modelo é chamado SBDR-Icar e quando $\lambda=0$ define-se o modelo SBDR-Ind. A distribuição $\pi\left(\theta_{i}, \phi\right)$ pode considerar as três distribuições limitadas: Beta, Simplex e Kumaraswamy onde $\theta_{i}$ é o parâmetro de localização 


\begin{tabular}{|c|c|c|c|}
\hline \multicolumn{4}{|c|}{ O modelo SBDR-Ler Beta } \\
\hline & Média & $\mathrm{DP}$ & $95 \%$ IC \\
\hline$\beta_{0}$ & -3.54 & 0.24 & $(-4.01,-3.07)$ \\
\hline$\beta_{1}$ & 1.62 & 0.35 & $(0.93,2.31)$ \\
\hline$\beta_{2}$ & 0.84 & 0.26 & $(0.34,1.35)$ \\
\hline$\beta_{3}$ & 1.67 & 0.30 & $(1.09,2.27)$ \\
\hline$\phi$ & 82.73 & 17.52 & $(53.36,121.89)$ \\
\hline$\tau$ & 2.86 & 0.59 & $(1.87,4.19)$ \\
\hline$\lambda$ & 0.90 & 0.06 & $(0.74,0.98)$ \\
\hline \multicolumn{4}{|c|}{$\overline{D I C}=-628$} \\
\hline \multicolumn{4}{|c|}{ WAIC $=-642$} \\
\hline \multicolumn{4}{|c|}{$\mathrm{MAE}=0.0209$} \\
\hline \multicolumn{4}{|c|}{ O modelo SBDR-Ler Simplex } \\
\hline & Média & DP & $95 \% \mathrm{IC}$ \\
\hline$\beta_{0}$ & -3.59 & 0.23 & $(-4.04,-3.14)$ \\
\hline$\beta_{1}$ & 1.74 & 0.38 & $(0.99,2.49)$ \\
\hline$\beta_{2}$ & 0.82 & 0.27 & $(0.29,1.35)$ \\
\hline$\beta_{3}$ & 1.68 & 0.30 & $(1.08,2.28)$ \\
\hline$\phi$ & 19.52 & 12.13 & $(5.16,50.85)$ \\
\hline$\tau$ & 1.40 & 0.18 & $(1.08,1.77)$ \\
\hline$\lambda$ & 0.81 & 0.09 & $(0.60,0.95)$ \\
\hline \multicolumn{4}{|c|}{$\mathrm{DIC}=-1022$} \\
\hline \multicolumn{4}{|c|}{ WAIC $=-1055$} \\
\hline \multicolumn{4}{|c|}{$\mathrm{MAE}=0.0028$} \\
\hline \multicolumn{4}{|c|}{ O O modelo SBDR-Ler Kumaraswamy } \\
\hline & Média & $\mathrm{DP}$ & $95 \%$ IC \\
\hline$\beta_{0}$ & -3.54 & 0.24 & $(-4.01,-3.06)$ \\
\hline$\beta_{1}$ & 1.66 & 0.35 & $(0.98,2.34)$ \\
\hline$\beta_{2}$ & 0.77 & 0.25 & $(0.28,1.27)$ \\
\hline$\beta_{3}$ & 1.72 & 0.30 & $(1.14,2.30)$ \\
\hline$\phi$ & 11.25 & 1.71 & $(8.24,14.95)$ \\
\hline$\tau$ & 2.09 & 0.35 & $(1.49,2.87)$ \\
\hline$\lambda$ & 0.88 & 0.07 & $(0.69,0.98)$ \\
\hline \multicolumn{4}{|c|}{$\mathrm{DIC}=-723$} \\
\hline \multicolumn{4}{|c|}{ WAIC $=-733$} \\
\hline \multicolumn{4}{|c|}{$\mathrm{MAE}=0.0133$} \\
\hline
\end{tabular}

Tabela 3.6: Média à posteriori (média), desvio padrão (DP) e o $95 \%$ do intervalo de credibilidade (IC) para os parâmetros estimados do conjunto de dados RC sob os modelos SBDR-Ler

ou posição e $\phi$ é parâmetro de precisão. Adicionalmente, $g(s)=\log (s /(1-s))$ é a função de ligação logit. A regressão não espacial pode ser ilustrada através da equação (3.13) mas sem o efeito espacial $\boldsymbol{z}$ e sua distribuição a priori denominada como BR model. O código utilizado para esta aplicação é incluído no apêndice E.

A Tabela E.1 apresenta só a estimação dos parâmetros para os possíveis modelos SBDR-Ler e os respectivos critérios de comparação dos modelos DIC, WAIC e MAE. Adicionalmente, a Tabela A1 no apêndice E contém informação equivalente para os modelos SDBR-Icar e o análogo BR sem o componente espacial $\boldsymbol{z}$. Sob os critérios de comparação de modelos (DIC, WAIC e MAE), o modelo SBDR-Ler Simplex foi escolhido sendo o melhor modelo que ajusta os dados de CL. Ademais, é possível observar que tal modelo apresenta o melhor resultado em relação aos modelos alternativos no interior da família Simplex, veja a Tabela D.1 do apêndice E a qual apresenta os critérios de ajuste para os modelos adversários. Além disso, o resíduo padrão e o PPD do modelo SBDR-Ler Simplex são apresentados na Figura 3.5 e para os modelos alternativos no apêndice. Tais figuras sugerem uma redução dos resíduos os quais não apresentam uma configuração espacial, veja Figura 3.6, tal como foi observado na análise preliminar na Seção 3.2. 

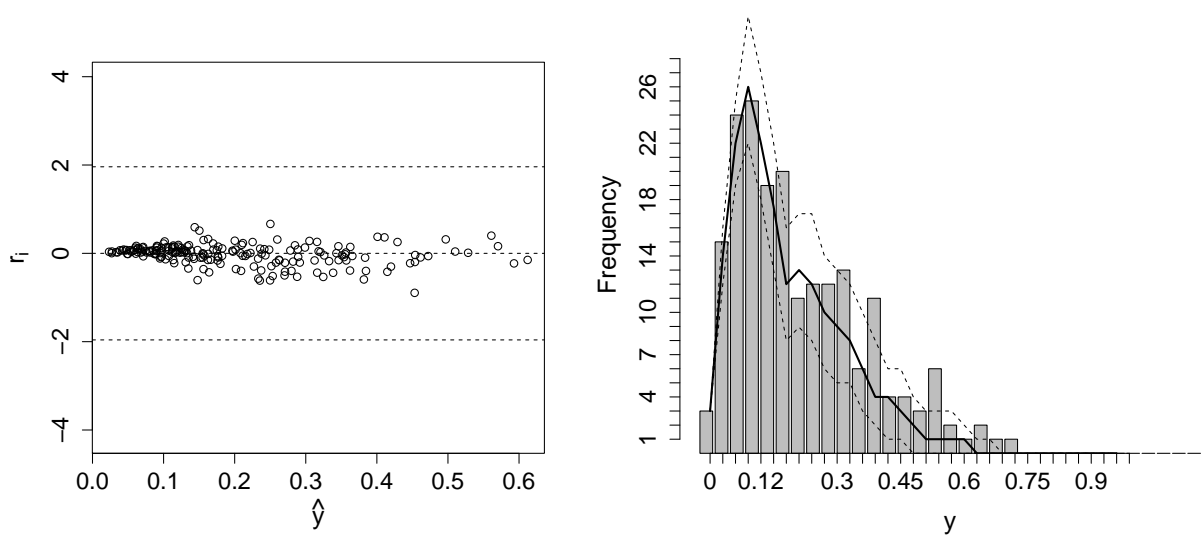

Figura 3.5: Ajuste do modelo SBDR-Ler Simplex para o conjunto de dados de CL. a) Resíduos padrão ( $r_{i}$ ) versus respostas ajustadas. b) Banda de confiança da distribuição preditiva a posteriori com o histograma da variável de resposta observada.

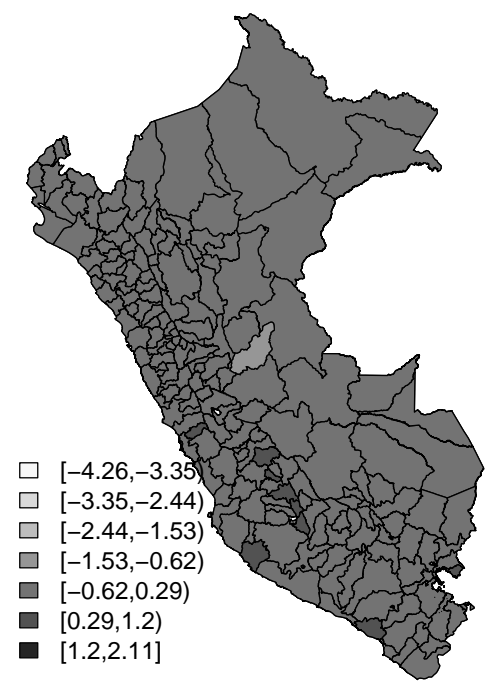

Figura 3.6: Mapa das 195 provincias peruanas para o conjunto de dados de CL com os resíduos padrão do modelo SBDR-Ler Simplex. 


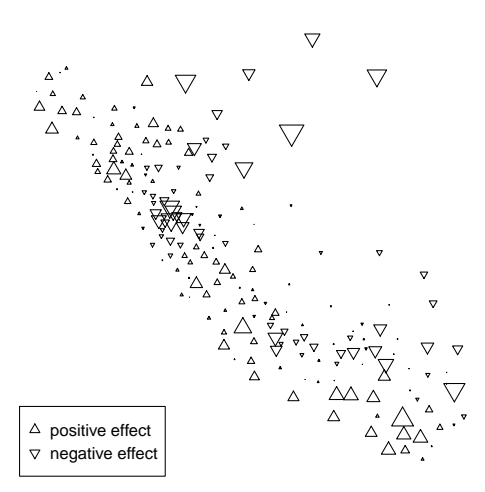

Figura 3.7: Média a posteriori dos efeitos espaciais para o conjunto de dados de CL.

Recentemente o confundimento espacial entre os efeitos fixos e efeitos aleatórios espaciais foram tratados na literatura (Hodges e Reich, 2010; Reich et al., 2006). Possíveis soluções e discusões em relação à necessidade de tal reparo surgiram, veja por exemplo (Hanks et al., 2015; Hughes e Haran, 2013; Prates et al., 2019; Thaden e Kneib, 2018). A versão sem o confundimento espacial do método Leroux, chamado SBDR-SPOCK, foi ajustada, pois o método SPOCK (Prates et al., 2019) pode ser ajustado usando INLA. A Tabela D.1 no apêndice E revela que o modelo SBDR-Ler Simplex ainda apresenta o menor DIC, WAIC e com MAE semelhante, o qual sugere que este modelo é preferível. Assim, retomamos as análises com o modelo SBDR-Ler Simplex.

Considerando o modelo escolhido, o $95 \%$ do intervalo de credibilidade sugere que o parâmetro $\beta_{0}$ é negativo. Utilizando a estimativa da média a posteriori é possível avaliar o inverso do logit $g^{-1}\left(\beta_{0}\right)=1 /(1+\exp (3.59))=0.03$ o qual pode ser interpretado de tal forma que se uma província a qual não fornece saúde, saneamento e eletrificação só obtém $3 \%$ dos estudantes com nível satisfatório em CL. Inclusive, o 95\% do intervalo de credibilidade para os $\beta_{1}, \beta_{2}$ e $\beta_{3}$ não contém a quantidade zero, o qual sugere a existência de um efeito positivo dos índices de saúde, saneamento e eletrificação em relação à proporção de estudantes com nível satisfatório na prova de CL para as províncias. O qual implica que quantidades maiores nos índices têm um efeito positivo na quantidade de estudantes com nível satisfatório em CL para cada uma das províncias.

Os resultados revelam a existência do efeito espacial $\lambda=0.81$, onde a precisão associada com este efeito é $\tau=1.4$, o qual origina uma variação entre as quantidades de $z_{i}$. As estimativas de $z_{i}$ são reproduzidas na Figura (3.7), onde o tamanho dos triângulos estão em função das quantidades de $\boldsymbol{z}$. Na imagen é possível identificar efeitos positivos $(\triangle)$ e negativos $(\nabla)$ através do sentido dos triângulos. Efeitos positivos estão concentrados no sul das províncias peruanas. O nordeste (o Amazonas) têm efeitos espaciais negativos. 


\subsection{Comentários finais}

No presente trabalho, o modelo de regressão espacial para distribuições limitadas (SBDR) é proposto, onde o efeito espacial segue a definição de Leroux, a qual retorna o grau de dependência espacial $\lambda \in[0,1]$ e contém como casos particulares as definições de SBDR-Icar, SBDR-Ind e a definição sem o efeito espacial.

A distribuição a posteriori não é simples para uma abordagem analítica, por tal razão utiliza-se o método INLA. Os resultados do estudo de simulação mostram que a proposta Bayesiana retorna estimativas com bom desempenho. Além disso, um conjunto de dados reais é analisado utilizando a proposta. Na definição do modelo SBDR-Ler, a quantidade $\lambda$, a qual representa o efeito espacial no intervalo $[0,1]$, fornece uma medição do grau das relações espaciais presentes no modelo.

A proposta pressupõe mesmas distâncias entre regiões, embora, distâncias diferentes podem ser definidas no efeito espacial. Além disso, estimações Bayesianas utilizando o BUGS (Goudie et al. (in press); Lunn et al. (2000); Spiegelhalter et al. (2014)), JAGS (Plummer (2012)), STAN (Carpenter et al. (2017)) ou outras ferramentas de análise Bayesiana podem ser desenvolvidas. Finalmente, trabalhos futuros podem considerar a inclusão de regressão espacial para respostas elevadas zero/um com distribuições limitadas. 


\section{Capítulo 4}

\section{Modelos do tempo de resposta limitado e a precisão da resposta para o estudo da compreensão leitora}

\subsection{Introdução}

$\mathrm{Na}$ atualidade, com o uso de computadores particulares (PCs) em Avaliações, como por exemplo o uso de PCs no programa para avaliação internacional de estudantes (PISA) (do acrónimo em inglês Programme for International Student Assessment), o tempo que utiliza um estudante em solucionar uma prova torna-se naturalmente disponível. Com a disponibilidade desta informação, várias propostas de modelos utilizaram os tempos de resposta (RTs) como informação adicional na estimação da habilidade dos examinados, veja, por exemplo, Fox et al. (2007); Im (2015); Klein Entink (2009); van der Linden (2007); Zhan et al. (2018).

Existem estudos em relação ao uso do tempo de resposta (RT) em avaliações, onde a variável RT é definida como o período do tempo em que uma pessoa é capaz de ler e responder uma pergunta ou item de uma prova. Estes estudos estão enfocados na investigação de atividades mentais e foram desenvolvidos inclusive antes do estabelecimento formal da Psicologia, uma revisão de tais estudos é apresentada no trabalho do Schnipke e Scrams, veja Schnipke e Scrams (2002). Inicialmente, se pensou que o RT e a precisão mediam um constructo semelhante, porém investigações adicionais não apoiaram esta afirmação. Assim, a rapidez foi definida como um traço de personalidade diferente da precisão no trabalho de Kennedy (1930). Com base nesta definição da rapidez, isto é o RT para cada item de uma prova, e com a disponibilidade dos dados de RT, vários modelos para a rapidez foram sugeridos na literatura.

Uma classificação para estes modelos do RT, sugerida por Fox et al. (2007), é dada de três formas: A primeira ocupa os modelos de RT no contexto da TRI, a segunda modela o RT independentemente e a terceira modela o RT e a precisão de forma hierárquica considerando uma relação de permuta (tradeoff) entre a rapidez (medida do tempo de resposta) e a precisão (medida da certeza na resposta). Esta ultima forma torna explícita a relação empírica entre a rapidez e a precisão. 
O RT é uma variável com suporte no intervalo $[0, \infty>$. Assim, uma distribuição clássica assumida para modelar o RT é a distribuição Lognormal. Existem vários trabalhos, por exemplo, o trabalho de Schnipke e Scrams (1999), que mostra um bom desempenho da distribuição Lognormal. No entanto, pensando de maneira realista o tempo não é infinito. Ele tem um limite superior, pois a aplicação da prova é efetuada em um tempo definido. Portanto, o presente trabalho pesquisa o uso da distribuição limitada, veja o capítulo 3 seção 3.3, para modelar a proporção de tempo utilizado no item. Especificamente, considera-se as distribuições Beta Ferrari e Cribari-Neto (2004); Johnson et al. (1995) e Simplex Barndorff-Nielsen e JØrgensen (1991); JØrgensen (1997) com o intuito de modelar a proporção do tempo de resposta (RT) e apresenta-se também uma avaliação do desempenho do modelo proposto.

Adicionalmente, o modelo sugerido no presente capítulo é implementado na abordagem hierárquica proposta pelo autor van der Linden (2007). A abordagem hierárquica sugere o modelamento das respostas (precisão) e os tempos de resposta (rapidez) de forma conjunta. O modelo da precisão ou exatidão é chamado, no presente capítulo, como o componente IRT e o modelo da rapidez é denominado como a parte RT. Ambos os traços latentes modelados (precisão e rapidez) são definidos em dois níveis: o primeiro, para um nível individual, modela a relação ("tradeoff") entre a precisão e a rapidez, e o segundo esta em relação ao nível populacional, isto é para o grupo completo dos examinados.

A organização do presente capítulo é da seguinte forma: Na seção ??, apresenta-se a distribuição limitada, a qual é utilizada para modelar os RTs, na seção 4.3 expõe-se o modelo para o tempo de resposta limitado, o qual é utilizado no modelo hierárquico na seção ??. A estimação Bayesiana do modelo proposto é analisada na seção 4.5. Dois estudos de simulação para comparar o desempenho do modelo sugerido são apresentados na seção 4.6. Apresenta-se também na seção 4.7 uma aplicação do modelo com dados reais e comentários finais são abordados na seção 4.8 .

\subsection{A distribuição limitada}

Nesta seção introduzimos a definição da distribuição limitada a qual abrange três distribuições com suporte no intervalo $(0,1)$, veja 3.3 .

Definição 1. Considere uma variável aleatória $Y$, ela segue uma distribuição limitada, com notação $Y \sim \pi(\theta, \phi)$, se o suporte da variável aleatória é o intervalo $(0,1), \theta$ é o parâmetro de locação o posição e $\phi$ é o parâmetro de precisão.

Onde o parâmetro de posição $\theta$ pode ser modelado de forma direita, isto é $\theta=E(Y)$ ou $\theta=M(Y)$, onde $E$ define a esperança matemática e $M$ simboliza a mediana. Especificamente, consideramos a distribuição limitada como uma generalização de três distribuições univariadas: a distribuição Beta, a distribuição Simplex e a distribuição Kumaraswamy, sendo o parâmetro de posição $\mu=E(Y)$ no caso das distribuições Beta e Simplex e $\kappa$ em relação à mediana da distribuição Kumaraswamy. Ademais, $\phi$ é definido como o inverso da dispersão. A seguir especificamos a função de densidade de probabilidade das distribuições consideradas como membro da distribuição limitada. 
A distribuição Beta é membro da classe I ou II do sistema Pearson, veja Johnson et al. (1995), a forma particular com suporte no intervalo $(0,1)$, denotada como $B\left(\mu, \phi_{1}\right)$, com $\mu \in(0,1)$ sendo o parâmetro de posição e $\phi_{1}$ o parâmetro de precisão, tem a seguinte função de densidade de probabilidade:

$$
p\left(y \mid \mu, \phi_{1}\right)=\frac{\Gamma\left(\phi_{1}\right)}{\Gamma\left(\mu \phi_{1}\right) \Gamma\left[(1-\mu) \phi_{1}\right]} y^{\mu \phi_{1}-1}(1-y)^{(1-\mu) \phi_{1}-1} I_{(0,1)}(y) .
$$

com média e variância definidas da seguiente forma:

$$
\begin{aligned}
& E\left[Y \mid \mu, \phi_{1}\right]=\mu \mathrm{e} \\
& \operatorname{Var}\left[Y \mid \mu, \phi_{1}\right]=\frac{\mu(1-\mu)}{1+\phi_{1}} .
\end{aligned}
$$

A distribuição Simplex foi sugerida pelos autores Barndorff-Nielsen e JØrgensen (1991); JØrgensen (1997) sendo um caso particular da família geral chamada modelos de dispersão. A distribuição univariada Simplex, $S\left(\mu, \phi_{2}\right)$, com parâmetro de posição $\mu \in(0,1)$ e parâmetro de precisão $\phi_{2}>0$, é definida através da seguinte função de densidade de probabilidade:

$$
p\left(y \mid \mu, \phi_{2}\right)=\frac{\sqrt{\phi_{2}}}{\sqrt{2 \pi\{y(1-y)\}^{3}}} \exp \left[-\frac{\phi_{2}}{2} d(y \mid \mu)\right] I_{(0,1)}(y),
$$

onde $d(y \mid \mu)=\frac{(y-\mu)^{2}}{y(1-y) \mu^{2}(1-\mu)^{2}}$ é denominado como o desvio unitário, o qual é positivo e tem o valor zero quando $y=\mu$. A média e a variancia são definidos como a seguir:

$$
\begin{aligned}
E\left(Y \mid \mu, \phi_{2}\right)= & \mu \quad \mathrm{e} \\
\operatorname{Var}\left(Y \mid \mu, \phi_{2}\right)= & \mu(1-\mu)-\sqrt{\frac{\phi_{2}}{2}} \\
& \exp \left\{\frac{\phi_{2}}{2 \mu^{2}(1-\mu)^{2}}\right\} \\
& \Gamma\left(\frac{1}{2}, \frac{\phi_{2}}{2 \mu^{2}(1-\mu)^{2}}\right),
\end{aligned}
$$

$\operatorname{com} \Gamma(a, b)=\int_{b}^{\infty} t^{a-1} e^{-t} d t$ sendo a função gamma incompleta.

A distribuição Kumaraswamy é um caso particular da densidade de probabilidade seno-potência, no intervalo $(0,1)$, sugerida pelo autor P. Kumaraswamy, veja Kumaraswamy (1976). A função de densidade de probabilidade para esta distribuição, $K\left(\kappa, \phi_{3}\right)$, com parâmetro de posição $\kappa \in(0,1)$ e parâmetro de precisão $\phi_{3}$, é definida da seguinte forma:

$$
\begin{aligned}
p\left(y \mid \kappa, \phi_{3}\right)= & -\frac{\log (1-q) \phi_{3}}{\log \left(1-e^{-\phi_{3}}\right) \log (\kappa)} y^{-\frac{\phi_{3}}{\log (\kappa)}-1} \\
& \times\left\{1-y^{-\frac{\phi_{3}}{\log (\kappa)}}\right\}^{\frac{\log (1-q)}{\log \left(1-e^{\left.-\phi_{3}\right)}\right.}-1},
\end{aligned}
$$

onde, $\kappa=G^{-1}(q)$ com $q$ assumida como fixa, $q=0.5$, a qual define o parâmetro de posição como sendo a mediana e $G^{-1}($.) denota o inverso da função de distribuição acumulada (fda) $\mathrm{Ku}$ - 
maraswamy. A média e a variância são respetivamente:

$$
\begin{aligned}
& E\left(Y \mid \kappa, \phi_{3}\right)=\frac{\log (1-q)}{\log \left(1-e^{-\phi_{3}}\right)} B\left(1-\frac{\log \kappa}{\phi_{3}}, \frac{\log (1-q)}{\log \left(1-e^{-\phi_{3}}\right)}\right) \\
& \mathrm{e} \\
& \operatorname{Var}\left(Y \mid \kappa, \phi_{3}\right)=\frac{\log (1-q)}{\log \left(1-e^{-\phi_{3}}\right)} B\left(1-\frac{2 \log \kappa}{\phi_{3}}, \frac{\log (1-q)}{\log \left(1-e^{-\phi_{3}}\right)}\right) \\
& -\left(E\left(Y \mid \kappa, \phi_{3}\right)\right)^{2},
\end{aligned}
$$

onde $B(\cdot, \cdot)$ denota a função beta.

Algumas formas da densidade limitada são ilustradas na Figura 4.1. Podemos enxergar diferençãs da distribuição beta (parte superior), simplex (no médio) e a kumaraswamy (parte inferior) para quantidades fixas da posição $\theta$ e precisão $\phi$. A precisão tem uma escala diferenciada, tal como foi sugerido na seção 3.3. A simplex concentra a massa da distribuição ao redor da posição $\theta$ para uma quantidade pequena da precisão. Ademais, uma precisão muito baixa da simplex consegue modelar a bi-modalidade, veja Quintero (2017). Em quanto a beta, a concentração da massa ao redor da média é alcançada com uma quantidade maior da precisão, veja B, com a vantagem que as estimações não resultem em questões de "overflow". A kumaraswamy tem como parâmetro de posição a mediana com a interpretação da metade e não a concentração da massa, uma característica a salientar em esta densidade é o fato de no ter um reflexo da massa para a posição em relação ao ponto 0.5 , veja o apêndice B.

\subsection{Modelos para o tempo de resposta limitado (BRT)}

A presente seção sugere possibilidades de modelos para analisar o tempo de resposta (RT) como uma variável limitada. Quando um examinado é avaliado com a intenção de conhecer a habilidade dele, em geral é submetido a uma prova com um período de tempo fixo. Então, o examinado pode dividir o tempo que tem para cada item em função da habilidade ou estratégias adoptadas durante o período de tempo fixo. Por tal razão, propõe-se o modelo para o tempo de resposta limitado.

Considere um conjunto de dados de tempos de resposta (RTs) para $I$ examinados em $J$ itens, todos os RTs são uma realização da variável aleatória $T_{i j}$, onde $i$ denota um examinado e $j$ um item. Define-se $d_{j}$ como o maior tempo utilizado pra solucionar o item $j$ e $c_{j}$ como o tempo mínimo. Então, é possível identificar a proporção do tempo (PT), $Z_{i j}$, que um examinado $i$ emprega no item $j$, a qual define-se da seguinte forma:

$$
Z_{i j}=\frac{T_{i j}-c_{j}}{d_{j}-c_{j}}
$$

Assim, $Z_{i j}$ esta no intervalo $<0,1>$ e pode ser modelado com uma distribuição limitada considerando parâmetros definidos como a seguir:

$$
\begin{gathered}
z_{i j} \mid \tau_{i}, \beta_{j}, \alpha_{j} \sim \pi\left(\theta_{i j}, \alpha_{j}^{2}\right) \\
g\left(\theta_{i j}\right)=\beta_{j}-\tau_{i}
\end{gathered}
$$



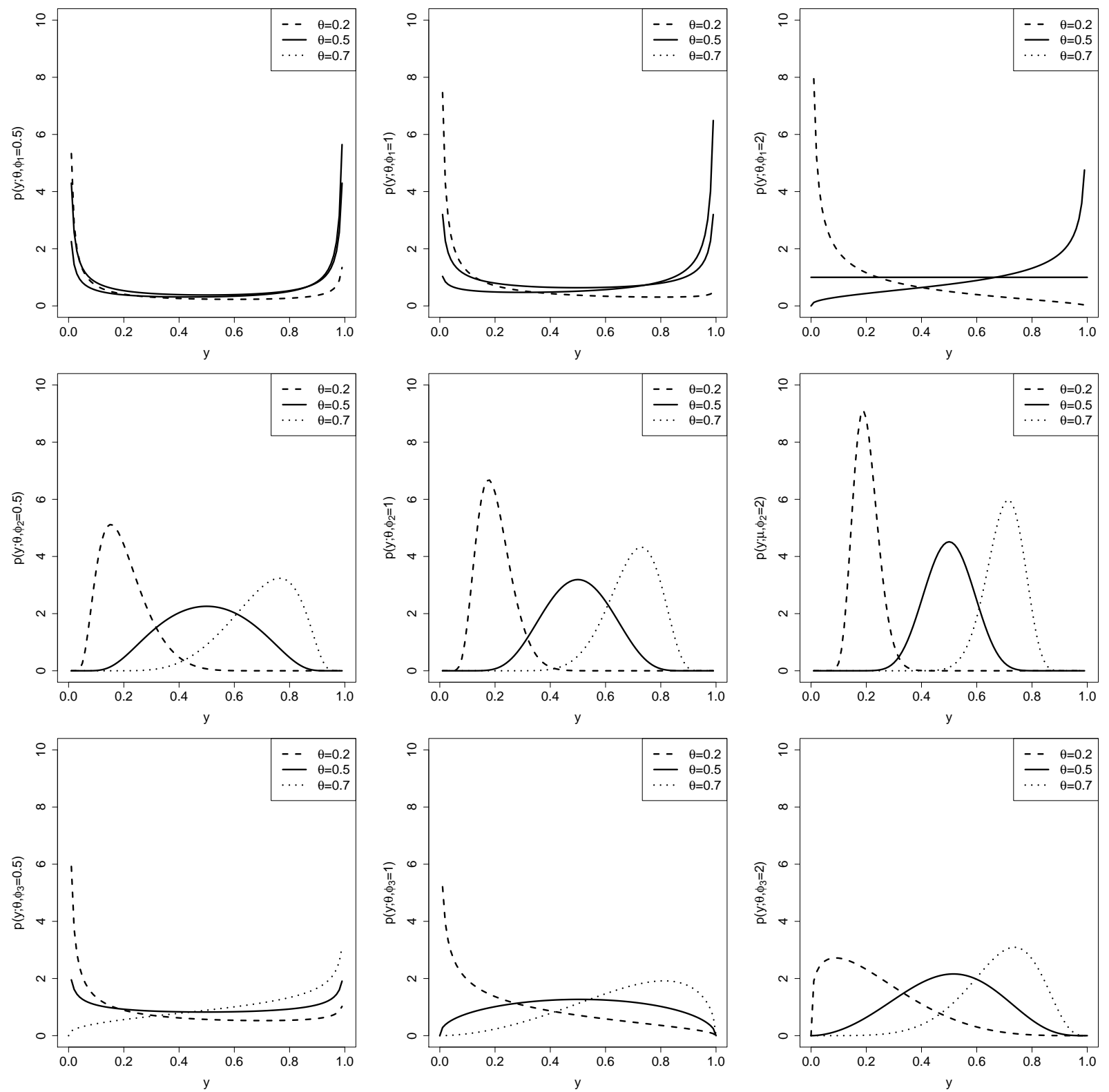

Figura 4.1: Função de densidade de probabilidade das distribuições limitadas beta, simplex e kumaraswamy com distintas quantidades da posição $\theta$ e a precisão $\phi$. 
onde $\pi(.,$.$) denota uma distribuição limitada, como definida na seção 4.2$, observe que $\alpha=\sqrt{\phi}$ pode ser interpretado como a precisão do tempo limitado dos avaliados no item $j . \beta_{j} \in \mathbb{R}$ define o parâmetro de posição do tempo limitado no item $j$ o qual pode ser interpretado como a intensidade do tempo limitado no item $j, \tau_{i} \in \mathbb{R}$ é o parâmetro de posição do examinado $i$, se ele é pequeno a proporção do tempo utilizado no item é maior e se ele é maior a proporção do tempo no item é menor, em consequência $\tau_{i}$ pode ser interpretado como a rapidez associada ao individuo avalidado. A função de ligação $g$ é definida como a função logit, isto é $g(x)=\log \left(\frac{x}{1-x}\right)$.

\subsection{Modelo hierárquico conjunto para o tempo de resposta limitado e a precisão (HBRT)}

Na presente seção, sugere-se um modelo conjunto para a rapidez, a qual tem um tempo limitado, e a precisão na resposta de um individuo avaliado. A proposta segue o trabalho do autor van der Linden (2007), mas considerando o tempo como sendo limitado. Para tal propósito, considere que o conjunto de dados o qual contém respostas de acerto, $y_{i j} \in\{0,1\}$, e proporções dos tempos de resposta, $z_{i j} \in<0,1>$, de um examinado $i$ no item $j$, são modelados de forma conjunta em dois níveis, da seguinte forma:

No primeiro nível, a rapidez e a precisão são modeladas utilizando duas distribuições. A precisão, resposta correta ou incorreta para cada um dos items, é modelada utilizando o modelo da TRI probito de dois parâmetros. Isto é,

$$
\begin{gathered}
y_{i j} \mid \zeta_{i}, a_{j}, b_{j} \sim \operatorname{Bernoulli}\left(p_{i j}\right) \\
p_{i j}=p\left(Y_{i j}=y_{i j} \mid \zeta_{i}, a_{j}, b_{j}\right)=\Phi\left(a_{j}\left(\zeta_{i}-b_{j}\right)\right)^{y_{i j}}\left(1-\Phi\left(a_{j}\left(\zeta_{i}-b_{j}\right)\right)\right)^{1-y_{i j}}
\end{gathered}
$$

onde a variável $Y_{i j}$ segue uma função de densidade de probabilidade Bernoulli com probabilidade de resposta correta definida como $p\left(Y_{i j}=1 \mid \zeta_{i}, a_{j}, b_{j}\right)=\Phi\left(a_{j}\left(\zeta_{i}-b_{j}\right)\right)$. Define-se o parâmetro de habilidade para o examinado $i$ como $\zeta_{i} \in \mathbb{R}$, ademais $a_{j}>0$ e $b_{j} \in \mathbb{R}$ simbolizam os parâmetros do item $j$ os quais são a discriminação e a dificuldade, respectivamente.

Por outro lado, a proporção do RT, $z_{i j}$, é modelada de acordo com a proposta apresentada na equação (4.4), onde a função de densidade de probabilidade da variável $Z$ segue uma distribuição limitada. Isto é,

$$
\begin{gathered}
z_{i j} \mid \tau_{i}, \beta_{j}, \alpha_{j} \sim \pi\left(\theta_{i j}, \alpha_{j}^{2}\right) \\
g\left(\theta_{i j}\right)=\beta_{j}-\tau_{i}
\end{gathered}
$$

onde $\pi$ simboliza uma distribuição limitada, a qual inclui a densidade da beta (4.1), simplex (4.2) ou kumaraswamy (4.3). A variável $\tau_{i} \in \mathbb{R}$ define o parâmetro de rapidez do examinado $i$, ademais os parâmetros dos itens, descritos na seção 4.3 , são simbolizados como $\alpha_{j}>0$ e $\beta_{j} \in \mathbb{R}$ os quais representam o poder de discriminação e da intensidade do tempo limitado no item $j$. A função de ligação $g$ se define como a função logit, isto é $g(x)=\log \left(\frac{x}{1-x}\right)$.

Como um segundo nível, assumindo independência condicional, das respostas dos examinados 
e itens, dados os parâmetros da habilidade e a rapidez, $\xi_{i}=\left(\zeta_{i}, \tau_{i}\right)$, e os parâmetros dos itens, $\nu_{j}=\left(a_{j}, b_{j}, \alpha_{j}, \beta_{j}\right)$, a distribuição conjunta toma a seguinte forma:

$$
p(\boldsymbol{y}, \boldsymbol{z} \mid \boldsymbol{\xi}, \boldsymbol{\nu})=\prod_{i=1}^{I} \prod_{j=1}^{J} p\left(y_{i j} \mid \zeta_{i}, a_{j}, b_{j}\right) p\left(z_{i j} \mid \tau_{i}, \alpha_{j}, \beta_{j}\right)
$$

onde $p\left(y_{i j} \mid.\right)$ e $p\left(z_{i j} \mid\right.$.) são definidas nas equações (4.5) e (4.4), respectivamente.

\subsection{Estimação Bayesiana}

Com o intuito de estimar os parâmetros do modelo conjunto proposto, sob a abordagem Bayesiana plena (ABP), maiores detalhes desta abordagem no capítulo 2.3, na presente seção definimos a função de verossimilhança, as distribuições a priori dos parâmetros do modelo e logo a distribuição a posteriori é obtida. A função de verossimilhança é definida mediante a seguinte equação:

$$
L(\boldsymbol{\xi}, \boldsymbol{\nu} \mid \boldsymbol{y}, \boldsymbol{z})=\prod_{i=1}^{I} \prod_{j=1}^{J} p\left(y_{i j} \mid \zeta_{i}, a_{j}, b_{j}\right) p\left(z_{i j} \mid \tau_{i}, \alpha_{j}, \beta_{j}\right) p\left(\xi_{i} \mid \mu_{\xi}, \Sigma_{\xi}\right) p\left(\nu_{j} \mid \mu_{\nu}, \Sigma_{\nu}\right)
$$

onde $\boldsymbol{\xi}=\left(\xi_{1}, \ldots, \xi_{I}\right)$ simboliza o vetor dos parâmetros da habilidade e rapidez, $\xi_{i}=\left(\zeta_{i}, \tau_{i}\right)$, para cada um dos examinados e $\boldsymbol{\nu}=\left(\nu_{1}, \ldots, \nu_{J}\right)$ é o vetor dos parâmetros do item, $\nu_{j}=\left(a_{j}, b_{j}, \alpha_{j}, \beta_{j}\right)$.

Assumindo $p\left(\xi_{i} \mid \mu_{\xi}, \Sigma_{\xi}\right)$ segue uma distribuição a priori bivariada Normal, define-se duas distribuições condicionais Normais marginais como a seguir: $p\left(\zeta_{i} \mid \mu_{\zeta}, \sigma_{\zeta}^{2}\right)$ e $p\left(\tau_{i} \mid \mu_{\tau}, \sigma_{\tau}^{2 c}\right)$, onde $\mu_{\tau}=\rho_{\zeta \tau} \zeta_{i}$ e $\rho_{\zeta \tau}$ proporcionam a relação entre $\zeta$ e $\tau$, ademais $\sigma_{\tau}^{2}=\sigma_{\tau}^{2 c}+\rho_{\zeta \tau}^{2}$, veja Fox et al. (2007). Adicionalmente, assume-se que o $v_{j}$ segue uma distribuição Normal multivariada com dimensão 4 e função de densidade definida como a seguir:

$$
p\left(\nu_{j} \mid \mu_{\nu}, \Sigma_{\nu}\right)=\frac{\left|\Sigma_{\nu}^{-1}\right|^{1 / 2}}{(2 \pi)^{5 / 2}} \exp \left\{-\frac{1}{2}\left(\nu_{j}-\mu_{\nu}\right)^{T} \Sigma_{\nu}^{-1}\left(\nu_{j}-\mu_{\nu}\right)\right\}
$$

a qual tem um vetor de médias definido como $\mu_{\nu}=\left(\mu_{a}, \mu_{b}, \mu_{\zeta}, \mu_{\tau}\right)$ e uma matriz de variâncias e covariâncias $\Sigma_{\nu}=\left[\begin{array}{cccc}\sigma_{a}^{2} & \sigma_{a b} & \sigma_{a \alpha} & \sigma_{a \beta} \\ \sigma_{b a} & \sigma_{b}^{2} & \sigma_{b \alpha} & \sigma_{b \beta} \\ \sigma_{\alpha a} & \sigma_{\alpha b} & \sigma_{\alpha}^{2} & \sigma_{\alpha \beta} \\ \sigma_{\beta a} & \sigma_{\beta b} & \sigma_{\beta \alpha} & \sigma_{\beta}^{2}\end{array}\right]$.

Com a finalidade de identificar o modelo conjunto, segundo van der Linden (2006, 2007), a média da rapidez é fixada como sendo $\mu_{\tau}=0$, a média da precisão e fixada como $\mu_{\zeta}=0$ e a variância da precisão também e fixada como sendo $\sigma_{\zeta}^{2}=1$. Para os parâmetros dos itens não são definidas restrições, pois a intenção é estimar os parâmetros em função aos dados de resposta.

Na presente seção assume-se a abordagem Bayesiana plena para estimar os parâmetros do modelo conjunto sugerido, pois nesta abordagem a conclusão em relação aos parâmetros é dada em termos de probabilidades as quais são condicionais aos dados observados, veja Gelman et al. (1995). Os modelos para a precisão e rapidez usualmente adotam a aproximação Bayesiana, veja por exemplo Im (2015); Klein Entink (2009); van der Linden (2007); Zhan et al. (2018). 
Dado que a distribuição dos parâmetros depende dos hiper-parâmetros, as seguintes distribuições a priori são definidas com a intenção de completar o modelo:

$$
\begin{gathered}
\rho_{\zeta \tau} \sim \mathcal{N}\left(0, \sigma_{\rho}^{2}\right) \\
\sigma_{\tau}^{2} \sim \operatorname{Inverse-Gamma}\left(v_{1}, v_{2}\right) \\
\Sigma_{\nu} \sim \text { Inverse-Whishart }\left(\Sigma_{\nu 0}^{-1}, v_{\nu 0}\right) \\
\mu_{\nu} \mid \Sigma_{\nu} \sim \operatorname{MVN}\left(\mu_{\nu 0}, \Sigma_{\nu}\right)
\end{gathered}
$$

$\sigma_{\rho}^{2}, v_{1}, v_{2}$ assume a quantidade 0.1 , outorgando escassa informação para o modelo. $\Sigma_{\nu 0}$ é uma matriz de identidade, $v_{\nu 0}=4$ e $\mu_{\nu 0}=(0,0,0,0)$, sugestões usadas a partir do trabalho de van der Linden (2007).

Assim, a distribuição a posteriori para os parâmetros define-se através da seguinte equação:

$$
p\left(\boldsymbol{\xi}, \boldsymbol{\nu}, \sigma_{\tau}^{2}, \rho_{\zeta \tau}, \boldsymbol{\mu}_{\nu}, \boldsymbol{\Sigma}_{\nu} \mid \boldsymbol{y}, \boldsymbol{z}\right) \propto p(\boldsymbol{y}, \boldsymbol{z} \mid \boldsymbol{\xi}, \boldsymbol{\nu}) p(\boldsymbol{\xi}, \boldsymbol{\nu}) p\left(\sigma_{\tau}^{2}\right) p\left(\rho_{\zeta \tau}\right) p\left(\mu_{\nu} \mid \boldsymbol{\Sigma}_{\nu}\right) p\left(\boldsymbol{\Sigma}_{\nu}\right)
$$

A implementação do modelo proposto utiliza o pacote R2WinBUGS do software R. Este pacote roda no software WinBUGS, o qual é uma versão interativa, no entorno Windows, do programa BUGS, para a análise de modelos estatísticos complexos utilizando as técnicas de Cadeias de Markov Monte Carlo (MCMC), veja Lunn et al. (2000). Os códigos para o modelo proposto são disponibilizados no apêndice F.

\subsection{Estudos de simulação}

Com o intuito de avaliar o desempenho dos modelos sugeridos apresenta-se nesta seção dois estudos de simulação, o primeiro esta en relação ao desempenho dos modelos da proporção do tempo de resposta, isto é o tempo limitado, utilizando as distribuições limitadas e o segundo estudo avalia a recuperação dos parâmetros no modelo hierárquico conjunto para o tempo limitado e a precisão.

\subsubsection{Recuperação dos parâmetros para o modelo do tempo de resposta limi- tado (BRT)}

Este primeiro estudo tem o propósito de conhecer o desempenho na recuperação dos parâmetros do modelo para o tempo de resposta limitado, utilizando duas distribuições limitadas a beta e a simplex. Conservando uma comparação com os parâmetros dos dados da aplicação, 25 conjuntos de dados do modelo BRT-beta foram gerados. Cada um contém 30 itens e 1000 examinados, para quantidades fixas de $\boldsymbol{\alpha}$ e $\boldsymbol{\beta}$, tais quantidades são semelhantes com os resultados obtidos para os dados da aplicação. As quantidades de $\boldsymbol{\tau}$ foram sorteados da distribuição Normal com média zero e desvío padrão de 0.5. Cada um dos conjuntos de dados replicados foram ajustados com o 


\begin{tabular}{ccccccc}
\hline Modelo & \multicolumn{3}{c}{ BRT-beta } & \multicolumn{3}{c}{ BRT-simplex } \\
\hline Parâmetro & RMSE & MAE & MT & RMSE & MAE & MT \\
\hline$\alpha$ & 0.2098 & 0.1828 & 47 & 0.0037 & 0.0030 & 41 \\
$\beta$ & 0.1189 & 0.1182 & 47 & 0.0606 & 0.0484 & 41 \\
\hline \hline
\end{tabular}

Tabela 4.1: Resultados do estudo de simulação do modelo BRT para as distribuições limitadas beta e simplex.

modelo BRT-beta. Análogamente, geramos 25 conjuntos de dados do modelo BRT-simplex, cada um inclui 1000 examinados e 30 itens, para quantidades fixas de $\boldsymbol{\alpha}$ e $\boldsymbol{\beta}$, similares às estimativas da aplicação, as quantidades $\boldsymbol{\tau}$ foram sorteadas da distribuição Normal com média zero e desvío padrão de 0.5. Cada um dos conjuntos de dados replicados foi ajustado com o modelo BRT-simplex.

Os resultados do processo de simulação detalhado previamente são avaliados utilizando a raiz do erro médio quadrático (RMSE), o erro médio absoluto (MAE) e o tempo médio de estimação em minutos (MT). Estas medidas de estimação são apresentadas na Tabela 4.1.

Podemos observar na Tabela 4.1 quantidades pequenas da RMSE e MAE sugerindo que a estimativa dos parâmetros é bastante próxima do parâmetro populacional e em consequência os modelos recuperam os parâmetros populacionais. Em caso da distribuição limitada beta é importante salientar que a estimativa do parâmetro $\alpha$ esta em uma escala muito diferente da simplex, o qual pode ser observado nas densidades da distribuição limitada apresentadas no apêndice B.

\subsubsection{Recuperação dos parâmetros no modelo hierárquico conjunto para o tempo limitado e a precisão}

Nesta subseção desenvolvemos um estudo de recuperação dos parâmetros para o modelo hierárquico conjunto do tempo limitado e a precisão (HBRT) considerando duas distribuições limitadas, a beta e a simplex. O propósito do presente estudo é conhecer características da recuperação dos parâmetros no modelo hierárquico conjunto. Em consequência geramos 10 conjuntos de dados do modelo HBRT-beta e cada um destes conjuntos de dados foi ajustado com este modelo. As quantidades dos parâmetros populacionales $\boldsymbol{\nu}=(\log (\boldsymbol{a}), \boldsymbol{b}, \log (\boldsymbol{\alpha}), \boldsymbol{\beta})$ foram sorteadas da distribuição Normal multivariada com vetor de médias $\mu_{\nu}=(-0.6,-1,3,-2)$ e matriz de covariâncias $\Sigma_{\nu}$, as quais conservam uma comparação com as estimativas obtidas na aplicação.

$$
\Sigma_{\nu}=\left[\begin{array}{cccc}
0.15 & 0 & 0 & 0 \\
0 & 1.7 & -0.4 & 0.2 \\
0 & -0.4 & 0.6 & -0.4 \\
0 & 0.2 & -0.4 & 0.4
\end{array}\right]
$$

Em relação à precição e rapidez limitada atribuidas aos examinados, as quantidades $\boldsymbol{\xi}=(\boldsymbol{\zeta}, \boldsymbol{\tau})$ foram sorteadas da distribuição Normal multivariada com vetor de média zero e matriz de covariância $\Sigma_{\xi}$

$$
\Sigma_{\xi}=\left[\begin{array}{cc}
1 & 0.05 \\
0.05 & 0.10
\end{array}\right]
$$




\begin{tabular}{lcccc}
\hline Modelo & \multicolumn{2}{c}{ HBRT-beta } & \multicolumn{2}{c}{ HBRT-simplex } \\
\hline Parâmetro & RMSE & MAE & RMSE & MAE \\
\hline $\mathrm{a}$ & 0.2288 & 0.2263 & 0.2012 & 0.1997 \\
$\mathrm{~b}$ & 0.2345 & 0.1909 & 0.4613 & 0.4605 \\
$\alpha$ & 0.4928 & 0.3970 & 0.0009 & 0.0007 \\
$\beta$ & 0.0298 & 0.0237 & 0.0269 & 0.0222 \\
$\rho_{\zeta \tau}$ & 0.0054 & 0.0041 & 0.0081 & 0.0068 \\
$\sigma_{\tau}^{2}$ & 0.0501 & 0.0497 & 0.0439 & 0.0436 \\
\hline
\end{tabular}

Tabela 4.2: Resultados da recuperação de parâmetros no modelo HBRT com diferentes distribuições limitadas

fixando assim os parâmetros $\rho_{\zeta \tau}=0.05$ e $\sigma_{\tau}^{2}=0.10$.

Adicionalmente, geramos 10 conjuntos de dados do modelo HBRT-simplex e cada um foi ajustado com o modelo HBRT-simplex. Preservando uma comparação com os dados da aplicação, os parâmetros populacionais foram sorteados da distribuição Normal multivariada com vetor de médias definido como $\mu_{\nu}=(-0.6,-0.6,-2.8,-2.1)$ e matriz de covariâncias

$$
\Sigma_{\nu}=\left[\begin{array}{cccc}
0.15 & 0 & 0 & 0 \\
0 & 0.5 & -0.2 & 0.2 \\
0 & -0.2 & 0.5 & 0 \\
0 & 0.2 & 0 & 0.3
\end{array}\right]
$$

Em relação aos examinados, fixamos a precisão e rapidez limitada $\boldsymbol{\xi}=(\boldsymbol{\zeta}, \boldsymbol{\tau})$ sorteando elas da distribuição Normal multivariada com vetor de média zero e matriz de covariância $\Sigma_{\xi}$

$$
\Sigma_{\xi}=\left[\begin{array}{cc}
1 & 0.05 \\
0.05 & 0.15
\end{array}\right]
$$

assim fixamos os parâmetros $\rho_{\zeta \tau}=0.05$ e $\sigma_{\tau}^{2}=0.15$.

Na tabela 4.2 apresentamos a média da raiz do erro quadrático médio (RMSE) e a média do erro médio absoluto (MAE) para os parâmetros dos itens e os parâmetros da relação entre a precisão e a rapidez limitada $\rho_{\zeta \tau}$ e a variância da rapidez limitada $\sigma_{\tau}^{2}$. Podemos notar que os erros para os parâmetros em relação ao tempo de resposta limitado $\alpha$ e $\beta$ alcançam quantidades bastante baixas com exceção do $\alpha$ no modelo HBRT-beta, o qual pode ser explicado pela escala do $\phi_{1}$ na distribuição limitada beta, a média dos parâmetros $\alpha_{j}$ foi fixada como $\exp (3)$ e no caso da simplex $\exp (-2.8)$, pois obter uma precisão adequada na beta se consegue com $\phi_{1}$ maior em relação ao $\phi_{2}$ da simplex, veja as densidades da beta no apêndice B. Em relação aos parâmetros $a$ e $b$ os quais são a dificuldade e discriminação do item no contexto da TRI, os resultados podem estar relacionados com o fato de modelar respostas de acerto $\{0,1\}$ o qual não consegue inserir uma variabilidade adequada para a quantidade de parâmetros os quais são estimados na TRI. 


\subsection{Aplicação}

Nesta seção ajustamos os modelos HBRT-beta e HBRT-simplex sugeridos na seção 4.4 utilizando o conjunto de dados da avaliação internacional PISA 2015, esta avaliação é aplicada em estudantes que estão finalizando estudos do ensino fundamental em 60 países ou economias. Para a aplicação da prova, um estudante utiliza um computador pessoal. O uso do computador tem a vantagem de registrar informação do desenvolvimento da prova e fornece a opção de obter o tempo de resposta de cada estudante para cada um dos itens.

Os dados os quais são parte de esta análise correspondem a 28 itens de leitura, os quais são respostas dos clusters R1 e R3 do "bookid" 37, veja OECD (2017) página 38. Respostas completas dos clusters R1 e R3 foram selecionadas com o intuito de evitar os dados omissos. Cada cluster foi desenhado para estarem concluídos em 30 minutos e depois de dois clusters os estudantes têm uma pausa de 10 minutos. Assim, o tempo utilizado em dois clusters não precisaria mais de uma hora. Alguns registros, os quais tomam mais de 70 minutos, foram considerados como problemas na aplicação e foram excluídos da análise. Um total de 4960 tempos de resposta (RTs) e respostas de acerto, de 28 itens, em 53 países ou economias, foram utilizados na presente aplicação dos modelos.

O tempo de resposta limitado, isto é, a proporção do RT para um examinado $i$ no item $j$, $z_{i j}$, foi calculado utilizando a transformação sugerida na seção 4.3 , isto é $z_{i j}=\frac{t_{i j}}{d_{j}+1}$, onde $d_{j}$ é o maior tempo em que um examinado contesta o item $j$ e adicionamos 1 com a intenção de evitar uma proporção de 1. Esta variável, $z_{i j}$, pode ser interpretada como a proporção do tempo conseguida pelo examinado $i$ em relação ao maior tempo utilizado no item $j$. Um valioso atributo desta transformação é que se preserva o comportamento assimétrico do tempo como podemos observar na Figura 4.3 em relação à Figura 4.2. A média e o desvio padrão relacionados com cada um dos itens são ilustradas na Figura 4.4, podemos observar a existência de uma correlação positiva entre a média e o desvio padrão, isto é, um item com maior tempo limitado médio está associado com maior variabilidade do tempo limitado.

Em relação às respostas de exatidão ou precisão dos itens, $y_{i j}$, temos que três itens, dos 28 , apresentam crédito parcial. Tais itens foram transformados como sendo de resposta correta e incorreta, onde o crédito parcial é uma resposta incorreta. A Figura 4.4 ilustra a média da exatidão, isto é a proporção de acerto para cada item e o desvio padrão associado. Podemos observar que uma variabilidade maior é atingida com uma proporção de acerto de 0.5 .

O conjunto de dados do tempo limitado de resposta $\boldsymbol{z}$ e precisão da resposta $\boldsymbol{y}$ foi ajustado utilizando o modelo HBRT-beta e HBRT-simplex, propostos na seção 4.4, o ajuste segue o método de estimação apresentado na seção 4.5. A implementação dos modelos utilizou 16000 iterações, 4000 delas foram consideradas como aquecimento (burning) e para evitar autocorrelação seleções sistemáticas de quatro foram tomadas para completar a amostra. O ajuste do modelo HBRT-beta foi realizado em seis horas e treinta minutos aproximadamente e para o modelo HBRT-simplex tomou nove horas. A média a posteriori para os parâmetros dos itens associados ao componente da TRI, isto é a dificuldade e discriminação dos itens, e ademais os associados ao componente do tempo limitado de resposta (BRT) para os modelos ajustados são ilustradas na Figura 4.5. 


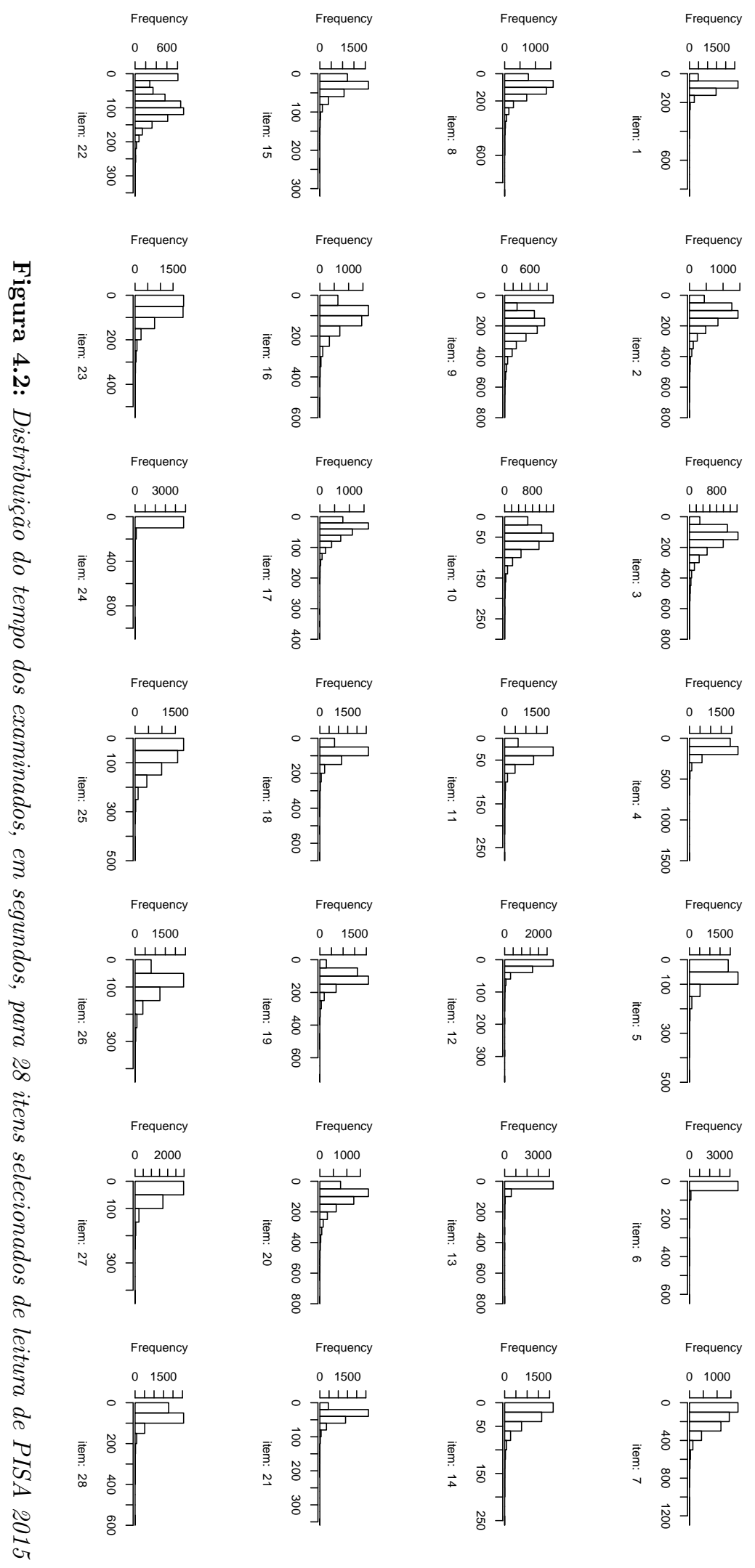



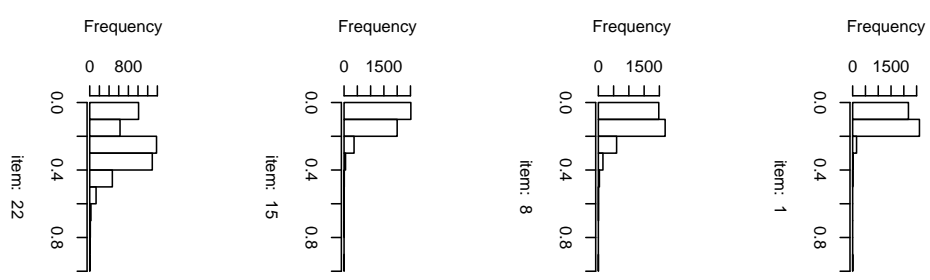

Frequency

Frequency
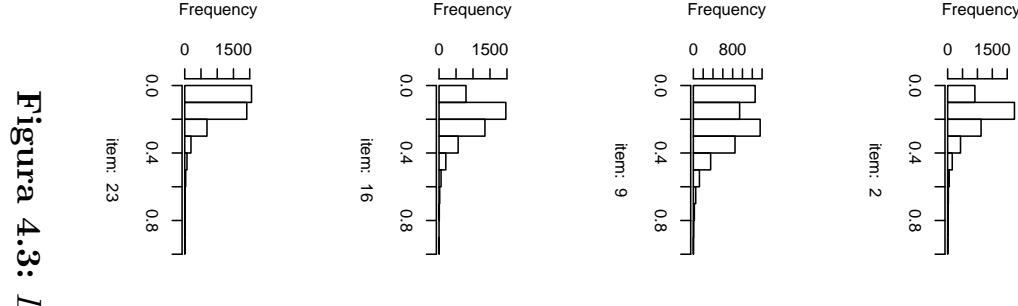

2. $\quad$ Frequency

Frequency

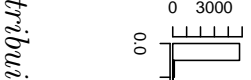
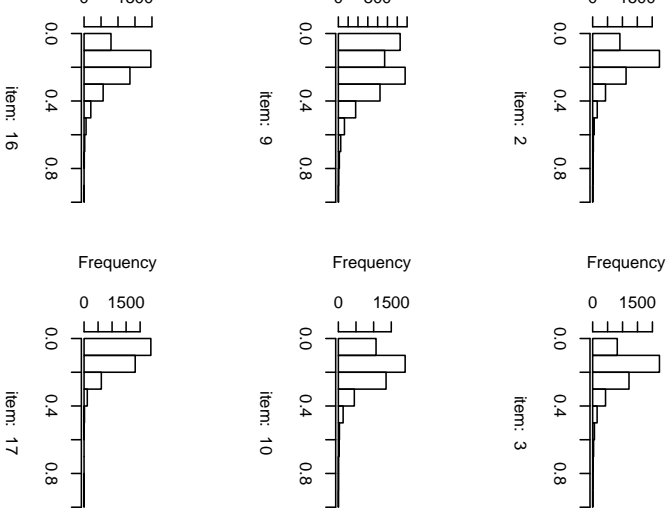

$\stackrel{8}{8}$

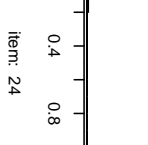

Frequency

ה.
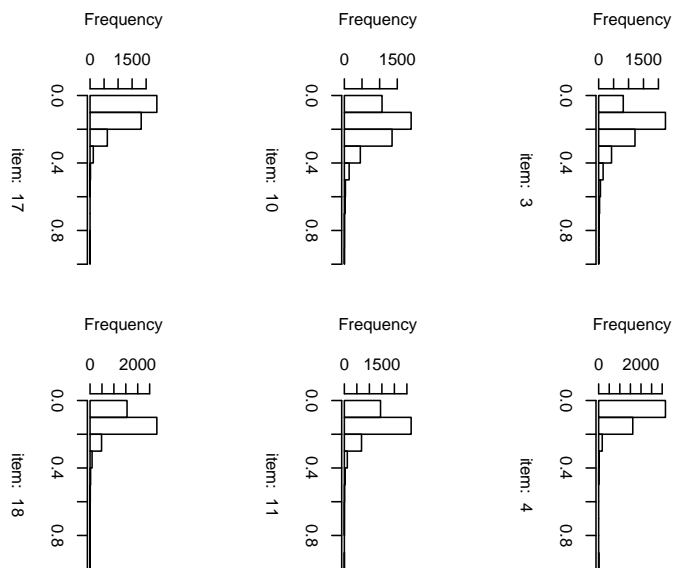

Frequency

$0 \quad 1500$

Frequency

के

5
3
$\infty$
3

$\infty$

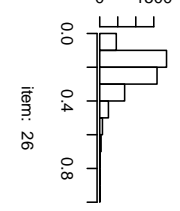

02000
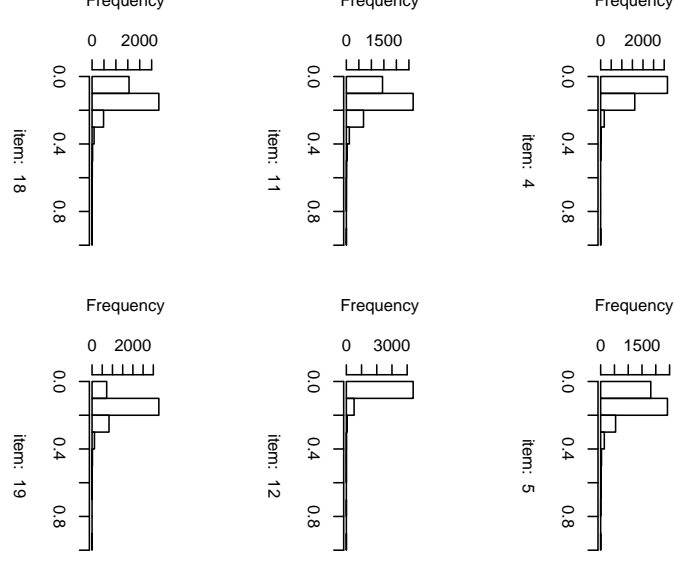

Frequency

Frequency
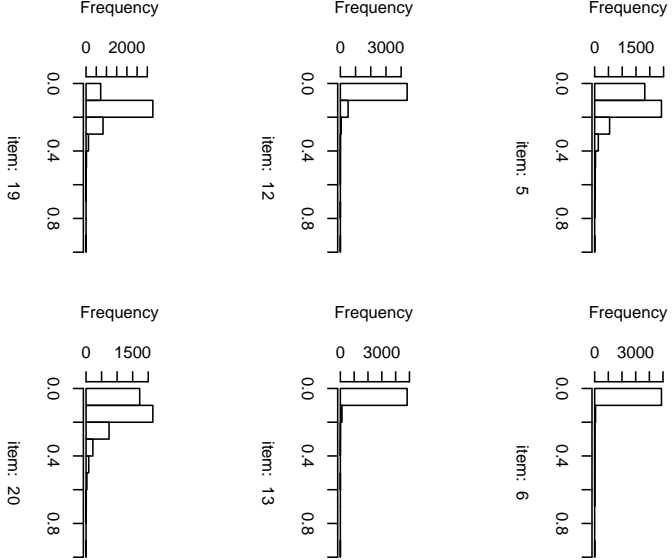

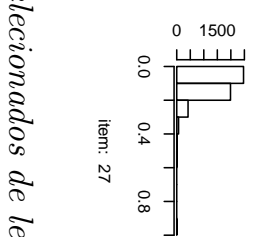

हू.

ह 01500

Frequency

$0 \quad 1500$
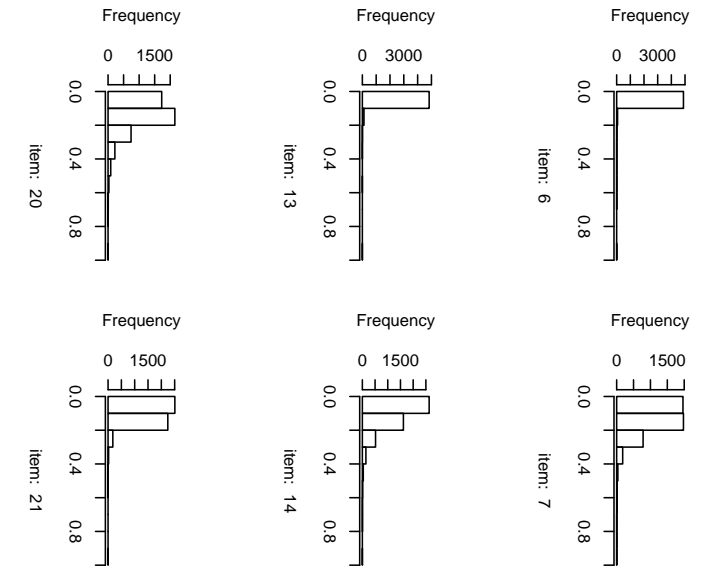

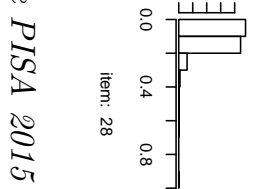

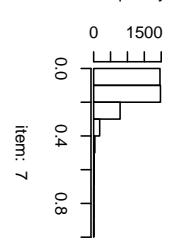



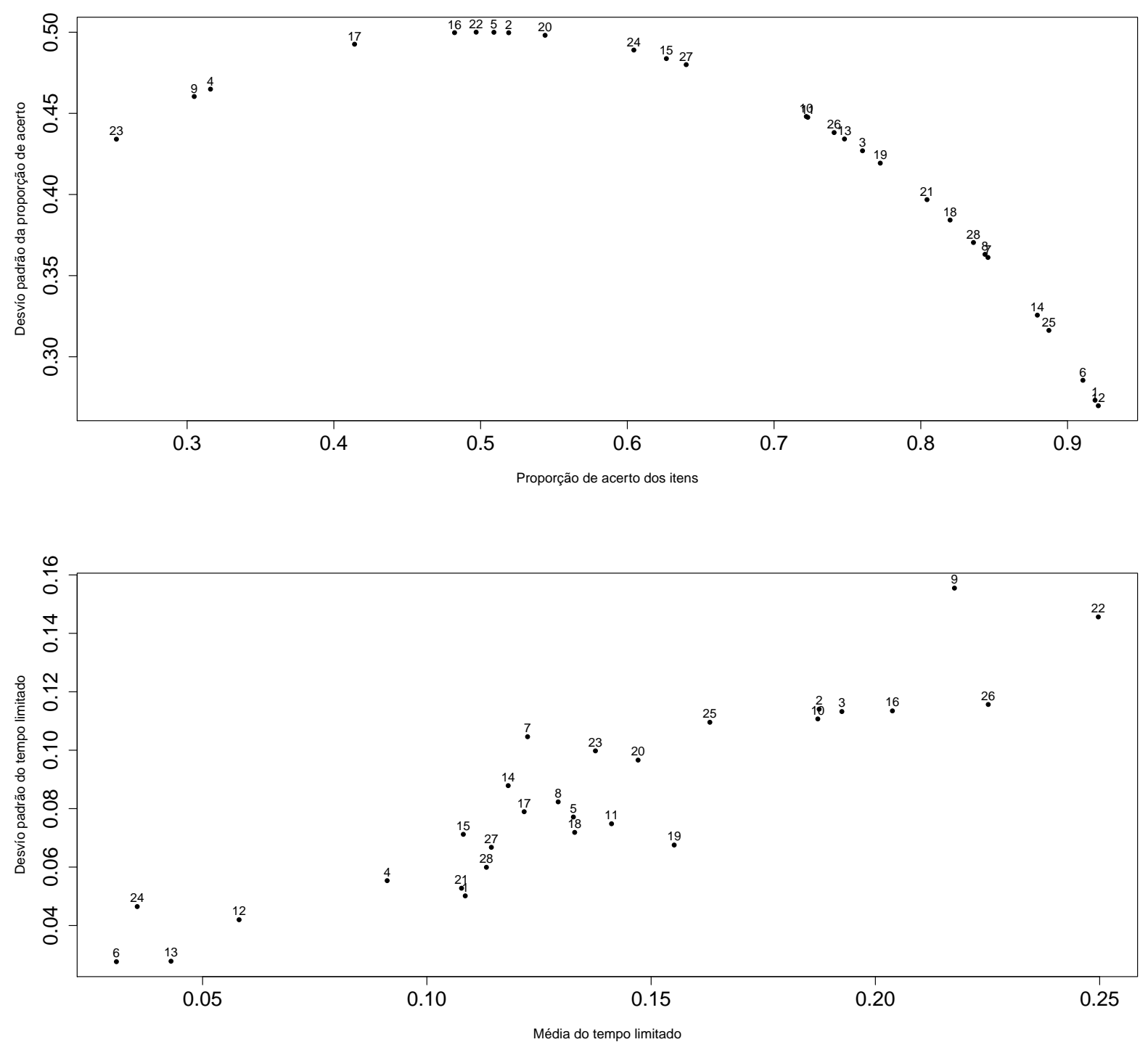

Figura 4.4: Média e desvío padrão das respostas de acerto (exatidão) e tempo limitado para cada um dos 28 itens de leitura selecionados do PISA 2015 

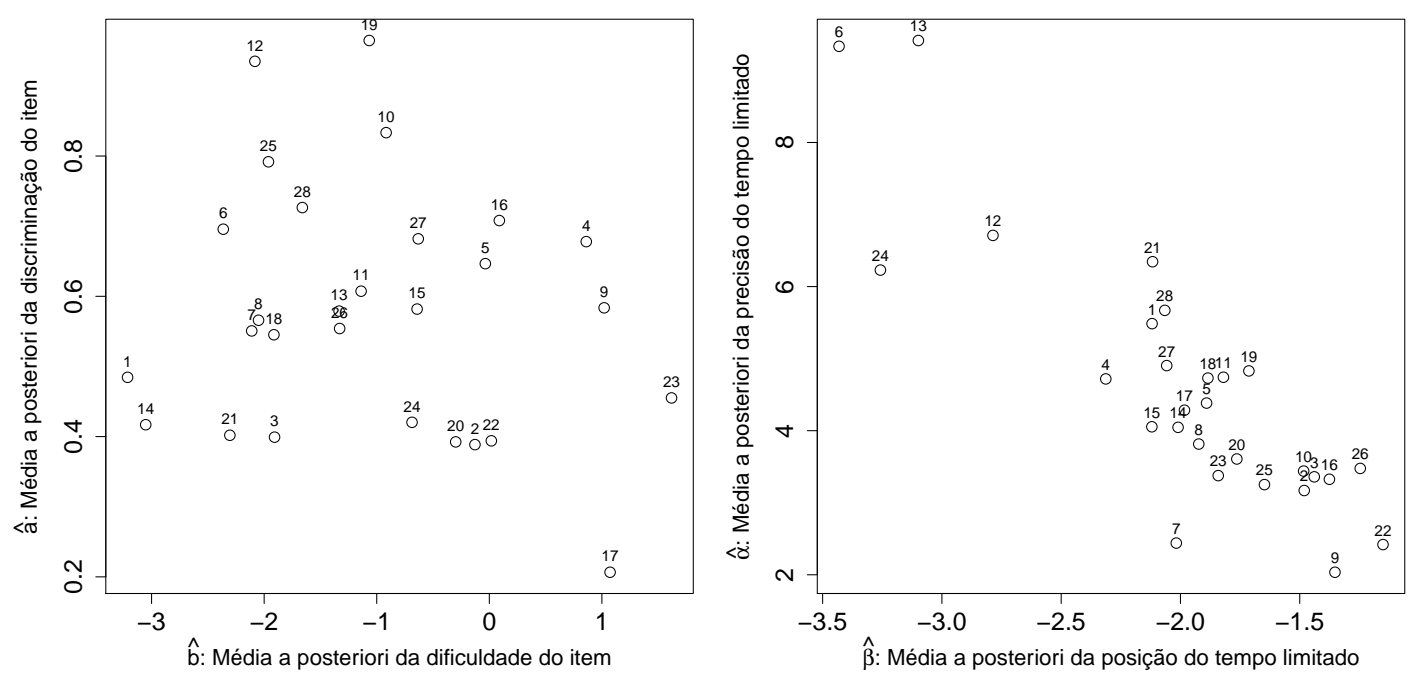

Figura 4.5: (a) Estimativa dos parâmetros dos items na TRI e (b) Estimativa dos parâmetros dos items no BRT para cada um dos 28 itens de leitura aplicados em PISA 2015, usando o modelo HBRT-beta.

Em relação ao modelo ajustado HBRT-beta, da Figura 4.5 podemos observar que a estimação dos parâmetros associados ao BRT conserva relação com a média e a variância apresentada na Figura 4.4, por exemplo, em cada item, a posição do tempo limitado $\beta$ é semelhante à média do BRT, a precisão do tempo limitado $\alpha$ é o inverso da variância do BRT. No caso dos parâmetros do componente da TRI observamos que a proporção de acerto é próxima à dificuldade para alguns itens, especificamente para os itens com dificuldades extremas (itens 1 e 23) e não observamos uma relação clara do parâmetro de discriminação com a variabilidade da proporção de acerto. Além disso, a correlação média e variância para o tempo limitado é preservada nos parâmetros associados ao BRT.

A média a posteriori do parâmetro $\rho_{\zeta \tau}$ é 0.0169 , a qual tem uma interpretação da relação de "trade-off" entre a rapidez limitada $\tau$ e a precisão na resposta $\zeta$, podemos concluir que não ha uma relação, ao nível geral, da rapidez limitada e a precisão. A Figura 4.7 apresenta as estimativas para cada um dos avaliados, podemos observar que alguns avaliados com maior rapidez são bastante precisos, porém a maioria dos avaliados têm distintos níveis de precisão com similar rapidez. As relações entre os parâmetros dos itens podem ser observadas na estimativa da matriz $\Sigma_{\nu}$.

$$
\hat{\Sigma_{\nu}}=\left[\begin{array}{cccc}
0.155 & -0.088 & 0.046 & -0.022 \\
-0.088 & 1.707 & -0.372 & 0.212 \\
0.046 & -0.372 & 0.601 & -0.368 \\
-0.022 & 0.212 & -0.368 & 0.384
\end{array}\right]
$$

Em relação ao modelo ajustado HBRT-simplex, note-se na Figura 4.6 a estimação dos parâmetros associados ao BRT e os associados ao componente da TRI. Se observa que a relação com a média e a variância apresentada na Figura 4.4 não é conservada totalmente, pois no caso dos itens $6,12,13$ e 24 a média posteriori da precisão do tempo limitado $\alpha$ nestes itens é baixa, assim não observamos uma relação entre a posição e a precisão do tempo limitado, assim no modelo simplex a variância não esta relacionado de forma direita com a precisão. No caso dos parâmetros do componente da 

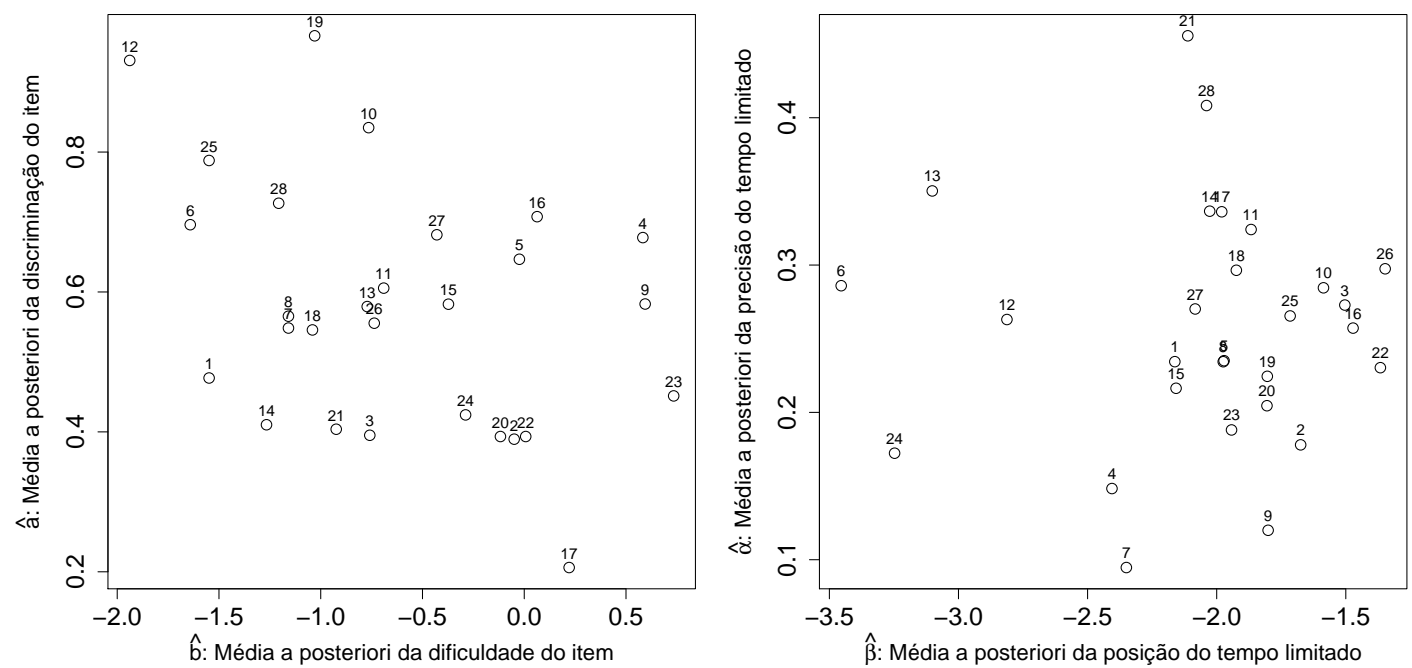

Figura 4.6: (a) Estimativa dos parâmetros dos items na TRI e (b) Estimativa dos parâmetros dos items no BRT para cada um dos 28 itens de leitura aplicados em PISA 2015, usando o modelo HBRT-simplex.

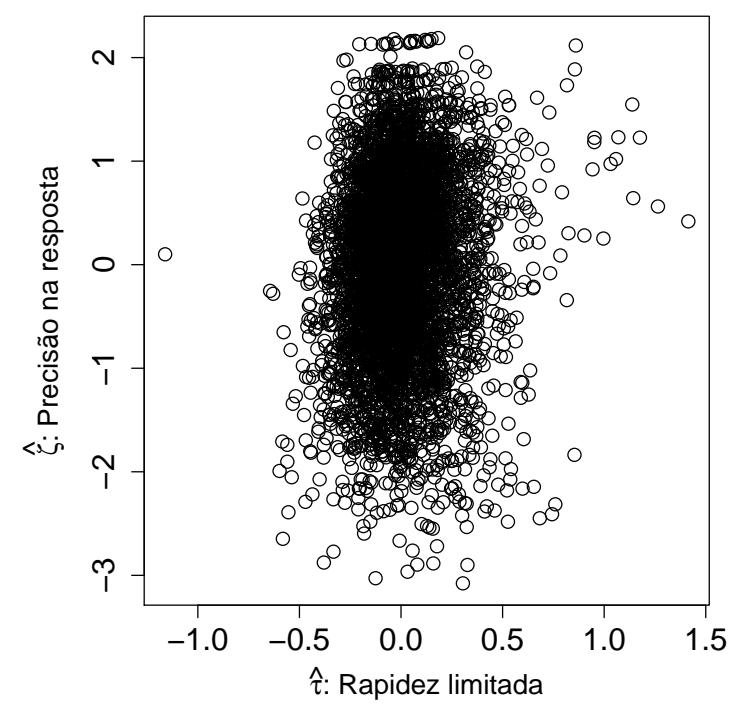

Figura 4.7: Relação entre a rapidez limitada e a precisão na resposta para os avaliados em leitura em PISA 2015, usando o modelo HBRT-beta. 


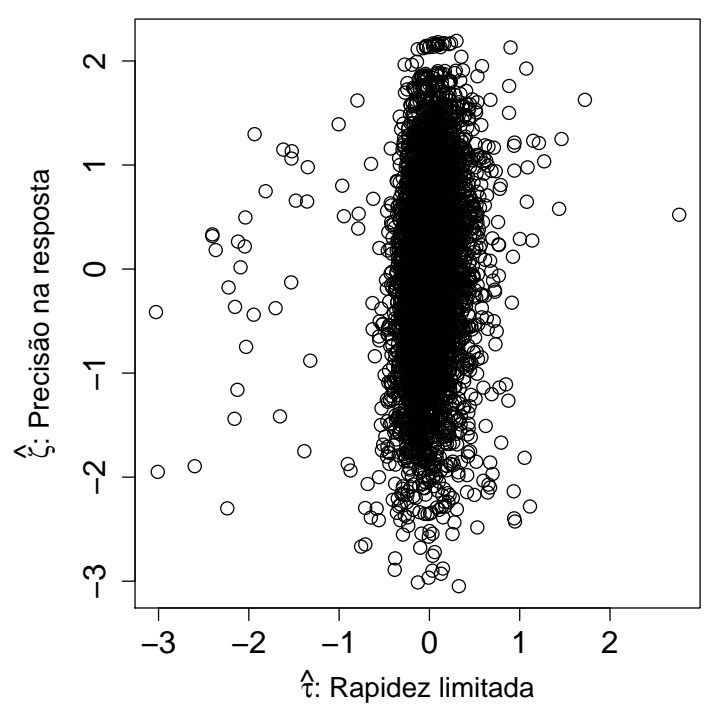

Figura 4.8: Relação entre a rapidez limitada e a precisão na resposta para os avaliados em leitura em PISA 2015, usando o modelo HBRT-beta.

TRI observamos que a proporção de acerto é próxima à dificuldade so para os itens com dificuldades maiores (itens 4, 9 e 23) e de forma similar ao modelo beta não observamos uma relação clara do parâmetro de discriminação com a variabilidade da proporção de acerto.

No modelo HBRT-simplex, a média a posteriori do parâmetro $\hat{\rho}_{\zeta \tau}=0.0380$, a qual sugere, da mesma forma que o modelo HBRT-beta, que não existe uma relação, de forma geral, entre a rapidez limitada $\tau$ e a precisão na resposta $\zeta$. De forma individual, podemos observar na Figura 4.8 as estimativas da rapidez limitada e da precisão para cada um dos avaliados, note que alguns avaliados com menor rapidez tem precisões baixas, porém a maioria dos avaliados têm distintos níveis de precisão com similar rapidez. A seguir apresentamos a estimativa da matriz $\Sigma_{\nu}$ para este modelo, onde podemos observar as relações entre os parâmetros dos itens. Como foi analizado previamente, de forma contraria ao modelo HBRT-beta, não se observa uma relação entre a posição e precisão do tempo limitado.

$$
\hat{\Sigma_{\nu}}=\left[\begin{array}{cccc}
0.157 & -0.092 & -0.010 & -0.023 \\
-0.092 & 0.570 & -0.202 & 0.113 \\
-0.010 & -0.202 & 0.540 & 0.015 \\
-0.023 & 0.113 & 0.015 & 0.333
\end{array}\right]
$$

\subsection{Comentários finais}

No presente capítulo, as distribuições limitadas foram utilizadas para modelar o tempo limitado, isto é a proporção do tempo de resposta ao item para um avaliado em relação ao maior tempo obtido no item, como uma forma alternativa aos modelos tradicionais de respostas relacionadas com o tempo, tais modelos tradicionais consideram que o tempo de resposta na prova é ilimitado e utilizam o modelo Lognormal, mas o tempo na prova é limitado. O desempenho dos modelos sugerido, o qual aborda o tempo de resposta como sendo limitado é satisfatorio como foi analizado nos estudos de simulação. 
Adicionalmente, sugerimos os modelos hierárquicos do tempo de resposta limitado (HBRT), o qual modela o tempo de resposta limitado e a resposta ao item de forma conjunta, onde ademais são modeladas as relações de ambos constructos (a precisão e a rapidez limitada).

Os estudos de simulação sugerem uma boa performance do modelo HBRT sendo uma alternativa ao modelo hierárquico proposto pelo autor van der Linden (2007). Adicionalmente, a aplicação desenvolvida mostra várias posibilidades do modelo proposto no ambito das Avaliações. Trabalhos futuros podem ser desenvolvidos utilizando distribuições assimétricas para a precisão e a rapidez. 


\section{Capítulo 5}

\section{Conclusões}

Modelos estatísticos para respostas limitadas e respostas de exatidão ou precisão as quais contém estruturas relacionadas ou dependentes foram desenvolvidos ao longo do presente trabalho. A motivação principal, em cada modelo, foi incluir características existentes nos dados as quais geram relações de dependência.

Nos modelos apresentados, as relações presentes nos dados são modeladas através de efeitos aleatórios com características específicas. Tais efeitos aleatórios conseguem modelar relações de diversas estruturas tais como relações geradas pela composição de testlets em provas de compreensão leitora ou estruturas de relações espaciais presentes em unidades as quais têm uma localização espacial. Ambas as situações precisam que a estrutura de relações presentes nos dados seja incluída no modelo através de um vetor ou uma matriz a qual contém as relações a serem modeladas. Tais situações supõem que o efeito aleatório pode ser modelado utilizando propriedades da distribuição Normal.

Nesse contexto, para o caso das relações presentes em testlets, define-se um efeito aleatório unidimensional para cada um dos testlets presentes na prova. Tal efeito aleatório unidimensional encapsula a relação das respostas dos itens os quais compõem um testlet. Este efeito reúne diferenças das respostas ao interior do testlet como sendo aleatórias normais com média zero. Assim, se tais quantidades são distintas para todos os examinados avaliados o efeito terá uma variância maior. Tal variância do efeito testlet é interpretada como uma medição do efeito testlet.

Com a intenção de modelar as relações espaciais presentes nos dados define-se um vetor aleatório como sendo um processo de Markov gaussiano intrínseco (Intrinsec gaussian Markov random field - IGMRF) o qual consegue encapsular as relações através do inverso da matriz de variânciacovariância da distribuição Normal multivariada. Esta matriz é chamada como uma matriz de precisão $\boldsymbol{R}$ a qual inclui as relações presentes nos dados considerando a definição das distribuições condicionais completas de cada uma das marginais da Normal multivariada. Ademais, as relações nos dados podem ser incluídas através da mesma matriz de variância-covariância considerando o modelo Leroux . Assim, as relações de localização espacial são modeladas mediante o efeito aleatório espacial, tal efeito é um vetor que segue uma distribuição Normal multivariada com uma matriz de variância-covariância a qual inclui as relações presentes no conjunto de dados modelado. 
Em relação às respostas limitadas, temos que o tempo de resposta na prática não é infinito, assim define-se o tempo em termos da proporção do tempo utilizado na resolução de cada item presente na prova, esta variável resposta da proporção do tempo é limitada no intervalo unitário. Da mesma forma que as variáveis definidas como proporções ou taxas as quais estão encaixadas no intervalo unitário. Esta característica das variáveis limitadas no intervalo $(0,1)$ é modelada através de distribuições com suporte no intervalo unitário. Assim, se modelou no capítulo 4 o tempo de resposta limitado (BRT) considerando a característica do tempo que é limitada aplicando as distribuições limitadas Beta e Simplex, com suporte no intervalo $(0,1)$. Porém, pode-se utilizar também a distribuição Kumaraswamy como foi aplicado, no contexto de regressão, para respostas de proporções no capítulo 3. Ademais, o modelo BRT proposto pode ser modelado de forma conjunta às respostas aos itens de uma prova sugerindo desta forma o modelo hierárquico conjunto para o tempo de resposta limitado e a precisão (HBRT). O modelo considera que o tempo de resposta pode estar relacionado com a habilidade de um examinado, mas é uma característica distinta, própria do individuo, a qual é modelada como um traço latente particular chamado rapidez. As respostas de precisão, corretas ou incorretas, geram o traço latente conhecido como a habilidade da pessoa no construto. O modelo inclui a estimação da correlação entre a rapidez e a habilidade, na aplicação se estimou esta relação, sendo muito baixa, o qual demostra que ambos os traços, de forma geral, não tem uma relação categórica. Ao nível do examinado, temos pessoas com uma rapidez similar têm distintas habilidades.

Os tempos limitados conjuntamente com as respostas aos items de uma prova sugerem o desenvolvimento do modelo geral hierárquico testlet conjunto para o tempo limitado e a precisão (HTBRT), o qual esta sendo analisado e escrito. Tal modelo consegue incluir as relações de dependência presente nas respostas com estruturas testlet e modela de forma conjunta os tempos de resposta limitados e as respostas de exatidão dos itens de uma prova.

A estimação dos parâmetros para os modelos apresentados na presente tese foi sob a abordagem Bayesiana. Exploramos uma forma de inferência, no contexto Bayesiano, usando aproximações aninhadas da integral de Laplace (INLA) a qual reduz significativamente o tempo computacional. Porém, ainda temos o desafío na estimação dos modelos sugeridos. Pois, a estimação para modelos com efeitos aleatórios particulares é demorado. O modelo geral HTBRT para um conjunto de 4960 respostas em 28 itens demorou 16 horas.

\subsection{Desenvolvimentos futuros}

- Estudos para aprimorar o tempo na estimação dos modelos apresentados, especificamente do capítulo 4, podem ser diretamente analisados em estudos posteriores.

- O modelo do tempo de resposta limitado, BRT, pode ser ampliado utilizando a distribuição Kumaraswamy.

- O modelamento de um construto latente contínuo no intervalo unitário pode ser desenvolvido de forma direita utilizando as distribuições limitadas estudadas na presente tese. 


\section{Apêndice A}

\section{O software STAN}

STAN é uma linguagem de programação probabilística para especificar modelos estatísticos e fazer inferências desde a perspectiva Bayesiana, veja Carpenter et al. (2017). STAN define o log da função de densidade de probabilidade para os parâmetros de interesse condicionados ao conjunto de dados e fornece a estimação a posteriori dos parâmetros considerando cadeias de markov Monte Carlo (MCMC). Estas cadeias têm uma variação ao tradicional método de Metropolis Hastings, STAN considera o algoritmo No-U-Turn sampler (NUTS), este algoritmo é uma adaptação do amostrador Hamiltoniano Monte Carlo (HMC). Na presente seção define-se o algoritmo HMC e a variação no algoritmo NUTS usado no software STAN.

\section{A.1 Algoritmo Hamiltoniano Monte Carlo (HMC)}

Geralmente na inferência Bayesiana definimos uma distribuição a priori do parâmetro de interesse como $p(\theta)$ e a distribuição a posteriori é definida como $p(\theta \mid \boldsymbol{y})$. O algoritmo HMC incorpora uma variável adicional de momento $\rho$, na distribuição a posteriori, e utiliza a seguinte densidade conjunta para a extração de amostras:

$$
p(\rho, \theta)=p(\rho \mid \theta) p(\theta)
$$

onde a densidade da variável auxiliar de momento é uma Normal multivariada, a qual não depende do parâmetros $\theta$, assim $\rho \sim \mathcal{N}_{m}(0, M)$.

A matrix $M$ é como uma transformação do espaço paramêtrico a qual faz a amostragem mais eficiente, veja Betancourt (2017) citado no manual de usuario do STAN. O software STAN fixa $M^{-1}$ como sendo a diagonal estimada da covariança a qual é obtida no proceso de aquecimento.

Define-se o Hamiltoniano $H$ como a densidade conjunta $p(\rho, \theta)$ da seguinte forma:

$$
\begin{aligned}
H(\rho, \theta) & =\log p(\rho, \theta) \\
& =-\log (\rho \mid \theta)-\log (\theta) \\
& =T(\rho \mid \theta)+V(\theta)
\end{aligned}
$$

O algoritmo começa com valores iniciais do parâmetro $\theta$, a mudança para um novo valor implica duas etapas. Primeiro, geramos um valor da variável de momento $\rho \sim \mathcal{N}_{m}(0, M)$. Logo, a densi- 
dade $p(\rho, \theta)$ actualiza o valor do parâmetro $\theta$ e o novo $\rho$ é obtido com a solução de duas equações diferenciais. Este proceso é realizado mediante o algoritmo de integração numérica conhecido como "leapfrog integrator" o qual se adapta com a intenção de obter resultados estáveis para o sistema de equações hamiltonianas.

O algoritmo leapfrog considera pasos discretos de um intervalo pequenho de tempo $\epsilon$ e inicia o algoritmo extraindo um valor para a variável momento $\rho \sim \mathcal{N}_{m}(0, M)$ de forma independênte ao valor de $\theta$, logo actualiza-se a metade da variável momento e completamente a variável de posição $\theta$, da seguinte forma:

$$
\begin{aligned}
& \rho \leftarrow \rho-\frac{\epsilon}{2}, \frac{\partial V}{\partial \theta} \\
& \theta \leftarrow \theta+\epsilon M^{-1} \rho \\
& \rho \leftarrow \rho-\frac{\epsilon}{2}, \frac{\partial V}{\partial \theta}
\end{aligned}
$$

O algoritmo leapfrog se aplica $L$ vezes, com o qual obtemos uma amostra simulada de $L \times \epsilon$. O estado ao final das $L$ repetições é denotada como $\left(\rho^{*}, \theta^{*}\right)$. Avalia-se a aceitação o não da proposta $\left(\rho^{*}, \theta^{*}\right)$ mediante a fase de aceitação de Metropolis onde a probabilidade de aceitar a proposta é definida como: $\min \left(1, \exp \left(H(\rho, \theta)-H\left(\rho^{*}, \theta^{*}\right)\right)\right)$. Se não fosse aceita a proposta, o valor previo é retornado para a seguinte iteração e é utilizado como valores de inicialização.

O algoritmo HMC considera três parâmetros, os quais são fixados ao inicio do algoritmo: $\epsilon$, M e $L$. Na pratica, a eficiencia amostral, em termos da velocidade das iterações e da quantidade das iterações para obter uma amostra efetiva, é bastante sensível à escolha dos três parâmetros. A seguir apresentamos o algoritmo NUTS o qual elimina a neccesidade da escolha de $L$.

\section{A.2 O algoritmo NUTS}

O algoritmo no-U-turn sampler (NUTS) seleciona de forma automática uma quantidade adequada de passos leapfrog, $L$, em cada iteração com a inteção de percorrer a posteriori sem a neccesidade de fazer trabalho excessivo. A ideia é conhecer quando a simulação da proposta $\theta^{*}$ não é muito distante do valor inicial $\theta$, veja Hoffman e Gelman (2014).

NUTS gera uma proposta considerando como ponto inicial o parâmetro da ultima iteração. Logo gera um vetor de momento aleatório, o qual muda o sistema inicial com passos em frente e para trás ("forward" e "backward") ao mesmo tempo, construindo assim uma árvore balanceada binaria. Em cada iteração do algoritmo NUTS a profundidão da árvore é incrementada em 1, duplicando a quantidade de passos leapfrogs, consequentemente duplicando o tempo efetivo computacional. No STAN o algoritmo finaliza com uma das duas condicões:

- O critério NUTS é satisfeito. Isto é um ciclo-U ("U-turn") no espaço Euclidiano é gerado em uma subárvore para uma subárvore nova ou uma árvore completa.

- A profundidade da árvore completa atinge a máxima permitida. 
A quantidade de passos leapfrog é acotada por $2^{d}-1$, onde $d$ é a máxima profundidade. No STAN, ambos a profundidade da árvore e a quantidade de passos leapfrog calculados são reportados junto com as estimativas dos parâmetros.

STAN permite a eleção entre três opções da métrica Euclidiana $M$. As quais são as seguintes:

- uma métrica unitaria (matriz diagonal de ums)

- uma métrica diagonal (matriz diagonal com quantidades específicas), e

- uma métrica densa (matriz simétrica, densa e definida positiva)

Na métrica diagonal, as variâncias são estimadas baseadas nas iterações na etapa do aquecimento. Se a métrica é densa se estima covariâncias as quais regulam a matriz diagonal estimada.

Finalmente, define-se o tempo de integração como uma função da quantidade de passos, isto é, $L \epsilon$. STAN fixa o tempo aproximado de integração e o tamano dos passos $\epsilon$. 
APÊNDICE A 


\section{Apêndice B}

\section{Densidades da distribuição limitada}

As figuras B.1, B.2 e B.3 retratam densidades adicionais às apresentadas no capítulo 3 para a distribuição limitada com diferentes quantidades de $\theta$ e $\phi$. 

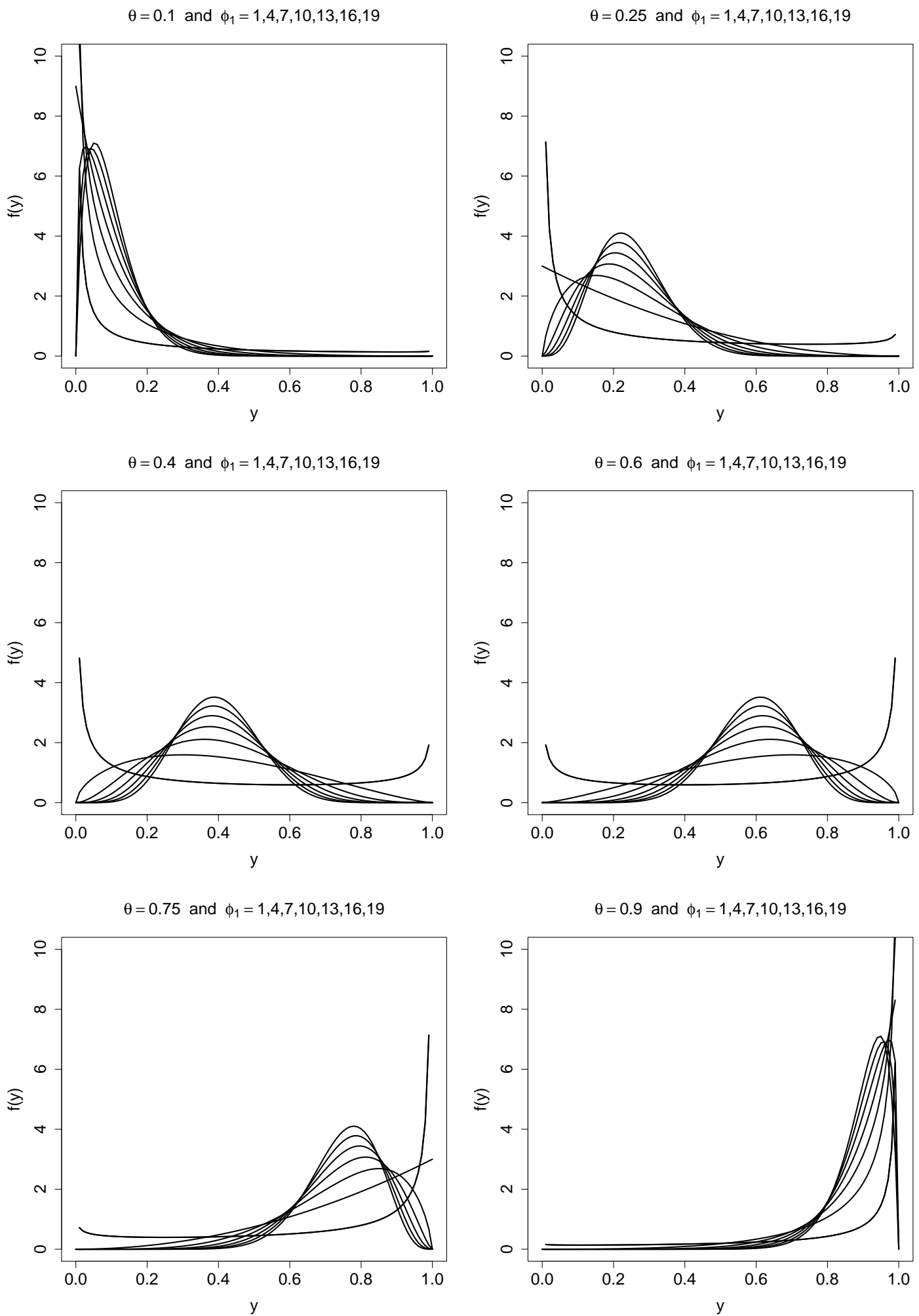

Figura B.1: Função de densidade de probabilidade para a ditribuição limitada Beta. 

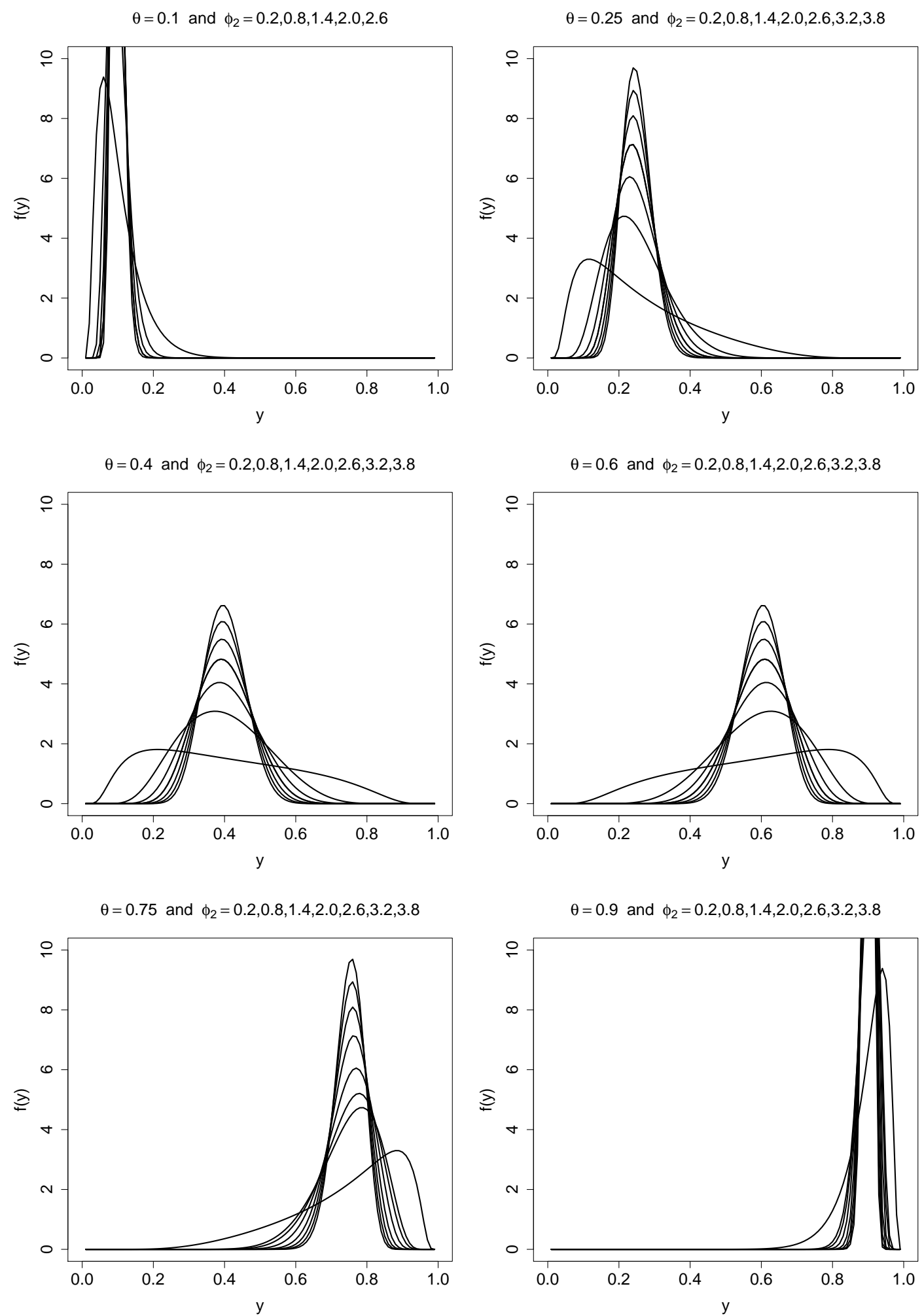

Figura B.2: Função de densidade de probabilidade para a distribuição limitada Simplex. 

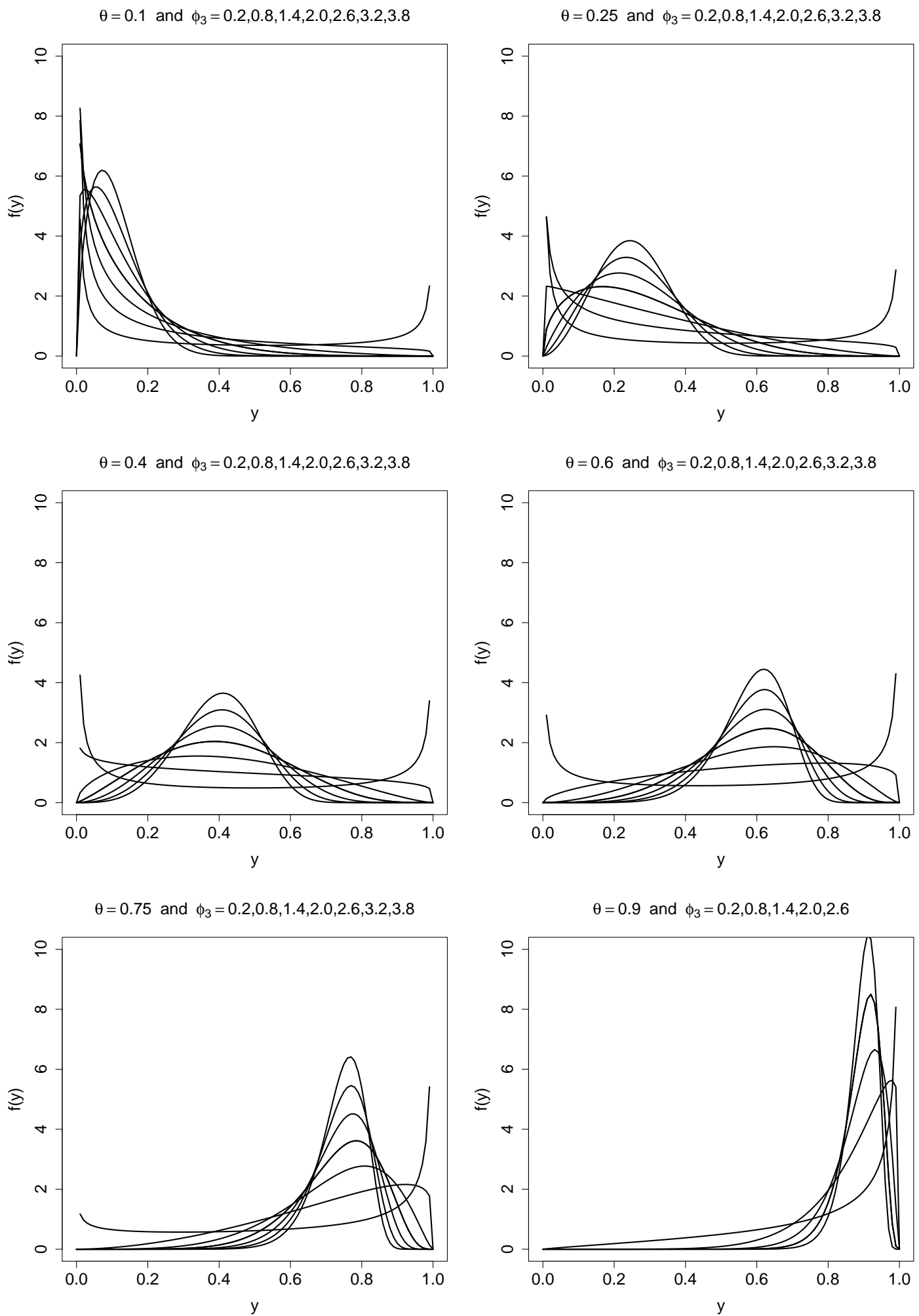

Figura B.3: Função de densidade de probabilidade para a distribuição limitada Kumaraswamy. 


\section{Apêndice C}

\section{O método INLA}

INLA, o acrónimo da aproximação integrada e anidada de Laplace, é uma abordagem de estimação aproximada na inferência Bayesiana para uma clase de modelos denominados como Modelos latentes gausianos (LGM). O LGM caracteriza a abstração de uma clase de modelos estadísticos Rue et al. $(2009,2017)$ os quais são definidos de forma hierárquica, onde um conjunto de $n$ observações $\boldsymbol{y}$ são definidos como sendo condicionalmente independentes dado o campo aleatório gausiano latente $\boldsymbol{\zeta}$ e os hiperparâmetros $\gamma_{1}$ como a seguir:

$$
\boldsymbol{y} \mid \boldsymbol{\zeta}, \gamma_{1} \sim \prod_{i=1}^{n} \pi\left(y_{i} \mid \zeta_{i}, \gamma_{1}\right)
$$

com

$$
\zeta \mid \gamma_{2} \sim \mathcal{N}\left(\mu\left(\gamma_{2}\right), Q^{-1}\left(\gamma_{2}\right)\right)
$$

onde o campo latente Gausiano, $\boldsymbol{\zeta}$, inclue tudos os termos aleatórios presentes no modelo e descreve a estrutura de dependência dos dados. Adicionalmente, os hiperparâmetros $\gamma=\left(\gamma_{1}, \gamma_{2}\right)$ regulam o campo latente Gausiano e ou a verosimilhança dados os dados. Assim, a distribuição a posteriori do modelo define-se como a seguir:

$$
\pi(\boldsymbol{\zeta}, \boldsymbol{\gamma} \mid \boldsymbol{y}) \propto \pi(\boldsymbol{\gamma}) \pi(\boldsymbol{\zeta} \mid \boldsymbol{\gamma}) \prod_{i=1}^{n} \pi\left(y_{i} \mid \zeta_{i}, \boldsymbol{\gamma}\right)
$$

e as marginais, definidas da seguinte forma,

$\pi\left(\zeta_{i} \mid \boldsymbol{y}\right)=\int \pi\left(\zeta_{i} \mid \boldsymbol{\gamma}, \boldsymbol{y}\right) \pi(\gamma \mid \boldsymbol{y}) d \boldsymbol{\gamma}$

$\pi\left(\gamma_{j} \mid \boldsymbol{y}\right)=\int \pi(\gamma \mid \boldsymbol{y}) d \gamma_{j}$,

são aproximadas no INLA como a seguir:

$$
\begin{gathered}
\tilde{\pi}\left(\zeta_{i} \mid \boldsymbol{y}\right)=\sum_{k} \tilde{\pi}\left(\zeta_{i} \mid \boldsymbol{\gamma}^{(k)}, \boldsymbol{y}\right) \tilde{\pi}\left(\boldsymbol{\gamma}^{(k)} \mid \boldsymbol{y}\right) \Delta \boldsymbol{\gamma}^{(k)} \\
\tilde{\pi}\left(\gamma_{j} \mid \boldsymbol{y}\right)=\int \tilde{\pi}(\gamma \mid \boldsymbol{y}) d \boldsymbol{\gamma}_{j},
\end{gathered}
$$

onde $\tilde{\pi}\left(\boldsymbol{\gamma}^{(k)} \mid \boldsymbol{y}\right)$ são quantidades da densidade calculada na exploração em rede na função $\tilde{\pi}(\boldsymbol{\gamma} \mid \boldsymbol{y})$ ?.

Com o intuito de identificar a solução das marginais para o campo latente na equação (C.4) é preciso achar pontos de avaliação adequados, estes pontos são o resultado da estratégia de exploração em rede. Tal exploração é um método para encontrar um conjunto de pontos de avaliação os quais 
abrangem a área onde a densidade de $\tilde{\pi}(\gamma \mid \boldsymbol{y})$ é maior. Nesse sentido, o trabalho computacional aumenta de forma exponencial de acordo com a dimensão de $\gamma$. Quando a dimensão de $\gamma$ é maior a estratégia denominada como integração CCD é utilizada para localizar os "pontos" com menor esforço computational.

As marginais a posteriori dos hiperparâmetros, $\tilde{\pi}\left(\gamma_{j} \mid \boldsymbol{y}\right)$ são calculados, de forma automática, através do algoritmo da integral numérica livre o qual é descrito no paper ? seção 3.2.2. 


\section{Apêndice D}

\section{Resultados adicionais no estudo de recuperação do capítulo 3}

Neste apêndice são apresentados resultados adicionais para o estudo de simulação de recuperação dos parâmetros. O estudo considera conjuntos de dados simulados do modelo SBDR-Ler e ajustado com os modelos SBDR-Ler, SBDR-Icar e também sem considerar o componente $\boldsymbol{z}$ (o modelo BR). 


\begin{tabular}{|c|c|c|c|c|c|c|c|c|}
\hline & Parâmetro & $\beta_{0}$ & $\beta_{1}$ & $\beta_{2}$ & $\beta_{3}$ & $\phi$ & $\tau$ & $\lambda$ \\
\hline \multicolumn{9}{|c|}{ Distribuição Beta } \\
\hline \multirow{5}{*}{ SBDR-Ler } & Original & -3 & 2 & 0.7 & 1.5 & 80 & 0.5 & 0.9 \\
\hline & Estimativa & -2.971 & 2.029 & 0.689 & 1.476 & 59.261 & 0.659 & 0.85 \\
\hline & $\mathrm{DP}$ & 0.423 & 0.556 & 0.052 & 0.124 & 18.704 & 0.132 & 0.096 \\
\hline & VA & 0.304 & 0.412 & 0.046 & 0.118 & 21.212 & 0.164 & 97 \\
\hline & Cobertura do $95 \%$ IC & 91 & 96 & 95 & 93 & 85 & 88 & \\
\hline \multirow[t]{4}{*}{ SBDR-Icar } & Estimativa & -2.951 & 2.015 & 0.685 & 1.466 & 53.254 & 0.665 & \\
\hline & $\mathrm{DP}$ & 0.154 & 0.541 & 0.055 & 0.141 & 30.088 & 0.241 & \\
\hline & VA & 0.304 & 0.421 & 0.047 & 0.12 & 27.065 & 0.173 & \\
\hline & Cobertura do $95 \%$ IC & 58 & 97 & 94 & 94 & 67 & 89 & \\
\hline \multirow[t]{4}{*}{$\mathrm{BR}$} & Estimativa & -2.41 & 1.672 & 0.564 & 1.138 & 7.534 & & \\
\hline & $\mathrm{DP}$ & 0.183 & 0.588 & 0.056 & 0.143 & 0.747 & & \\
\hline & VA & 0.604 & 0.614 & 0.137 & 0.362 & 72.466 & & \\
\hline & Cobertura do $95 \%$ IC & 27 & 86 & 32 & 31 & 0 & & \\
\hline \multicolumn{9}{|c|}{ Distribuição Kumaraswamy } \\
\hline & Original & -3 & 2 & 0.7 & 1.5 & 12 & 0.5 & 0.9 \\
\hline \multirow[t]{4}{*}{ SBDR-Ler } & Estimativa & -3.026 & 2.062 & 0.697 & 1.509 & 8.006 & 0.632 & 0.866 \\
\hline & $\mathrm{DP}$ & 0.436 & 0.541 & 0.049 & 0.118 & 1.504 & 0.1 & 0.088 \\
\hline & VA & 0.289 & 0.417 & 0.039 & 0.092 & 3.994 & 0.136 & 0.062 \\
\hline & Cobertura do $95 \%$ IC & 90 & 98 & 92 & 96 & 28 & 78 & 96 \\
\hline \multirow[t]{4}{*}{ SBDR-Icar } & Estimativa & -3.026 & 2.063 & 0.697 & 1.51 & 7.677 & 0.606 & \\
\hline & $\mathrm{DP}$ & 0.142 & 0.531 & 0.049 & 0.116 & 1.423 & 0.098 & \\
\hline & VA & 0.289 & 0.421 & 0.039 & 0.091 & 4.323 & 0.113 & \\
\hline & Cobertura do $95 \%$ IC & 58 & 97 & 90 & 96 & 16 & 84 & \\
\hline \multirow[t]{4}{*}{$\mathrm{BR}$} & Estimativa & -2.892 & 1.898 & 0.663 & 1.432 & 2.246 & & \\
\hline & $\mathrm{DP}$ & 0.237 & 0.691 & 0.064 & 0.198 & 0.128 & & \\
\hline & VA & 0.347 & 0.711 & 0.064 & 0.153 & 9.754 & & \\
\hline & Cobertura do $95 \%$ IC & 72 & 90 & 91 & 96 & 0 & & \\
\hline \multicolumn{9}{|c|}{ Distribuição Simplex } \\
\hline \multirow{5}{*}{ SBDR-Ler } & Original & -3 & 2 & 0.7 & 1.5 & 19 & 0.5 & 0.9 \\
\hline & Estimativa & -2.957 & 2.004 & 0.69 & 1.505 & 10.201 & 0.565 & 0.841 \\
\hline & $\mathrm{DP}$ & 0.372 & 0.51 & 0.046 & 0.102 & 8.977 & 0.075 & 0.088 \\
\hline & VA & 0.288 & 0.385 & 0.037 & 0.083 & 8.799 & 0.086 & 0.079 \\
\hline & Cobertura do $95 \%$ IC & 91 & 94 & 95 & 95 & 84 & 91 & 95 \\
\hline \multirow[t]{4}{*}{ SBDR-Icar } & Estimativa & -2.929 & 1.964 & 0.686 & 1.493 & 8.281 & 0.547 & \\
\hline & DP & 0.123 & 0.503 & 0.046 & 0.1 & 296622.8 & 0.061 & \\
\hline & VA & 0.311 & 0.406 & 0.04 & 0.094 & 10.719 & 0.089 & \\
\hline & Cobertura do $95 \%$ IC & 51 & 93 & 92 & 92 & 70 & 85 & \\
\hline \multirow[t]{4}{*}{$\mathrm{BR}$} & Estimativa & -2.625 & 1.713 & 0.603 & 1.383 & 0.125 & & \\
\hline & $\mathrm{DP}$ & 0.177 & 0.699 & 0.057 & 0.133 & 0.013 & & \\
\hline & VA & 0.46 & 0.843 & 0.107 & 0.192 & 18.875 & & \\
\hline & Cobertura do $95 \%$ IC & 42 & 85 & 57 & 72 & 0 & & \\
\hline
\end{tabular}

Tabela D.1: Estudo de recuperação dos parâmetros para o modelo simulado SBDR-Ler, considerando três distribuições limitadas e precisão espacial $\tau=0.5$ 


\begin{tabular}{|c|c|c|c|c|c|c|c|c|}
\hline & Parâmetro & $\beta_{0}$ & $\beta_{1}$ & $\beta_{2}$ & $\beta_{3}$ & $\phi$ & $\tau$ & $\lambda$ \\
\hline \multicolumn{9}{|c|}{ Distribuição Beta } \\
\hline \multirow{5}{*}{ SBDR-Ler } & Original & -3 & 2 & 0.7 & 1.5 & 80 & 2 & 0.9 \\
\hline & Estimativa & -2.983 & 2.018 & 0.691 & 1.484 & 63.781 & 3.049 & 0.867 \\
\hline & DP & 0.239 & 0.351 & 0.033 & 0.086 & 14.043 & 0.863 & 0.099 \\
\hline & VA & 0.169 & 0.297 & 0.028 & 0.067 & 16.719 & 1.078 & 0.062 \\
\hline & Cobertura do $95 \%$ IC & 96 & 97 & 94 & 96 & 77 & 81 & 95 \\
\hline \multirow[t]{4}{*}{ SBDR-Icar } & Estimativa & -2.973 & 2.013 & 0.688 & 1.479 & 58.627 & 3.435 & \\
\hline & $\mathrm{SD}$ & 0.106 & 0.345 & 0.033 & 0.086 & 12.147 & 1.147 & \\
\hline & VA & 0.168 & 0.299 & 0.029 & 0.068 & 21.588 & 1.46 & \\
\hline & Cobertura do $95 \%$ IC & 66 & 96 & 93 & 96 & 58 & 71 & \\
\hline \multirow[t]{4}{*}{$\mathrm{BR}$} & Estimativa & -2.808 & 1.927 & 0.653 & 1.371 & 22.789 & & \\
\hline & SD & 0.126 & 0.378 & 0.037 & 0.102 & 2.27 & & \\
\hline & VA & 0.223 & 0.408 & 0.053 & 0.135 & 57.211 & & \\
\hline & Cobertura do $95 \%$ IC & 58 & 88 & 71 & 78 & 0 & & \\
\hline \multicolumn{9}{|c|}{ Kumaraswamy distribution } \\
\hline & Original & -3 & 2 & 0.7 & 1.5 & 12 & 2 & 0.9 \\
\hline \multirow{4}{*}{ SBDR-Ler } & Estimativa & -3.011 & 2.034 & 0.699 & 1.508 & 9.669 & 2.753 & 0.848 \\
\hline & $\mathrm{DP}$ & 0.214 & 0.311 & 0.029 & 0.072 & 1.373 & 0.573 & 0.105 \\
\hline & VA & 0.158 & 0.249 & 0.022 & 0.053 & 2.331 & 0.765 & 0.073 \\
\hline & Cobertura do $95 \%$ IC & 90 & 96 & 96 & 95 & 65 & 74 & 97 \\
\hline \multirow[t]{4}{*}{ SBDR-Icar } & Estimativa & -3.012 & 2.035 & 0.699 & 1.51 & 9.296 & 2.758 & \\
\hline & $\mathrm{DP}$ & 0.087 & 0.305 & 0.028 & 0.071 & 1.286 & 0.625 & \\
\hline & VA & 0.158 & 0.252 & 0.022 & 0.052 & 2.704 & 0.774 & \\
\hline & Cobertura do $95 \%$ IC & 61 & 95 & 94 & 95 & 52 & 80 & \\
\hline \multirow[t]{4}{*}{$\mathrm{BR}$} & Estimativa & -3.032 & 2.077 & 0.714 & 1.536 & 4.311 & & \\
\hline & $\mathrm{DP}$ & 0.132 & 0.378 & 0.035 & 0.11 & 0.213 & & \\
\hline & VA & 0.183 & 0.427 & 0.035 & 0.085 & 7.689 & & \\
\hline & Cobertura do $95 \%$ IC & 74 & 84 & 91 & 97 & 0 & & \\
\hline \multicolumn{9}{|c|}{ Simplex distribution } \\
\hline \multirow{5}{*}{ SBDR-Ler } & Original & -3 & 2 & 0.7 & 1.5 & 19 & 2 & 0.9 \\
\hline & Estimativa & -3.001 & 2.016 & 0.698 & 1.5 & 14.063 & 2.29 & 0.847 \\
\hline & $\mathrm{DP}$ & 0.202 & 0.265 & 0.024 & 0.052 & 9.223 & 0.34 & 0.086 \\
\hline & VA & 0.151 & 0.189 & 0.019 & 0.044 & 5.06 & 0.354 & 0.08 \\
\hline & Cobertura do $95 \%$ IC & 88 & 95 & 94 & 92 & 93 & 95 & 92 \\
\hline \multirow[t]{4}{*}{ SBDR-Icar } & Estimativa & -3.001 & 2.015 & 0.698 & 1.501 & 12.125 & 2.168 & \\
\hline & $\mathrm{DP}$ & 0.063 & 0.26 & 0.023 & 0.051 & 7.873 & 0.322 & \\
\hline & VA & 0.151 & 0.19 & 0.019 & 0.043 & 6.969 & 0.29 & \\
\hline & Cobertura do $95 \%$ IC & 48 & 94 & 93 & 89 & 84 & 98 & \\
\hline \multirow[t]{4}{*}{$\mathrm{BR}$} & Estimativa & -2.913 & 1.97 & 0.679 & 1.468 & 0.501 & & \\
\hline & $\mathrm{DP}$ & 0.094 & 0.39 & 0.031 & 0.069 & 0.051 & & \\
\hline & VA & 0.177 & 0.355 & 0.051 & 0.082 & 18.499 & & \\
\hline & Cobertura do $95 \%$ IC & 58 & 94 & 62 & 82 & 0 & & \\
\hline
\end{tabular}

Tabela D.2: Estudo de recuperação dos parâmetros para o modelo simulado SBDR-Ler, considerando três distribuições limitadas e precisão espacial $\tau=2$ 
APÊNDICE D 


\section{Apêndice E}

\section{Resultados adicionais e o código usado na análise de dados reais do capítulo 3}

\section{E.1 Resultados adicionais}

$\mathrm{Na}$ presente seção apresenta-se os resultados adicionais para os dados da analise de dados reais do capítulo 3 onde os dados CL foram ajustados com os modelos SBDR-Icar sem o componente $\boldsymbol{z}$ (BR) e a versão sem o confundimento do modelo Leroux (SPOCK) (Prates et al., 2019) utilizando as distribuições limitadas Beta, Simplex e Kumaraswamy. Ademais, a avaliação do ajuste com os resíduos estandarizados e as densidades preditivas a posteriori (PPD) são apresentadas.

As figuras E.1, E.2 e E.3 aoresentam os residuos e a PPD para os diferentes modelos ajustados na aplicação de dados.

\section{E.2 código usado na análise de dados reais}

Esta seção apresenta o código no R das análisis do modelo sugerido de regressão espacial para respostas limitadas (SBDR).

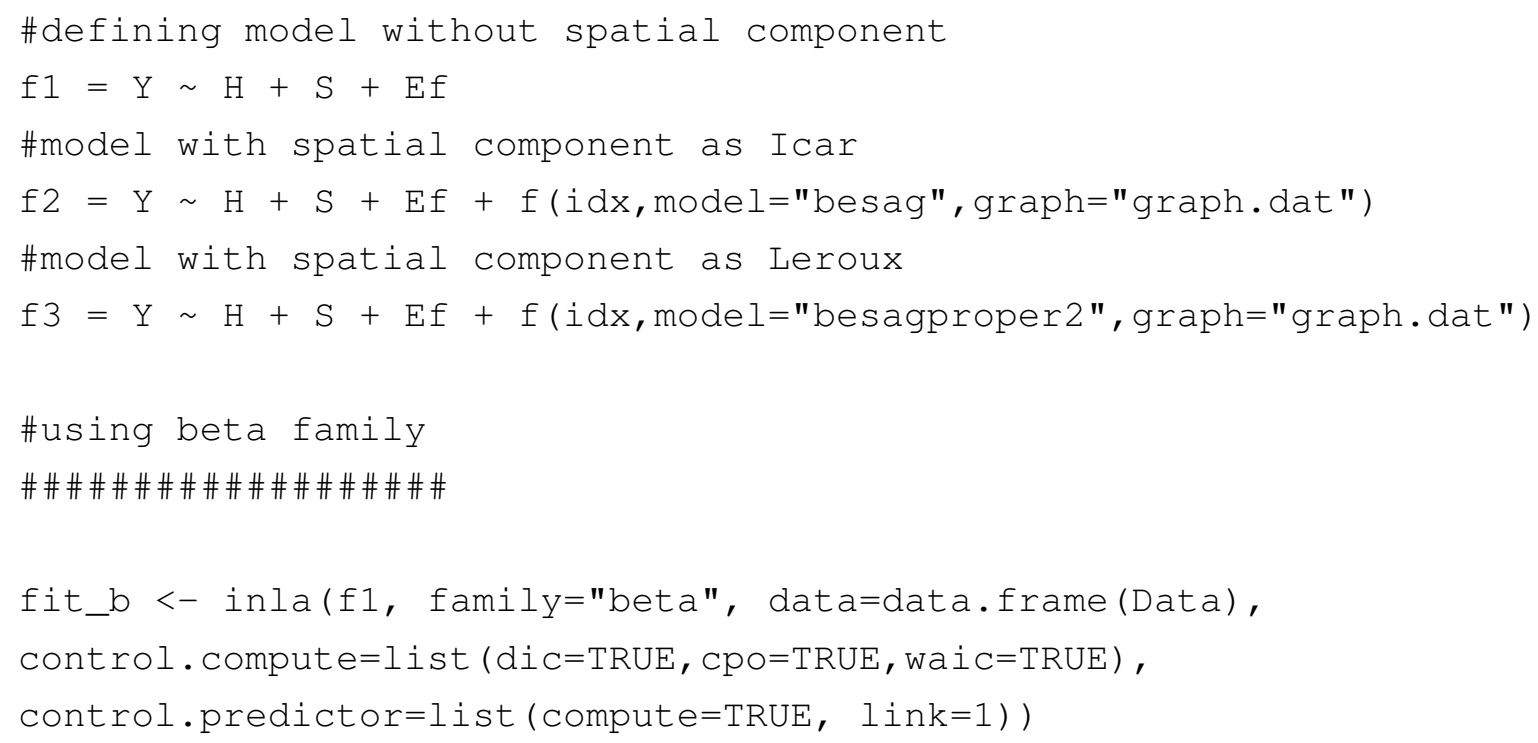




\begin{tabular}{|c|c|c|c|c|c|c|c|c|c|c|c|}
\hline \multicolumn{4}{|c|}{ Beta regression model } & \multicolumn{4}{|c|}{ SBDR-Icar Beta model } & \multicolumn{4}{|c|}{ SBDR-SPOCK Beta model } \\
\hline & mean & s.d. & $95 \% \mathrm{CI}$ & & mean & s.d. & $95 \% \mathrm{CI}$ & & mean & s.d. & $95 \% \mathrm{CI}$ \\
\hline$\beta_{0}$ & -3.64 & 0.19 & $(-4.01,-3.26)$ & $\beta_{0}$ & -3.52 & 0.16 & $(-3.85,-3.20)$ & $\beta_{0}$ & -3.70 & 0.21 & $(-4.11,-3.29)$ \\
\hline$\beta_{1}$ & 1.79 & 0.36 & $(1.08,2.50)$ & $\beta_{1}$ & 1.60 & 0.34 & $(0.92,2.28)$ & $\beta_{1}$ & 1.80 & 0.35 & $(1.10,2.48)$ \\
\hline$\beta_{2}$ & 1.27 & 0.27 & $(0.73,1.81)$ & $\beta_{2}$ & 0.82 & 0.25 & $(0.32,1.32)$ & $\beta_{2}$ & 1.25 & 0.27 & $(0.73,1.78)$ \\
\hline$\beta_{3}$ & 1.51 & 0.33 & $(0.86,2.17)$ & $\beta_{3}$ & 1.68 & 0.30 & $(1.10,2.27)$ & $\beta_{3}$ & 1.57 & 0.33 & $(0.93,2.20)$ \\
\hline \multirow[t]{3}{*}{$\phi$} & 24.79 & 2.49 & $(20.13,29.92)$ & $\phi$ & 79.65 & 16.60 & $(52.10,116.96)$ & $\phi$ & 41.50 & 7.52 & $(28.33,57.81)$ \\
\hline & & & & $\tau$ & 2.97 & 0.66 & $(1.86,4.44)$ & $\tau$ & 3.83 & 1.50 & $(1.78,7.57)$ \\
\hline & & & & & & & & $\lambda$ & 0.79 & 0.13 & $(0.48,0.96)$ \\
\hline \multicolumn{4}{|c|}{$\mathrm{DIC}=-476$} & \multicolumn{4}{|c|}{$\mathrm{DIC}=-627$} & \multicolumn{4}{|c|}{$\mathrm{DIC}=-529$} \\
\hline \multicolumn{4}{|c|}{$\mathrm{WAIC}=-476$} & \multicolumn{4}{|c|}{$\mathrm{WAIC}=-637$} & \multicolumn{4}{|c|}{$\mathrm{WAIC}=-529$} \\
\hline \multicolumn{4}{|c|}{$\mathrm{MAE}=0.0599$} & \multicolumn{4}{|c|}{$\mathrm{MAE}=0.0222$} & \multicolumn{4}{|c|}{$\mathrm{MAE}=0.0372$} \\
\hline \multicolumn{4}{|c|}{$\overline{\text { Simplex regression model }}$} & \multicolumn{4}{|c|}{ SBDR-Icar Simplex model } & \multicolumn{4}{|c|}{ SBDR-SPOCK Simplex model } \\
\hline & mean & s.d. & $95 \% \mathrm{CI}$ & & mean & s.d. & $95 \% \mathrm{CI}$ & & mean & s.d. & $95 \% \mathrm{CI}$ \\
\hline$\beta_{0}$ & -3.72 & 0.18 & $(-4.07,-3.35)$ & $\beta_{0}$ & -3.57 & 0.16 & $(-3.88,-3.25)$ & $\beta_{0}$ & -3.81 & 0.20 & $(-4.20,-3.41)$ \\
\hline$\beta_{1}$ & 2.15 & 0.49 & $(1.18,3.12)$ & $\beta_{1}$ & 1.72 & 0.38 & $(0.98,2.46)$ & $\beta_{1}$ & 1.87 & 0.41 & $(1.07,2.67)$ \\
\hline$\beta_{2}$ & 1.08 & 0.27 & $(0.55,1.60)$ & $\beta_{2}$ & 0.77 & 0.26 & $(0.25,1.29)$ & $\beta_{2}$ & 1.51 & 0.29 & $(0.95,2.07)$ \\
\hline$\beta_{3}$ & 1.67 & 0.32 & $(1.02,2.29)$ & $\beta_{3}$ & 1.69 & 0.30 & $(1.10,2.28)$ & $\beta_{3}$ & 1.40 & 0.34 & $(0.75,2.06)$ \\
\hline \multirow[t]{6}{*}{$\phi$} & 0.36 & 0.04 & $(0.29,0.43)$ & $\phi$ & 18.19 & 11.66 & $(4.52,48.47)$ & $\phi$ & 17.69 & 12.83 & $(3.75,51.30)$ \\
\hline & & & & $\tau$ & 1.26 & 0.14 & $(1.00,1.55)$ & $\tau$ & 1.27 & 0.36 & $(0.74,2.15)$ \\
\hline & & & & & & & & $\lambda$ & 0.38 & 0.14 & $(0.13,0.66)$ \\
\hline & \multicolumn{3}{|c|}{$\mathrm{DIC}=-442$} & \multicolumn{4}{|c|}{$\mathrm{DIC}=-986$} & \multicolumn{4}{|c|}{$\mathrm{DIC}=-982$} \\
\hline & \multicolumn{3}{|c|}{ WAIC $=-441$} & \multicolumn{4}{|c|}{ WAIC $=-1026$} & \multicolumn{4}{|c|}{ WAIC $=-1014$} \\
\hline & \multicolumn{3}{|c|}{$\mathrm{MAE}=0.0607$} & \multicolumn{4}{|c|}{$\mathrm{MAE}=0.0034$} & & & $\mathrm{MAE}=$ & 0028 \\
\hline$\overline{\mathrm{Ku}}$ & maraswe & my re & ression model & & $\overline{\text { DR-Ical }}$ & Kuma & aswamy model & & $\overline{\mathrm{DR}-\mathrm{SPC}}$ & $\overline{\mathrm{CK} \mathrm{Ku}}$ & "iraswamy model \\
\hline & mean & s.d. & $95 \%$ CI & & mean & s.d. & $95 \% \mathrm{CI}$ & & mean & s.d. & $95 \% \mathrm{CI}$ \\
\hline$\beta_{0}$ & -3.84 & 0.21 & $(-4.27,-3.43)$ & $\beta_{0}$ & -3.53 & 0.16 & $(-3.84,-3.21)$ & $\beta_{0}$ & -3.73 & 0.21 & $(-4.15,-3.32)$ \\
\hline$\beta_{1}$ & 1.80 & 0.38 & $(1.06,2.54)$ & $\beta_{1}$ & 1.64 & 0.34 & $(0.97,2.31)$ & $\beta_{1}$ & 1.80 & 0.36 & $(1.09,2.51)$ \\
\hline$\beta_{2}$ & 1.32 & 0.30 & $(0.74,1.91)$ & $\beta_{2}$ & 0.74 & 0.25 & $(0.25,1.23)$ & $\beta_{2}$ & 1.35 & 0.27 & $(0.81,1.88)$ \\
\hline$\beta_{3}$ & 1.71 & 0.37 & $(0.97,2.44)$ & $\beta_{3}$ & 1.73 & 0.29 & $(1.15,2.30)$ & $\beta_{3}$ & 1.52 & 0.33 & $(0.87,2.16)$ \\
\hline$\phi$ & 4.22 & 0.21 & $(3.82,4.63)$ & $\phi$ & 10.92 & 1.64 & $(8.01,14.47)$ & $\phi$ & 7.02 & 1.08 & $(5.08,9.30)$ \\
\hline & & & & $\tau$ & 2.07 & 0.37 & $(1.45,2.88)$ & $\tau$ & 2.03 & 0.57 & $(1.18,3.40)$ \\
\hline & & & & & & & & $\lambda$ & 0.70 & 0.15 & $(0.37,0.93)$ \\
\hline & & $\mathrm{IC}=$ & 469 & & & $\mathrm{DIC}=$ & 720 & & & $\mathrm{DIC}=$ & 572 \\
\hline & & $\mathrm{AIC}=$ & -469 & & & $\mathrm{IAIC}=$ & -725 & & & WAIC & -573 \\
\hline & M & $\mathrm{E}=$ & .0595 & & & $\mathrm{AE}=$ & .0133 & & & MAE $=$ & 0256 \\
\hline
\end{tabular}

Tabela E.1: Média a posteriori (Média), desvío padrao (DP) e o intervalo de credibilidade (IC) de 95\% para os parâmetros estimados dos dados de CL considerando diferentes modelos espaciasi para variáveis de respostas limitadas, sob os modelos SBDR-Icar, BR e SPOCK. 

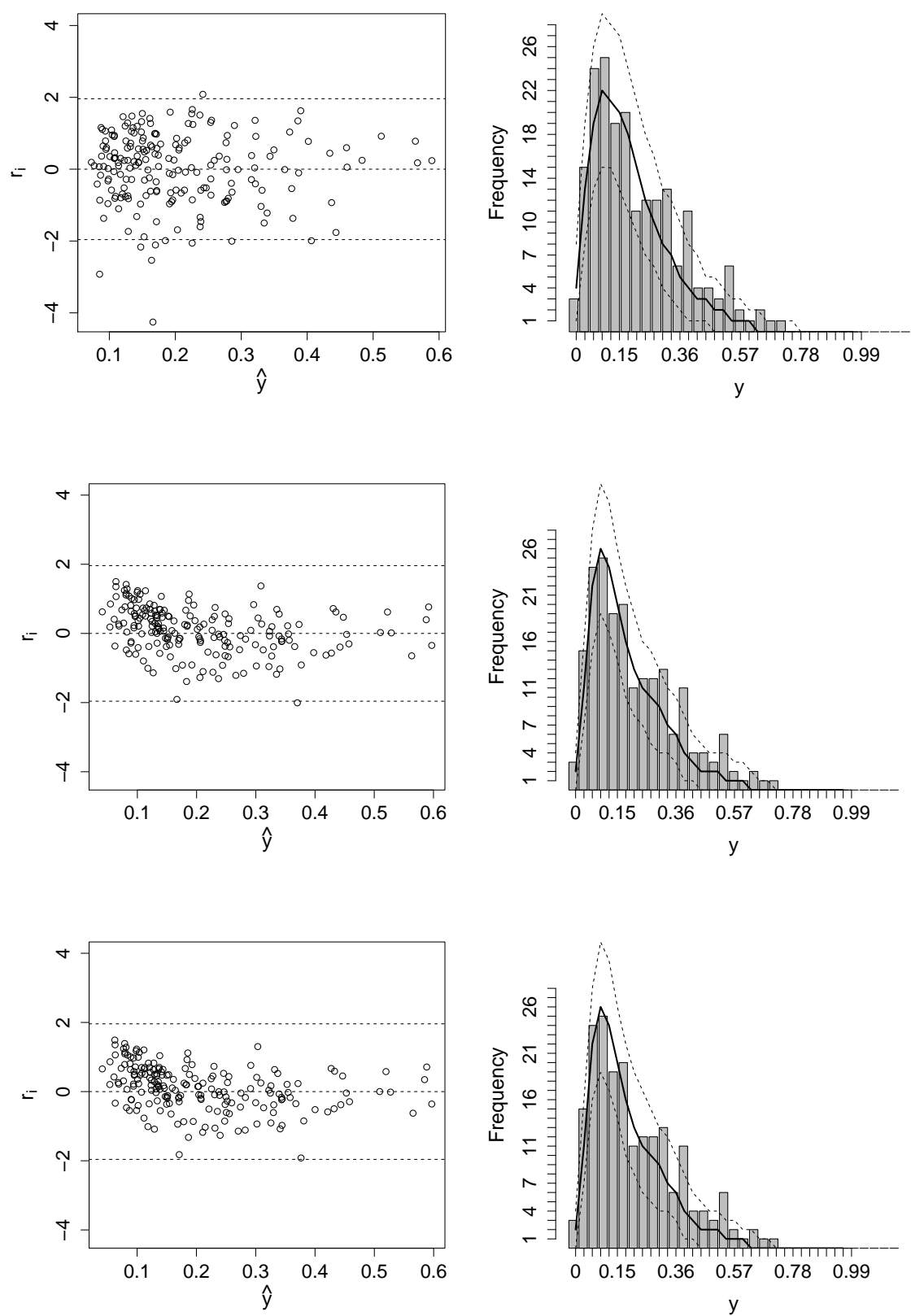

Figura E.1: Ajuste dos modelos Beta: Resíduos padrão $\left(r_{i}\right)$ vs respostas ajustads ( $\hat{y}$ ) (esquerda) e banda de confiança da PPD com o histograma observado (dereita). BR (parte superior), SBDR-Icar (no médio) and SBDR-Ler (parte inferior). 

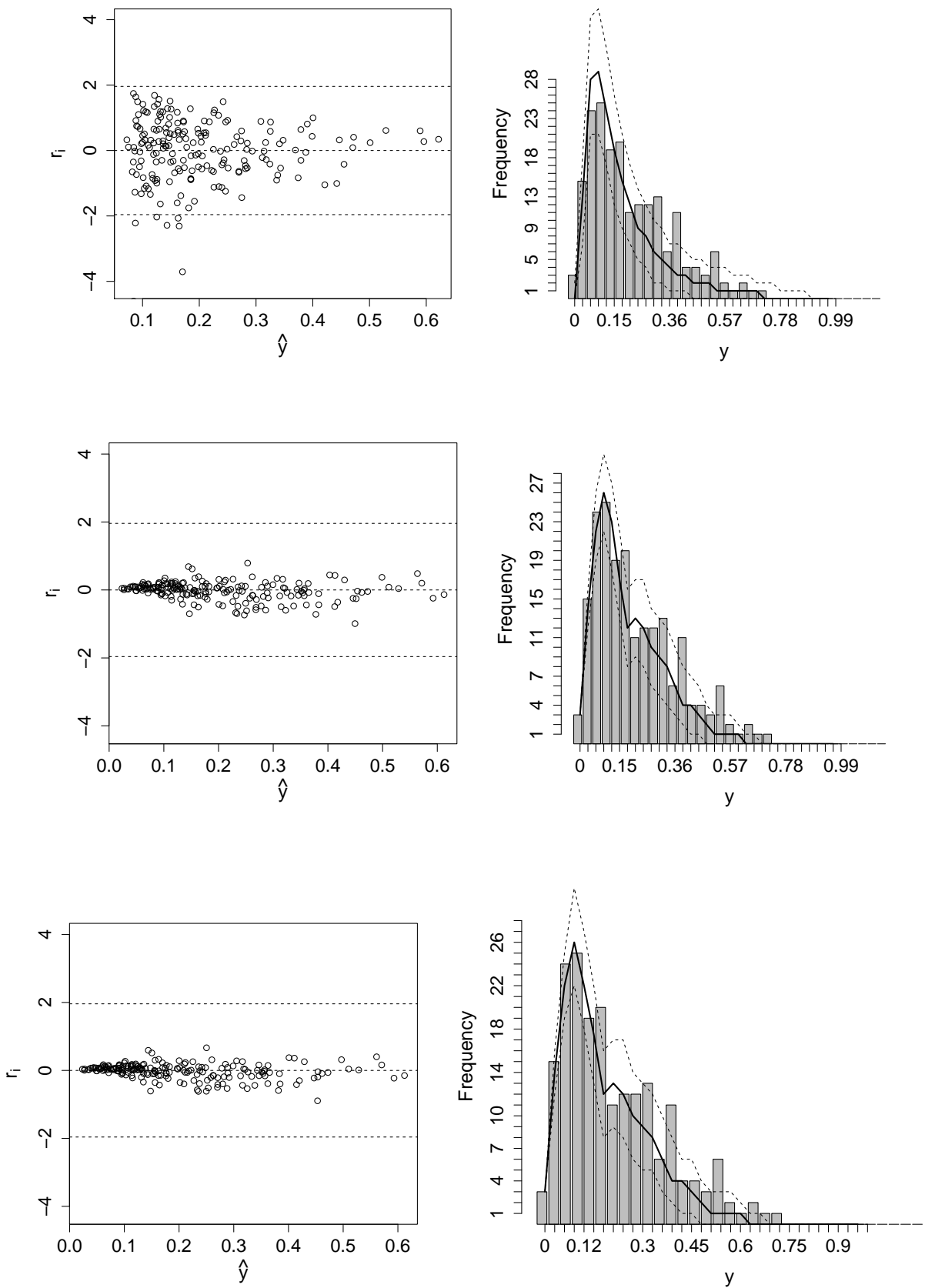

Figura E.2: Ajuste dos modelos Simplex: Resíduos padrão $\left(r_{i}\right)$ vs respostas ajustads $(\hat{y})$ (esquerda) e banda de confiança da PPD com o histograma observado (dereita). BR (parte superior), SBDR-Icar (no médio) and SBDR-Ler (parte inferior). 

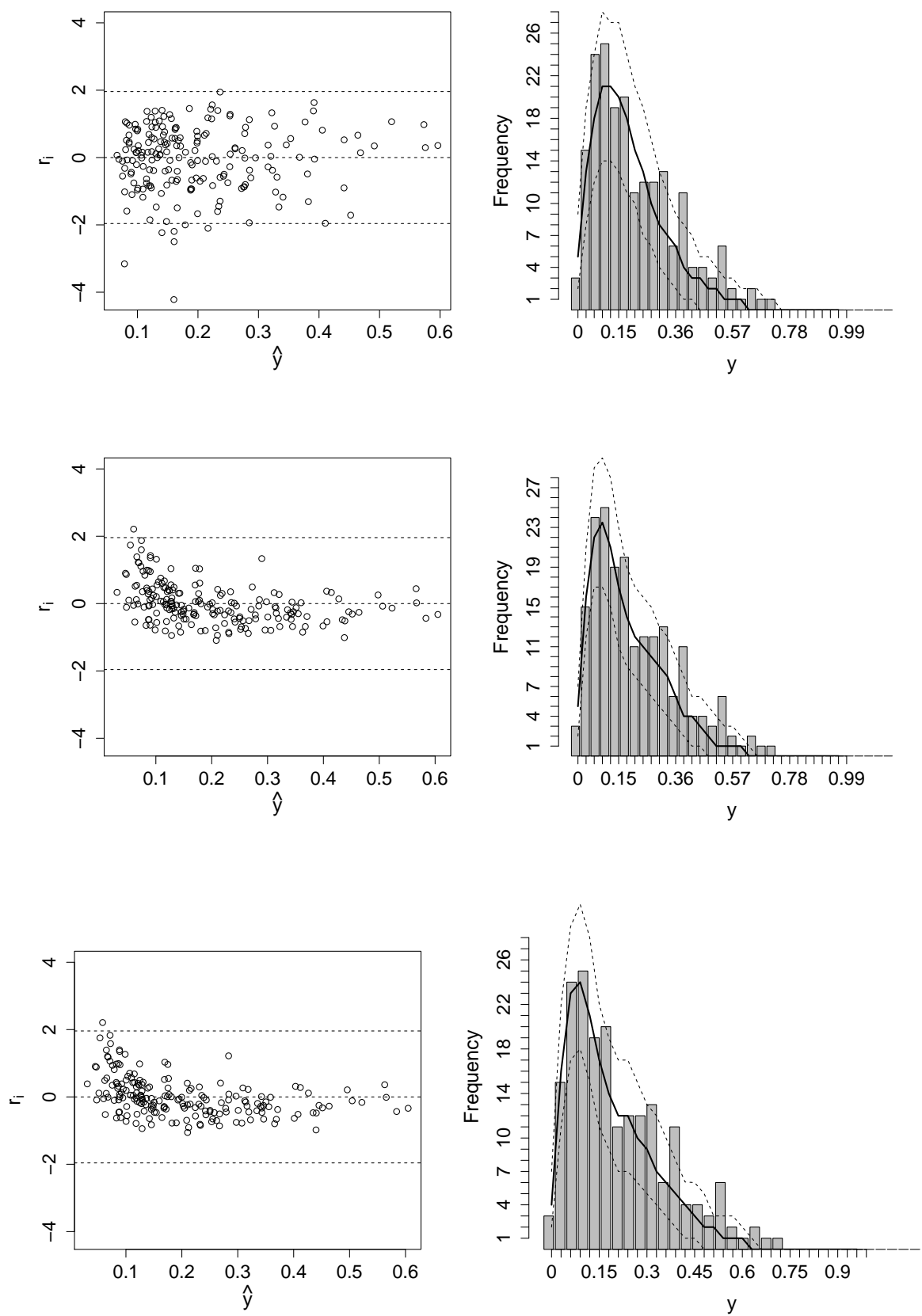

Figura E.3: Ajuste dos modelos Kumaraswamy: Resíduos padrão $\left(r_{i}\right)$ vs respostas ajustads ( $\left.\hat{y}\right)$ (esquerda) e banda de confiança da PPD com o histograma observado (dereita). BR (parte superior), SBDR-Icar (no médio) and SBDR-Ler (parte inferior). 


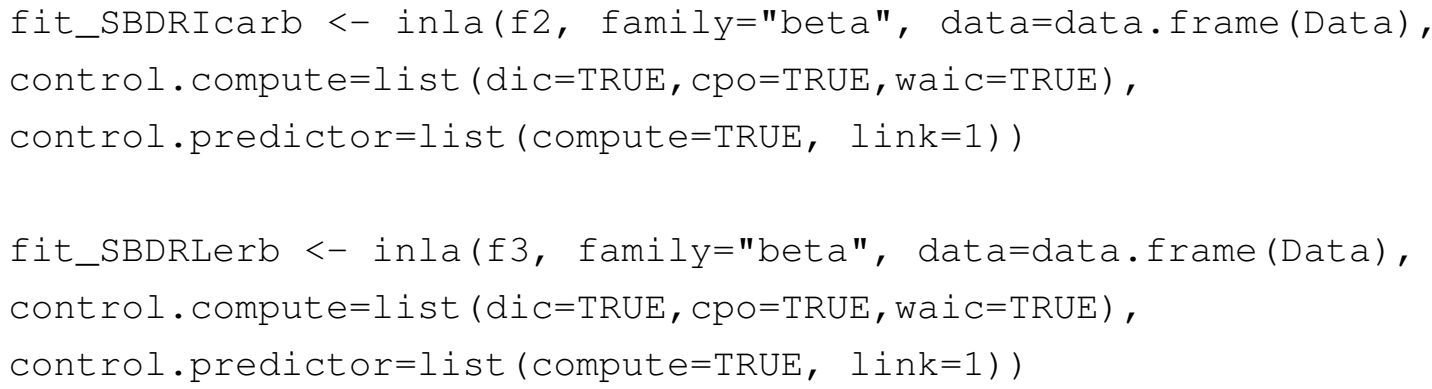




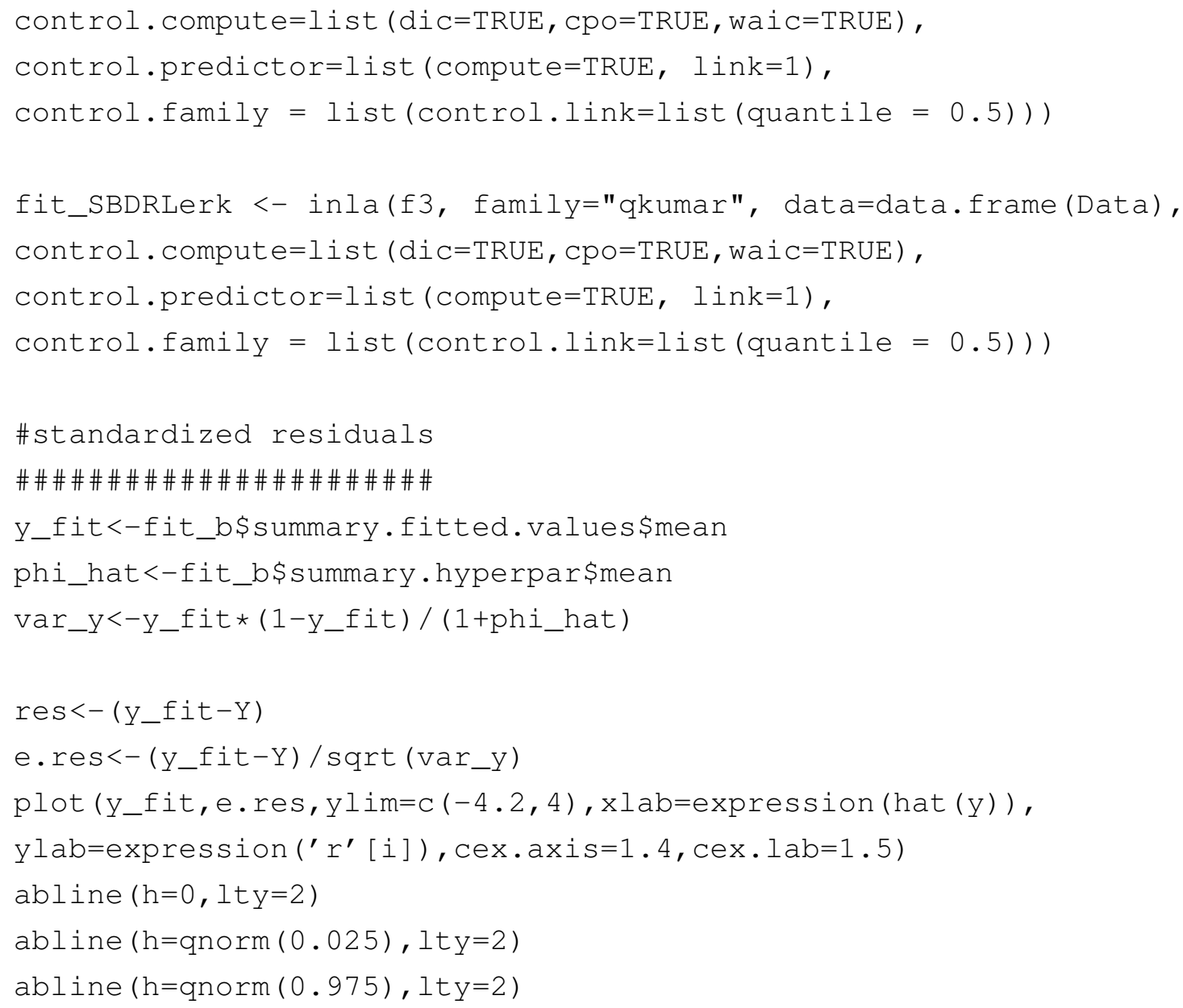


APÊNDICE E 


\section{Apêndice F}

\section{Códigos BUGS dos modelos HBRT-beta e HBRT-simplex}

Neste apêndice apresentamos os códigos BUGS para os modelos hierárquicos conjuntos do tempo de resposta limitado e a precisão (HBRT) sugeridos no capítulo 4.

\section{F.1 Código BUGS do modelo HBR-beta}

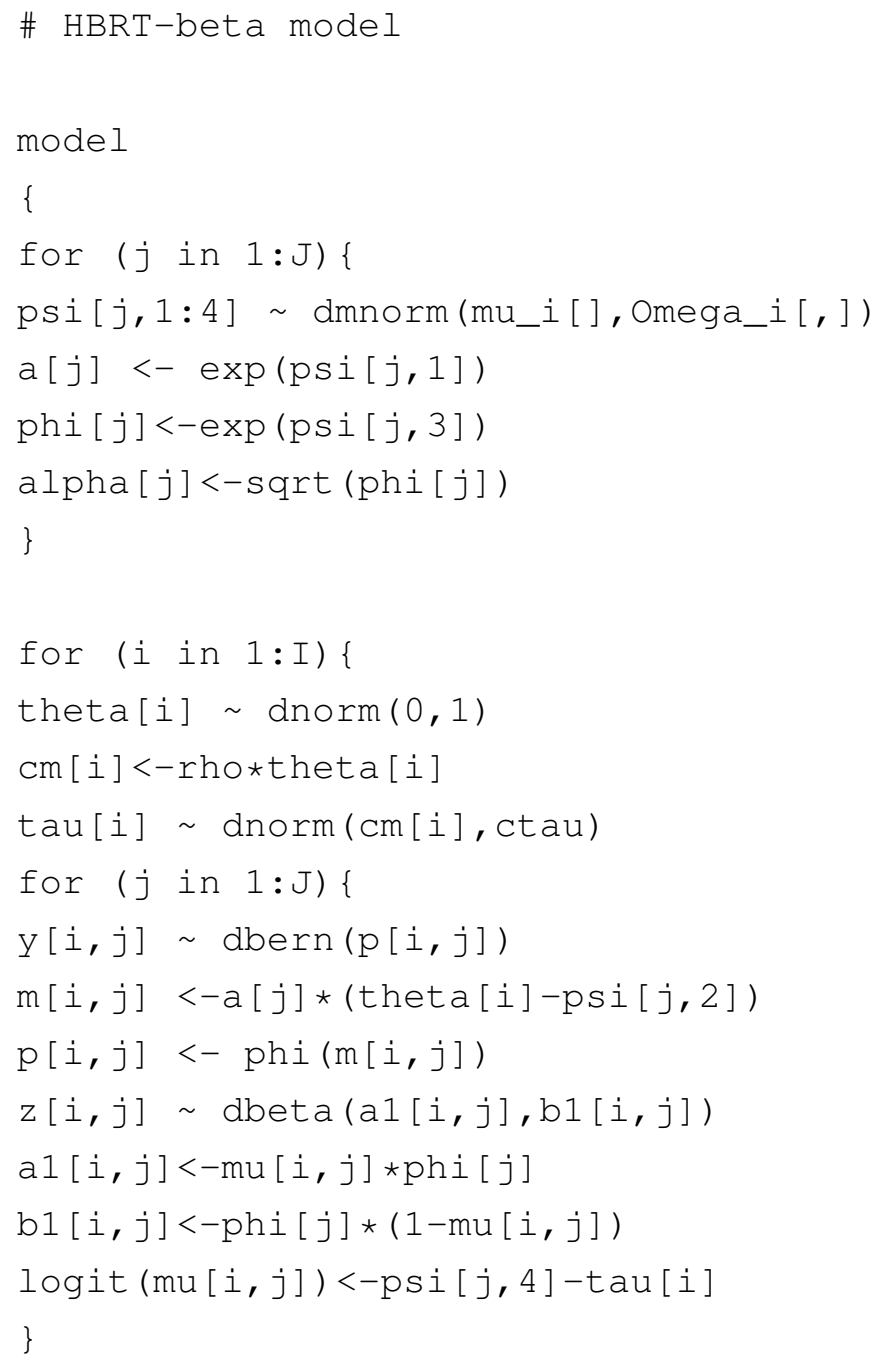


\}

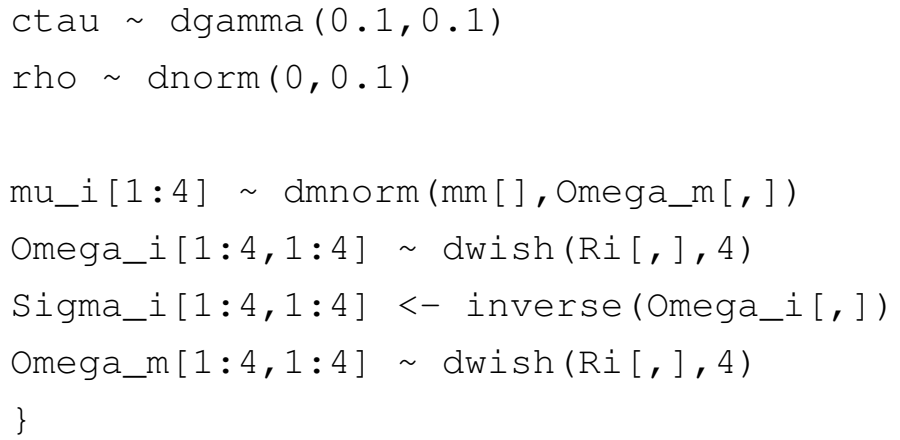

\section{F.2 Código BUGS do modelo HBRT-simplex}

O WinBUGS não contém a distribuição Simplex na lista de distribuições disponíveis para modelamento dos dados, nesse sentido implementamos o método "zero poisson" com o intuito de simular da distribuição Simplex, detalhes do método foram obtidos do manual do WinBUGS Spiegelhalter et al. (2003b).

\# Hierarchical bounded response time model
\# HBRT-simplex

model

\{

for $(j$ in $1: J)\{$

$\operatorname{psi}[j, 1: 4] \sim \operatorname{dmnorm}\left(m u \_i[], O m e g a \_i[],\right)$

a $[j]<-\exp (\operatorname{psi}[j, 1])$

$\operatorname{phi}[j]<-\exp (\operatorname{psi}[j, 3])$

alpha [j]<-sqrt (phi [j])

\}

for (i in 1:I) \{

theta $[i] \sim \operatorname{dnorm}(0,1)$

cm [i]<-rho*theta [i]

tau[i] $\operatorname{dnorm}(\mathrm{cm}[i], \mathrm{ctau})$

for $(j$ in $1: J)\{$

$\mathrm{y}[i, j] \sim \operatorname{dbern}(\mathrm{p}[i, j])$

$m[i, j]<-a[j] *($ theta $[i]-p s i[j, 2])$

$\mathrm{p}[i, j]<-\operatorname{phi}(\mathrm{m}[i, j])$

zeros $[i, j]<-0$

zeros $[i, j] \sim \operatorname{dpois}(1 i k[i, j])$

$\operatorname{lik}[i, j]<-\log (1000 \star 3.141593)-.5 \star \log (\operatorname{phi}[j])+1.5 \star \log (z[i, j] \star(1-z[i, j]))+$ 


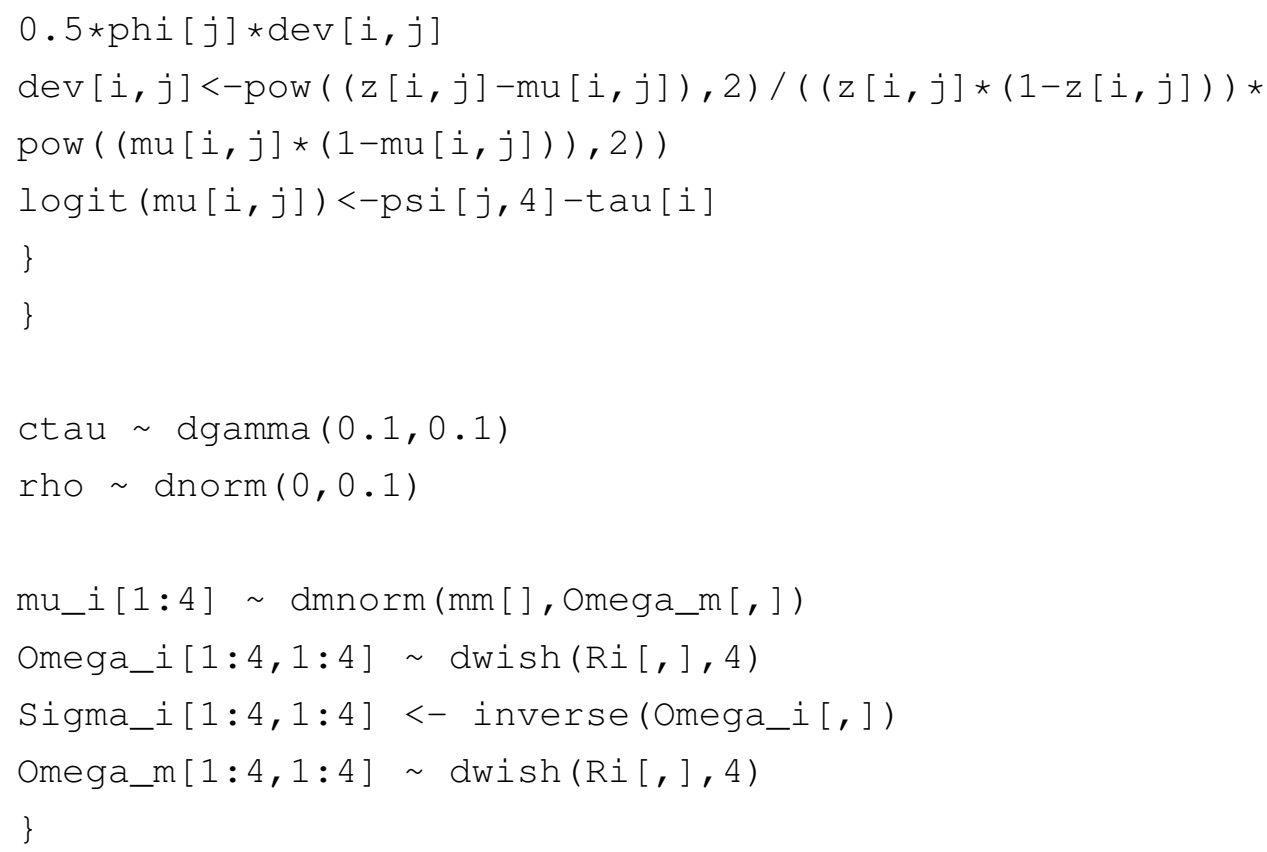


APÊNDICE F 


\section{Referências Bibliográficas}

Albert(1992) J. H. Albert. Bayesian estimation of normal ogive item response curves using gibbs sampling. Journal of Educational Statistics, 17 (3):251-269. Citado na pág. 8

Azevedo(2003) Caio Lucidius Naberezny Azevedo. Métodos de estimação na teoria de resposta ao item. Dissertação de Mestrado, Universidade de São Paulo. Citado na pág. 7

Azevedo(2008) Caio Lucidius Naberezny Azevedo. Modelos longitudinais de grupos múltiplos multiniveis na teoria da resposta ao item: métodos de estimação e seleção estrutural sob uma perspectiva bayesiana. Tese de Doutorado, Universidade de São Paulo. Citado na pág. 8

Barndorff-Nielsen e JØrgensen(1991) Ole E. Barndorff-Nielsen e Bent JØrgensen. Some parametric models on the simplex. Journal of Multivariate Analysis, 39(1):106 - 116. ISSN 0047-259X. doi: https://doi.org/10.1016/0047-259X(91)90008-P. Citado na pág. 38, 39

Bayes et al.(2017) C. L. Bayes, J. L. Bazán e M. de Castro. A quantile parametric mixed regression model for bounded response variables. Statistics and Its Interface, 10:483-493. Citado na pág. 17, 18

Besag e Kooperberg(1995) Julian Besag e Charles Kooperberg. On conditional and intrinsic autoregressions. Biometrika, 82(4):733-746. Citado na pág. 18

Besag et al.(1991) Julian Besag, Jeremy York e Annie Mollié. Bayesian image restoration, with two applications in spatial statistics. Annals of the Institute of Statistical Mathematics, 43(1). URL https://doi.org/10.1007/BF00116466. Citado na pág. 18, 24

Betancourt(2017) Michael Betancourt. A conceptual introduction to hamiltonian monte carlo. arXiv preprint arXiv:1701.02434. Citado na pág. 57

Betancourt e Girolami(2013) MJ Betancourt e Mark Girolami. Hamiltonian monte carlo for hierarchical models. arXiv preprint arXiv:1312.0906. Citado na pág. 6

Blangiardo e Cameletti(2015) Marta Blangiardo e Michela Cameletti. Spatial and Spatioâtemporal Bayesian Models with $R$ INLA. John Wiley and Sons. Citado na pág. 19, 20, 27, 28

Bonat et al.(2013) Wagner Hugo Bonat, Paulo Justiniano Riveiro Jr e Walmes Marques Zeviani. Regression models with responses on the unit interval: specification, estimation and comparison. Rev. Bras. Biom, 20(1):1-10. Citado na pág. 17

Bonat et al.(2014) Wagner Hugo Bonat, Paulo Justiniano Ribeiro Jr et al. Bayesian analysis for a class of beta mixed models. arXiv preprint arXiv:1401.295\%. Citado na pág. 27

Bradlow et al.(1999) E.T. Bradlow, H. Wainer e X. Wang. A bayesian random effects model for testlets. Psychometrika, 64 (2):153-168. Citado na pág. 2, 5, 7, 9

Carpenter et al.(2017) Bob Carpenter, Andrew Gelman, Matthew Hoffman, Daniel Lee, Ben Goodrich, Michael Betancourt, Marcus Brubaker, Jiqiang Guo, Peter Li e Allen Riddell. Stan: A probabilistic programming language. Journal of statistical software, 76(1):1-32. doi: 10.18637/ jss.v076.i01. URL https://www.jstatsoft.org/v076/i01. Citado na pág. 11, 36, 57 
Cepeda-Cuervo e Núñez-Antón(2013) E. Cepeda-Cuervo e V. Núñez-Antón. Spatial double generalized beta regression models. Extensions and application to study quality of education in Colombia. Journal of Educational and Behavioral Statistics, 38:604-628. Citado na pág. 17, 18, 21

da Paz et al.(2018) Rosineide F da Paz, Narayanaswamy Balakrishnan e Jorge Luis Bazán. L-logistic regression models: Prior sensitivity analysis, robustness to outliers and applications. Brazilian Journal of Probability and Statistics. Citado na pág. 17

Duane et al.(1987) Simon Duane, A.D. Kennedy, Brian J. Pendleton e Duncan Roweth. Hybrid monte carlo. Physics Letters B, 195(2):216 - 222. ISSN 0370-2693. doi: http://dx. doi.org/10.1016/0370-2693(87)91197-X. URL http://www.sciencedirect.com/science/article/pii/ 037026938791197X. Citado na pág. 6

Ferrari e Cribari-Neto(2004) S. L. P. Ferrari e F. Cribari-Neto. Beta regression for modelling rates and proportions. Journal of Applied Statistics, 31:799-815. Citado na pág. 17, 38

Figueroa-Zuñiga et al.(2013) J. I. Figueroa-Zuñiga, R. B. Arellano-Valle e S. L. P. Ferrari. Mixed beta regression: A bayesian perspective. Computational Statistics and Data Analysis, 61:137-147. Citado na pág. 17

Flegal et al.(2017) James M. Flegal, John Hughes, Dootika Vats e Ning Dai. mcmcse: Monte Carlo Standard Errors for MCMC. Riverside, CA, Denver, CO, Coventry, UK, and Minneapolis, MN, 2017. R package version 1.3-2. Citado na pág. 12

Flores Ari(2012) Sandra Flores Ari. Modelos testlet logisticos y logisticos de exponente positivo para pruebas de compresion de textos. Dissertação de Mestrado, Pontificia Universidad Catolica del Peru. Tesis para optar el grado de Magister en Estadistica - PUCP. Citado na pág. 5

Fox et al.(2007) Gerardus JA Fox, RH Klein Entink e Willem J van der Linden. Modeling of responses and response times with the package cirt. Journal of statistical software, 20(7):1-14. Citado na pág. 1, 37, 43

Gelman et al.(1995) Andrew Gelman, Jhohn B. Carlin, Hal S. Stern e Donald B. Rubin. Bayesian Data Analysis. Chapman \& Hall. Citado na pág. 8, 12, 43

Gelman et al.(2014) Andrew Gelman, Jessica Hwang e Aki Vehtari. Understanding predictive information criteria for bayesian models. Statistics and Computing, 24(6):997-1016. Citado na pág. 28

Gilks et al.(1995) Walter R Gilks, Sylvia Richardson e David Spiegelhalter. Markov chain Monte Carlo in practice. Chapman and Hall/CRC. Citado na pág. 10

Goudie et al.(in press) Robert JB Goudie, Rebecca M Turner, Daniela De Angelis e Andrew Thomas. Multibugs: Massively parallel mcmc for bayesian hierarchical models. Journal of Statistical Software. arXiv:1704.03216. Citado na pág. 36

Grant et al.(2016) Robert L Grant, Daniel C Furr, Bob Carpenter e Andrew Gelman. Fitting bayesian item response models in stata and stan. arXiv preprint arXiv:1601.03443. Citado na pág. 12

Hanks et al.(2015) Ephraim M Hanks, Erin M Schliep, Mevin B Hooten e Jennifer A Hoeting. Restricted spatial regression in practice: geostatistical models, confounding, and robustness under model misspecification. Environmetrics, 26(4):243-254. Citado na pág. 35

Hodges e Reich(2010) James S Hodges e Brian J Reich. Adding spatially-correlated errors can mess up the fixed effect you love. The American Statistician, 64(4):325-334. Citado na pág. 35 
Hoffman e Gelman(2014) Matthew D Hoffman e Andrew Gelman. The no-u-turn sampler: adaptively setting path lengths in hamiltonian monte carlo. Journal of Machine Learning Research, 15(1):1593-1623. Citado na pág. 11, 58

Huey(1898) Edmund B Huey. Preliminary experiments in the psysiology and psychology of reading. American Journal of Psychology, 9:575-586. Citado na pág. 1

Hughes e Haran(2013) John Hughes e Murali Haran. Dimension reduction and alleviation of confounding for spatial generalized linear mixed models. Journal of the Royal Statistical Society: Series B (Statistical Methodology), 75(1):139-159. Citado na pág. 35

$\operatorname{Im}(\mathbf{2 0 1 5})$ Suk Keun Im. The Hierarchical Testlet Response Time Model: Bayesian analysis of a testlet model for item responses and response times. Tese de Doutorado, University of Kansas. Citado na pág. 1, 37, 43

Jang(2009) Eunice Eunhee Jang. Cognitive diagnostic assessment of 12 reading comprehension ability: Validity arguments for fusion model application to languedge assessment. Language Testing, 26(1):031-73. Citado na pág. 1

Johnson et al.(1995) Norman Lloyd Johnson, Samuel Kotz e Narayanaswamy Balakrishnan. Continuous univariate distributions. Citado na pág. 38, 39

JØrgensen(1997) Bent JØrgensen. The theory of dispersion models. Chapman and Hall, London. Citado na pág. 22, 38, 39

Kennedy(1930) Margaret Kennedy. Speed as a personality trait. The Journal of Social Psychology, 1(2):286-299. doi: 10.1080/00224545.1930.9918819. URL https://doi.org/10.1080/00224545.1930. 9918819. Citado na pág. 37

Kieschnick e McCullough(2003) R. Kieschnick e B. D. McCullough. Regression analysis of variates observed on $(0,1)$ : percentages, proportions and fractions. Statistical Modelling, 3:193213. Citado na pág. 17

Klein Entink(2009) Rinke Hermen Klein Entink. Statistical models for responses and response times. Tese de Doutorado, University of Twente. Citado na pág. 1, 37, 43

Kumaraswamy(1976) P Kumaraswamy. Sinepower probability density function. Journal of Hydrology, 31(1-2):181-184. Citado na pág. 39

Lemonte e Bazán(2016) Artur J Lemonte e Jorge L Bazán. New class of johnson distributions and its associated regression model for rates and proportions. Biometrical Journal, 58(4):727-746. Citado na pág. 17

Leroux et al.(2000) Brian G Leroux, Xingye Lei e Norman Breslow. Estimation of disease rates in small areas: a new mixed model for spatial dependence. Em Statistical models in epidemiology, the environment, and clinical trials, páginas 179-191. Springer. Citado na pág. 2, 18, 26, 32

Levy(2009) Roy Levy. The rise of markov chain monte carlo estimation for psychometric modeling. Journal of Probability and Statistics, 2009. Citado na pág. 6

López(2013) Freddy Omar López. A bayesian approach to parameter estimation in simplex regression model: A comparison with beta regression. Revista Colombiana de Estad Astica, 36(1): 1-21. Citado na pág. 18

Lord e Novick(1968) F.M. Lord e M. Novick. Statistical Theories of Mental Tests Scores. AddisonWesley. Citado na pág. 5 
Lunn et al.(2012) David Lunn, Chris Jackson, Nicky Best, David Spiegelhalter e Andrew Thomas. The BUGS book: A practical introduction to Bayesian analysis. Chapman and Hall/CRC. Citado na pág. 11

Lunn et al.(2000) David J Lunn, Andrew Thomas, Nicky Best e David Spiegelhalter. Winbugs-a bayesian modelling framework: concepts, structure, and extensibility. Statistics and computing, 10(4):325-337. Citado na pág. 36, 44

McKay(2010) Curtis S. McKay. Bugs code for item response theory. Journal of Statistical Software, 36. Citado na pág. 6

MINEDU(2016) MINEDU. Marco de fundamentación de las pruebas de la Evaluación Censal de Estudiantes. Ministerio de Educación del Perú, Lima. Citado na pág. 19, 32

Natesan et al.(2016) Prathiba Natesan, Ratna Nandakumar, Tom Minka e Jonathan D Rubright. Bayesian prior choice in irt estimation using mcmc and variational bayes. Frontiers in psychology, 7:1422. Citado na pág. 9

OECD(2017) OECD. PISA 2015 Technical Report. Relatório técnico, OECD. Citado na pág. 47

Paek et al.(2009) Insu Paek, Haniza Yon, Mark Wilson e Taehoon Kang. Random parameter structure and the testlet model: Extension of the rasch testlet model. Journal of applied measurement, 10(4):394-407. Citado na pág. 5

Perez(2014) Elena Jimenez Perez. Comprensión lectora vs competencia lectora:. Investigaciones sobre lectura, (1):65-74. Citado na pág. 1

Plummer(2012) Martyn Plummer. Jags version 3.3. 0 user manual. International Agency for Research on Cancer, Lyon, France. Citado na pág. 36

PNUD(2013) PNUD. Informe sobre desarrollo humano Perú 2013: Cambio climático y territorio: Desafíos y respuestas para un futuro sostenible. Programa de las Naciones Unidas para el Desarrollo. Lima. Citado na pág. 19, 32

Prates et al.(2019) Marcos Oliveira Prates, Renato Martins Assunção e Erica Castilho Rodrigues. Alleviating spatial confounding for areal data problems by displacing the geographical centroids. Bayesian Analysis, 14(2):623-647. Citado na pág. 35, 71

Qiu et al.(2008) Zhenguo Qiu, PETER X-K SONG e Ming Tan. Simplex mixed-effects models for longitudinal proportional data. Scandinavian Journal of Statistics, 35(4):577-596. Citado na pág. 17

Quintero(2017) Freddy Omar López Quintero. Sensitivity analysis for variance parameters in bayesian simplex mixed models for proportional data. Communications in Statistics-Simulation and Computation, 46(7):5212-5228. Citado na pág. 40

Reich et al.(2006) Brian J Reich, James S Hodges e Vesna Zadnik. Effects of residual smoothing on the posterior of the fixed effects in disease-mapping models. Biometrics, 62(4):1197-1206. Citado na pág. 35

Rosenbaum(1988) Paul R. Rosenbaum. Items bundles. Psychometrika, 53(3):349-359. ISSN 1860-0980. doi: 10.1007/BF02294217. URL http://dx.doi.org/10.1007/BF02294217. Citado na pág. 5

Rue e Held(2005) Havard Rue e Leonhard Held. Gaussian Markov Random Field: Theory and Applications. Chapman and Hall / CRC. Citado na pág. 2 
Rue et al.(2009) Håvard Rue, Sara Martino e Nicolas Chopin. Approximate bayesian inference for latent gaussian models by using integrated nested laplace approximations. Journal of the royal statistical society: Series b (statistical methodology), 71(2):319-392. Citado na pág. 19, 28, 65

Rue et al.(2017) Havard Rue, Andrea Riebler, Sigrunn H Sorbye, Janine B Illian, Daniel P Simpson e Finn K Lindgren. Bayesian computing with inla: a review. Annual Review of Statistics and Its Application, 4:395-421. Citado na pág. 65

Schervish(1995) Mark J Schervish. Theory of statistics. Springer Series in Statistics. Citado na pág. 3,8

Schnipke e Scrams(1999) Deborah L Schnipke e David J Scrams. Representing response-time information in item banks. law school admission council computerized testing report. lsac research report series. Citado na pág. 38

Schnipke e Scrams(2002) Deborah L Schnipke e David J Scrams. Exploring issues of examinee behavior: Insights gained from response-time analyses. Computer-based testing: Building the foundation for future assessments, páginas 237-266. Citado na pág. 37

Smithson e Shou(2017) Michael Smithson e Yiyun Shou. Cdf-quantile distributions for modelling random variables on the unit interval. British Journal of Mathematical and Statistical Psychology, 70(3):412-438. Citado na pág. 17

Song et al.(2004) Peter X-K Song, Zhenguo Qiu e Ming Tan. Modelling heterogeneous dispersion in marginal models for longitudinal proportional data. Biometrical Journal, 46(5):540-553. Citado na pág. 17

Song e Tan(2000) Peter Xue-Kun Song e Ming Tan. Marginal models for longitudinal continuous proportional data. Biometrics, 56(2):496-502. Citado na pág. 17

Spiegelhalter et al.(2003a) David Spiegelhalter, Andrew Thomas, Nicky Best e Dave Lunn. Winbugs user manual, 2003a. Citado na pág. 6

Spiegelhalter et al.(2003b) David Spiegelhalter, Andrew Thomas, Nicky Best e Dave Lunn. WinBUGS User Manual. MRC Biostatistics Unit, Cambridge, UK, 2003b. URL http://www. mrc-bsu.cam.ac.uk/bugs. Citado na pág. 80

Spiegelhalter et al.(2014) David Spiegelhalter, Andrew Thomas, Nicky Best e Dave Lunn. OpenBUGS Version 3.2.3 User Manual, Mar 2014. http://www.openbugs.net/Manuals/Manual.html. Citado na pág. 36

Spiegelhalter et al.(2002) David J Spiegelhalter, Nicola G Best, Bradley P Carlin e Angelika Van Der Linde. Bayesian measures of model complexity and fit. Journal of the Royal Statistical Society: Series B (Statistical Methodology), 64(4):583-639. Citado na pág. 28

Stan-Development-Team(2016) Stan-Development-Team. Stan Modeling Language User's Guide and Reference Manual, 2016. Version 2.12.0. Citado na pág. 6, 11

Tao et al.(2013) Jian Tao, Bao Xu, Ning-Zhong Shi e Hong Jiao. Refining the two-parameter testlet response model by introducing testlet discrimination parameters. Japanese Psychological Research, 55(3):284-291. Citado na pág. 6

Thaden e Kneib(2018) Hauke Thaden e Thomas Kneib. Structural equation models for dealing with spatial confounding. The American Statistician, 72(3):239-252. Citado na pág. 35

Thorndike(1917) Edward L Thorndike. Reading as reasoning: A study of mistakes in paragraph reading. Journal of Educational psychology, 8(6):323. Citado na pág. 1 
Unidad de Medición de la Calidad Educativa(2012) Unidad de Medición de la Calidad Educativa. Resultados de la Evaluación Censal de Estudiantes 2012. Ministerio de Educación, Lima, Perú, 2012. Citado na pág. 19

van der Linden(2006) Wim $\mathrm{J}$ van der Linden. A lognormal model for response times on test items. Journal of Educational and Behavioral Statistics, 31(2):181-204. Citado na pág. 43

van der Linden(2007) Wim J van der Linden. A hierarchical framework for modeling speed and accuracy on test items. Psychometrika, 72(3):287. Citado na pág. 1, 37, 38, 42, 43, 44, 54

van der Linden(2016) Wim $\mathrm{J}$ van der Linden. Handbook of item response theory. Taylor \& Francis Group. Citado na pág. 1, 7, 8

Vats et al.(2015) Dootika Vats, James M Flegal e Galin L Jones. Multivariate output analysis for markov chain monte carlo. arXiv preprint arXiv:1512.07713. Citado na pág. 12

Vehtari et al.(2017) Aki Vehtari, Andrew Gelman e Jonah Gabry. Practical bayesian model evaluation using leave-one-out cross-validation and waic. Statistics and computing, 27(5):14131432. Citado na pág. 13

Verkuilen e Smithson(2012) J. Verkuilen e M Smithson. Mixed and mixture regression models for continuous bounded responses using the beta distribution. Journal of Educational and Behavioral Statistics, 37:82-113. Citado na pág. 17

von Davier(2008) Matthias von Davier. A general diagnostic model applied to language testing data. British Journal of Mathematical and Statistical Psychology, 61(2):287-307. Citado na pág. 1

Wainer e Kiely(1987) Howard Wainer e Gerard L. Kiely. Item clusters and computarized adaptative testing: A case for testlets. Journal of Educational Measurement, 24 (3):185-201. Citado na pág. 5

Wainer et al.(2007a) Howard Wainer, Eric T. Bradlow e Xiaohui Wang. Testlet response theory and its application. Cambridge University Press. Citado na pág. 5, 6, 7

Wainer et al.(2007b) Howard Wainer, Eric T Bradlow e Xiaohui Wang. Testlet response theory and its applications. Cambridge University Press. Citado na pág. 6

Wang e Wilson(2005) Wen-Chung Wang e Mark Wilson. The rasch testlet model. Applied Psychological Measurement, 29 (2):126-149. Citado na pág. 5

Wang et al.(2002) Xiaohui Wang, Eric T Bradlow e Howard Wainer. A general bayesian model for testlets: Theory and applications. Applied Psychological Measurement, 26(1):109-128. Citado na pág. $1,5,9$

Wang et al.(2005) Xiaohui Wang, Eric T. Bradlow e Howard Wainer. User's Guide for SCORIGHT (Version 3.0): Computer Program for Scoring Tests Built of Testlets Including a Module for Covariate Analysis. Educational Testing Service, 2005. ETS Research Report. Citado na pág. 6

Watanabe(2009) Sumio Watanabe. Algebraic geometry and statistical learning theory. Cambridge Monographs on Applied and Computational Mathematics. CUP. ISBN 9780521864671,0521864674. URL http://gen.lib.rus.ec/book/index.php?md5= 2967FDAAB00856C4B5573A816F5A4F25. Citado na pág. 28

Zhan et al.(2018) Peida Zhan, Manqian Liao e Yufang Bian. Joint testlet cognitive diagnosis modeling for paired local item dependence in response times and response accuracy. Frontiers in Psychology, 9:607. Citado na pág. 1, 37, 43

Zhang et al.(2016) Peng Zhang, Zhenguo Qiu e Chengchun Shi. simplexreg: An r package for regression analysis of proportional data using the simplex distribution. Journal of Statistical Software, 71(1):1-21. Citado na pág. 